\title{
On the nature of the galactic early-B hypergiants ${ }^{\star}$
}

\author{
J. S. Clark ${ }^{1}$, F. Najarro ${ }^{2}$, I. Negueruela ${ }^{3}$, B. W. Ritchie ${ }^{1}$, M. A. Urbaneja ${ }^{4}$, and I. D. Howarth ${ }^{5}$ \\ 1 Department of Physics and Astronomy, The Open University, Walton Hall, Milton Keynes, MK7 6AA, UK \\ e-mail: s.clark@open.ac.uk \\ 2 Departamento de Astrofísica, Centro de Astrobiología, (CSIC-INTA), Ctra. Torrejón a Ajalvir, km 4, 28850 Torrejón de Ardoz, \\ Madrid, Spain \\ 3 Departamento. de Física, Ingeniería de Sistemas y Teoría de la Señal, Universidad de Alicante, Apdo. 99, 03080 Alicante, Spain \\ ${ }^{4}$ Institute for Astronomy, University of Hawaii, 2680 Woodlawn Drive, Honolulu, Hawaii 96822, USA \\ 5 Department of Physics \& Astronomy, University College London, Gower Street, London, WC1E 6BT, UK
}

Received 14 June 2011 / Accepted 27 January 2012

ABSTRACT

\begin{abstract}
Aims. Despite their importance to a number of astrophysical fields, the lifecycles of very massive stars are still poorly defined. In order to address this shortcoming, we present a detailed quantitative study of the physical properties of four early-B hypergiants (BHGs) of spectral type B1-4 Ia+ ; Cyg OB2 \#12, $\zeta^{1}$ Sco, HD 190603 and BP Cru. These are combined with an analysis of their long-term spectroscopic and photometric behaviour in order to determine their evolutionary status.

Methods. Quantitative analysis of UV-radio photometric and spectroscopic datasets was undertaken with a non-LTE model atmosphere code in order to derive physical parameters for comparison with apparently closely related objects, such as B supergiants (BSGs) and luminous blue variables (LBVs), and theoretical evolutionary predictions.

Results. The long-term photospheric and spectroscopic datasets compiled for the early-B HGs revealed that they are remarkably stable over long periods ( $\geq 40 \mathrm{yrs}$ ), with the possible exception of $\zeta^{1}$ Sco prior to the 20th century; in contrast to the typical excursions that characterise LBVs. Quantitative analysis of $\zeta^{1}$ Sco, HD 190603 and BP Cru yielded physical properties intermediate between BSGs and LBVs; we therefore suggest that BHGs are the immediate descendants and progenitors (respectively) of such stars, for initial masses in the range $\sim 30-60 M_{\odot}$. Comparison of the properties of $\zeta^{1}$ Sco with the stellar population of its host cluster/association NGC 6231/Sco OB1 provides further support for such an evolutionary scenario. In contrast, while the wind properties of Cyg OB2 \#12 are consistent with this hypothesis, the combination of extreme luminosity and spectroscopic mass $\left(\sim 110 M_{\odot}\right)$ and comparatively low temperature means it cannot be accommodated in such a scheme. Likewise, despite its co-location with several LBVs above the Humphreys-Davidson (HD) limit, the lack of long term variability and its unevolved chemistry apparently excludes such an identification. Since such massive stars are not expected to evolve to such cool temperatures, instead traversing an

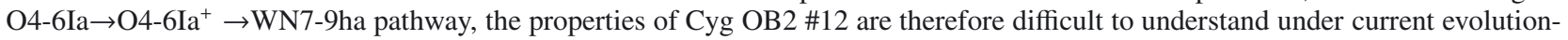
ary paradigms. Finally, we note that as with AG Car in its cool phase, despite exceeding the HD limit, the properties of Cyg OB2 \#12 imply that it lies below the Eddington limit - thus we conclude that the HD limit does not define a region of the HR diagram inherently inimical to the presence of massive stars.
\end{abstract}

Key words. stars: evolution - stars: early-type - supergiants - stars: fundamental parameters - stars: mass-loss

\section{Introduction}

Very massive stars are of considerable astrophysical interest given their role in driving galactic evolution via the copious production of ionising radiation and the deposition of chemically processed material and mechanical energy into the interstellar medium. They are thought to be the progenitors of Type Ibc and II supernovae ( $\mathrm{SNe}$ ) and, in low metallicity environments, gamma ray bursts (GRBs). The diverse nature of their core collapse $\mathrm{SNe}$ is also mirrored in the nature of their relativistic remnants which, for a given progenitor mass, may be either a neutron star or black hole depending on their pre-SNe (binary mediated?) mass loss history (e.g. Ritchie et al. 2010). Moreover, with $M_{V} \sim-11$ during particular phases of their postmain sequence evolution, they have the potential to probe the distance to - and chemical composition and star formation history of - external galaxies out to distances of $\sim 10$ to $30 \mathrm{Mpc}$

\footnotetext{
^ Appendices are available in electronic form at http://www . aanda.org
}

with the current and next generation of ground based telescopes (Kudritzki 2010).

Unfortunately, despite considerable theoretical efforts (e.g. Meynet \& Maeder 2005; Heger et al. 2000) we currently lack a comprehensive theoretical framework to fully understand and exploit all aspects of these stars; a problem compounded by comparatively weak observational constraints on a number of key physical processes that drive massive stellar evolution, such as the influence of (metallicity dependent) mass loss, the role of rotational mixing and the effect of binary interactions. In this regard, a critical test of evolutionary theory is the accurate reproduction of the properties of stars within the short lived transitional phase between the main sequence (MS) and H-depleted Wolf-Rayets (WRs), which is populated by a diverse "zoo" of disparate objects such as blue and yellow hypergiants (B/YHGs) and luminous blue variables (LBVs)/P-Cygni supergiants. For example, a crucial observational finding that must be replicated is the apparent dearth of cool evolved stars at high luminosities (the empirical Humphreys-Davidson (HD) limit; Humphreys \& Davidson 1979); in this regard the YHG/RSG populations of 
M 31 (Drout et al. 2009) and the Galactic cluster Westerlund 1 (Clark et al. 2010) already pose problems for current theoretical models.

Another manifestation of this uncertainty is an inability to incorporate the various members of the post-MS "zoo" into a coherent evolutionary scheme as a function of initial stellar mass. Various authors (e.g. Langer et al. 1994; Crowther et al. 1995; Martins et al. 2007, 2008) have proposed scenarios which have increasingly been informed by quantitative non-LTE model atmosphere analysis of different stellar populations, latterly located within coeval young massive Galactic clusters. In order to build on this approach we present an analysis of the properties of the BHGs of early B spectral type, which hitherto have escaped systematic study.

The Ia ${ }^{+}$luminosity class was first applied to a group of four highly luminous $\left(M_{V}<-8\right)$ B "super-supergiants" within the LMC by Keenan (1971), which were later described as "hypergiants" by van Genderen et al. (1982). These are further distinguished from normal BSGs by the presence of (P Cygni) emission in the Balmer series (cf. HD 190603; Lennon et al. 1992). Currently, to the best of our knowledge only 16 (candidate) BHGs have been identified within the Galaxy, of which eight are of early $(\leq \mathrm{B} 4)$ and eight of late $(\geq \mathrm{B} 5)$ spectral type; these are summarised in Table 1. Of the early BHGs, Kaper et al. (2006) have already presented the results of a quantitative analysis of BP Cru (=Wray 977; the mass donor in the High Mass X-ray Binary GX301-2); in this paper we present the result of equivalent analyses of Cyg OB2 \#12, $\zeta^{1}$ Sco(=HD 152236) and HD 190603. Foreshadowing Sect. 4, while the latter two objects have previously been subject to similar studies (e.g. Crowther et al. 2006; Searle et al. 2008), we employ more extensive multiwavelength datasets that enable significantly more accurate determinations of physical parameters; for example the lack of wind contamination in the higher Balmer and Paschen lines sampled here permitting a more robust determination of the surface gravity. Insufficient data exist to undertake comparable modeling of the remaining early B hypergiants, although we are currently in the process of obtaining the requisite observations.

Of these stars, Cyg OB2 \#12 is of particular interest as it has long been recognised as one of the intrinsically brightest, and potentially most luminous stars in the Galaxy (=Schulte 12; Sharpless 1957; Schulte 1958), lying well above the HD limit and so should provide a stringent test of current evolutionary theory. Moreover, BHGs have previously been associated with the LBV phenomenon by various authors (e.g. Clark et al. 2005a) and LBVs in turn have been implicated as both critical to the formation of WRs and as the immediate precursors of type II SNe (Smith \& Conti 2008). Finally, Cyg OB2 \#12 and $\zeta^{1}$ Sco are generally thought to be located within the Cyg OB2 and Sco OB1 associations respectively, which have both benefited from multiple (recent) studies ${ }^{1}$ and hence in principal should help in the assessment of both the nature of their progenitors as well as their placement in a post-MS evolutionary sequence.

The paper is structured as follows. In Sect. 2 we briefly detail the new observations of the 3 BHGs analysed in this work (Cyg OB2 \#12, $\zeta^{1}$ Sco and HD 190603; we utilise the dataset and analysis of Kaper et al. (2006) for BP Cru), while summarising the consolidated observational datasets in Sect. 3. This enables us to assess the degree of short and long-term variability

\footnotetext{
1 E.g. Cyg OB2: Massey \& Thompson (1991), Knödlseder (2000), Comerón et al. (2002), Hanson (2003) \& Negueruela et al. (2008) and Sco OB1: Reipurth (2008), Sana et al. (2006a,2007; 2008) and Raboud et al. (1997).
}

demonstrated by the program stars as well as providing the spectra and optical-radio spectral energy distributions (SEDs) for the non-LTE model atmosphere quantitative analysis. The results of this are presented in Sect. 4, discussed in Sect. 5 and conclusions presented in Sect. 6. Finally, an extensive summary of the (historical) observational properties of Galactic BHGs is documented in Appendix A, the spectropolarimety of Cyg OB2 \#12 and $\zeta^{1}$ Sco discussed in Sect. 4 is presented in Appendix B and Appendix $\mathrm{C}$ contains an analysis of the properties of the host cluster (NGC 6231) and association (Sco OB1) of $\zeta^{1}$ Sco.

\section{Observations and data reduction}

In order to accomplish the goals of the paper we have compiled extensive datasets for Cyg OB2 \#12, $\zeta^{1}$ Sco and HD 190603, utilising both new and published observations. A full presentation of the data for these (and other) BHGs may be found in Appendix A, while we briefly describe the new observations undertaken and data reduction employed below. In Appendix B we also present the results of analysis of previously unpublished spectropolarimetric observations of Cyg OB2 \#12 and $\zeta^{1}$ Sco; while these are not directly employed in the quantitative modeling of these stars they serve as a useful check on the geometry of the cirumstellar environment of these stars.

\subsection{Cyg OB2 \#12}

New high resolution and $\mathrm{S} / \mathrm{N}$ spectra of Cyg OB2 \#12 were obtained through the William Herschel Telescope (WHT) service programme on 2008 July 28, employing the ISIS doublebeam spectrograph equipped with the R1200B and R1200R gratings; a summary of the resultant wavelength ranges is given in Table 2. These observations were supplemented with a further five epochs of previous unpublished archival observations dating from 1992-2007; these too are summarised in Table 2. All spectra were reduced in a consistent manner using the Starlink packages Figaro and Kappa, with selected regions presented in Figs. 1, 2 and A.1. Continuum normalisation was accomplished via spline fitting and division, yielding uncertainties of a few percent at most. Likewise, the RMS on the fits to the arc spectra are of the order of $\sim 0.03$ pixels, resulting in a negligible error for wavelength callibration.

Unfortunately the distance and reddening to Cyg OB2 \#12 preclude UV observations, so the 4000-9000 A spectrum obtained in 2008 formed the primary dataset employed in this study. This was supplemented with the $1 \mu \mathrm{m}$ spectrum of Conti \& Howarth (1999), which encompasses the important He I $1.083 \mu \mathrm{m}$ transition, as well as the flux callibrated ISO-SWS spectrum presented by Whittet et al. (1997) and an archival Spitzer Space Telescope IRS spectrum (Houck et al. 2004). An optical - radio spectral energy distribution (SED) was constructed from continuum flux measurements from the literature (Tables A.1-A.3). While these observations were not contemporaneous, as demonstrated in Sect. A.1 there is no evidence for substantial variability/evolution over the timeframe spanned by these observations. Hence we are confident that such an approach is well justified and this is supported a posteriori by the excellent fits to the combined dataset.

\section{2. $\zeta^{1} S c O$}

A total of 18 new spectra have been utilised for the analysis of the long-term behaviour of $\zeta^{1}$ Sco. Unpublished 

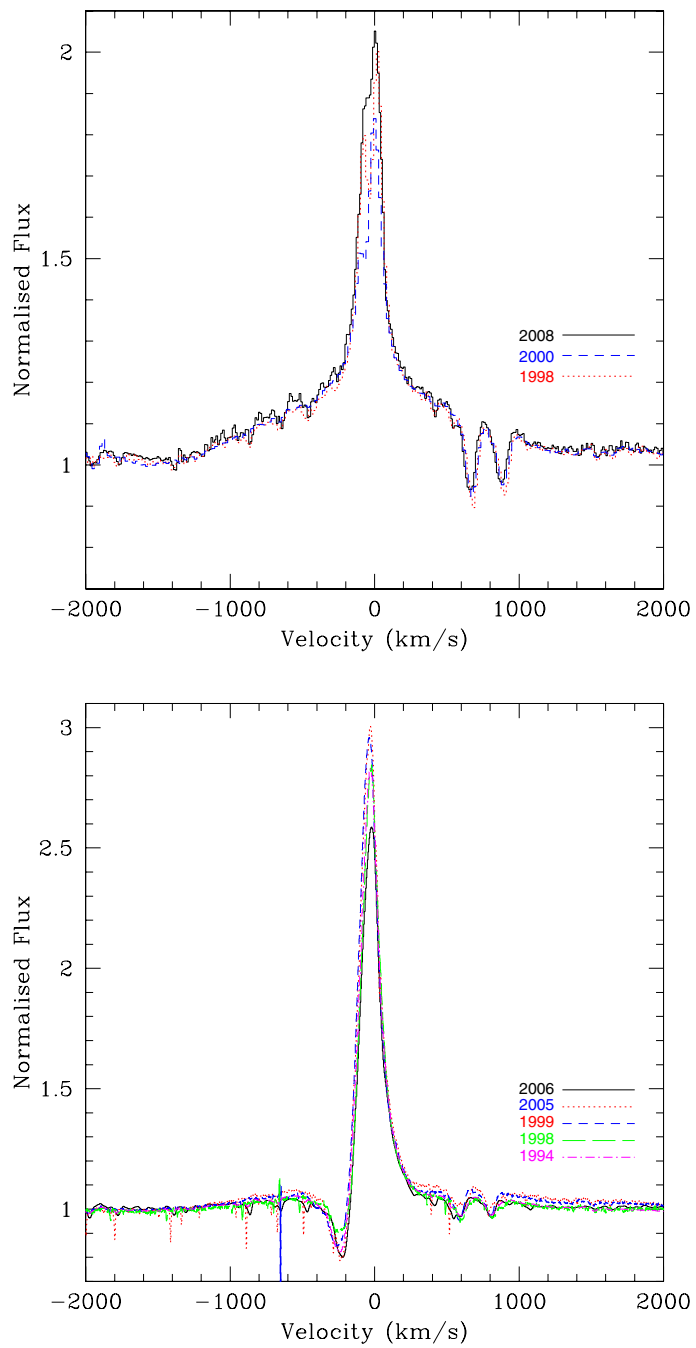

Fig. 1. Comparison of three (1999-2008) and five (1994-2009) epochs of $\mathrm{H} \alpha$ observations for Cyg OB2 \#12 (upper panel) and $\zeta^{1}$ Sco (lower panel) demonstrating line profile variability at low projected velocities. Note the corresponding lack of variability in the line wings and the С пा $\lambda \lambda$ 6578, 6582 doublet in the red wing. Due to their lower resolution, spectra of Cyg OB2 \#12 from 1992+5 are not presented, but are consistent to these spectra when all are convolved to a matching resolution.

ESO $2.2 \mathrm{~m} /$ FEROS spectra from 1998-9 (Program ID 063.H0080; PI: Kaufer) and 2005 (Program ID 075.D-0103(A); PI:Dufton) were kindly provided by Otmar Stahl (2010, priv. comm.) and Phillip Dufton (2010, priv. comm.) respectively. Data reduction was accomplished via the custom pipeline described in Stahl et al. (1999). Continuum normalisation was accomplished by first dividing the spectra by the instrumental response curve, with a subsequent division by a spline function; as before, the errors in this procedure are of the order of a few percent. The spectrum from 2006 February 17 was obtained by us with the ESO NTT/EMMI; standard reduction procedures were employed and are discussed in Negueruela et al. (in prep.). Finally new blue and red end spectra from 2009 March 06 were obtained from the ESO archive (Program ID 082.C-0566(A); PI: Beletsky). These were obtained with the VLT/UVES with cross disperser gratings CD1, 2 and 3 and were reduced using the standard ESO pipeline. A full summary of these new observations is provided in Table 2 and selected spectra are presented in Figs. 1, 2 and A.1. The full dataset used for modeling also in-
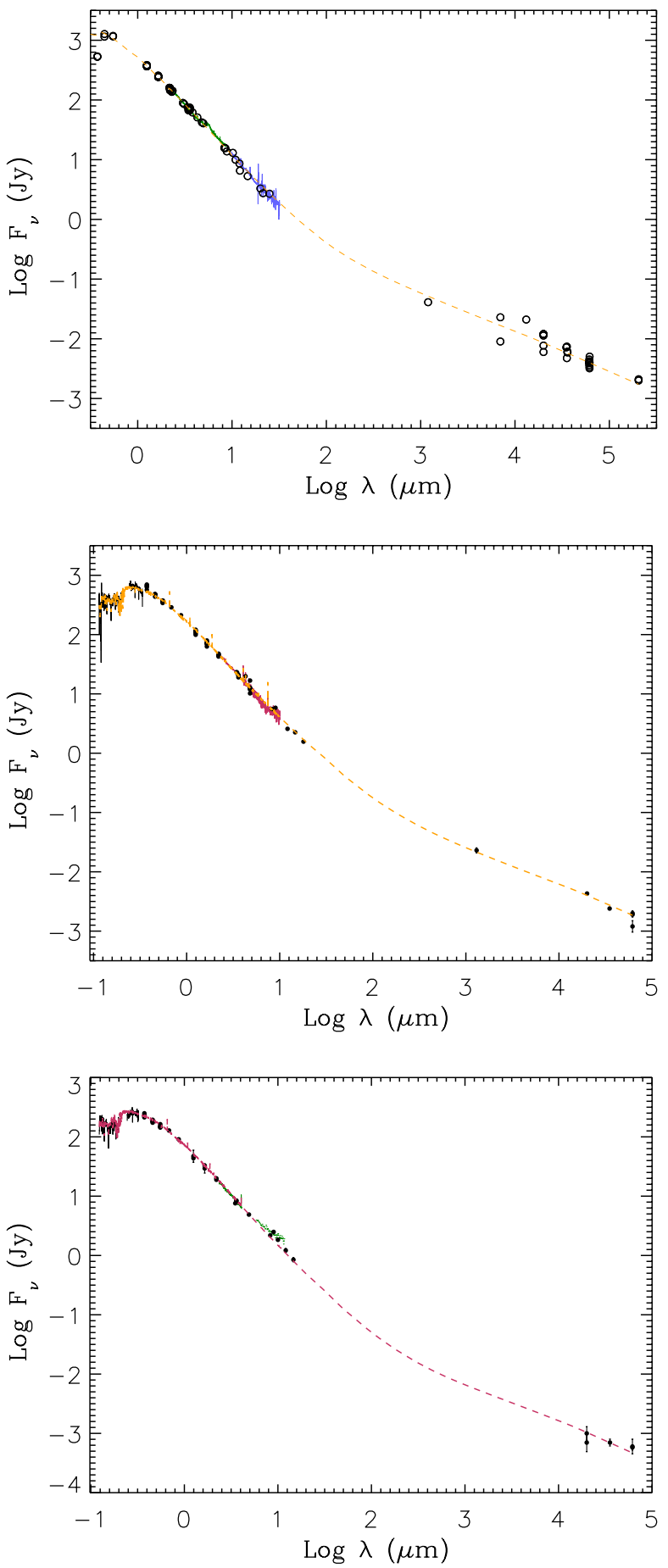

Fig. 2. Comparison of synthetic spectra (yellow dashed line) with the optical to radio SEDs of Cyg OB2 \#12 (top panel), $\zeta^{1}$ Sco (middle panel) and HD 190603 (bottom panel). Note the lack of a near-mid IR excess signalling the absence of significant amounts (warm) circumstellar dust. Physical parameters employed in the modeling - such as $L_{\text {bol }}$ and $E(B-V)-$ may be found in Table 4 and Sects. 4.1 and 4.2.

cludes archival IUE and ISO spectra, as well as published photometry summarised in Tables A.1-A.3.

\section{3. $H D 190603$}

Finally, we make use of solely archival data for the quantitative analysis of HD 190603. As with the other objects, the sources of data used in constructing the SED are given in Tables A.1-A.3. Spectroscopic data were taken from Lennon 
et al. (3950-5000 ̊+H $\alpha$; 1992), Rivinius et al. (4050-6800 ; 1997), Andrillat et al. (8390-8770 $\AA$ ) as well as one from the IACOB database (3710-6800 Å; Sergio Simón-Díaz 2011, priv. comm.).

\section{The observational properties of BHGs}

BHGs form a spectroscopically homogeneous class of objects that are distinguished from BSGs by the presence of Balmer emission lines. However, they have been implicated in the LBV phenomenon (Sect. 1) and so it is of interest to compare their 0.4-4.1 $\mu \mathrm{m}$ spectra with those of bona fide LBVs in both hot and cool phases. Suitable comparison spectra across this wavelength range are provided by, amongst others, Stahl et al. (2001), Hillier et al. (1998), Groh et al. (2007), Clark et al. (2003a, 2009, 2011) and Lenorzer et al. (2002). Significant differences are evident; specifically the weakness of the $\mathrm{H}_{\mathrm{I}}$ and $\mathrm{He}_{\mathrm{I}}$ lines in the BHG spectra, as well as the absence of emission in low excitation metallic lines such as Fe II and III that characterise the spectra of LBVs.

A second observational characteristic of some LBVs is the presence of circumstellar dust - indeed the presence of a detached circumstellar nebula has been advanced to support the LBV nature of the late-B hypergiant HD 168625. Of the early BHGs in question, only BP Cru is known to show evidence for warm dust (e.g. Fig. 2; Moon et al. 2007), although with $M_{\text {dust }} \sim 5.2 \times 10^{-8} M_{\odot}$, considerably less than is associated with either LBVs or supergiant B[e] stars (Egan et al. 2002; Clark et al. 2003a; Kastner et al. 2006).

However, the defining characteristic of LBVs is their dramatic spectroscopic and photometric variability over timescales of months-years. Motivated by reports of spectral variability amongst both early- and late-B HGs we undertook an exhaustive literature search for these objects, which we present in Appendix A, and briefly discuss here. As highlighted by previous authors, there is strong observational evidence for a physical association between LBVs and low-luminosity, late-B HGs such as HD 160529 and HD 168607; however, the link is far from proven for earlier, more luminous BHGs. Owing to their luminosities extensive spectroscopic and photometric datasets exist for such stars, with the majority extending back to the mid-20th century, for Cyg OB2 \#12 and HD 190603 to the turn of last century and (sparse) photometric data for $\zeta^{1}$ Sco potentially many centuries before that (Appendix A) ${ }^{2}$.

Cyg OB2 \#12 has long been suspected of being spectroscopically variable, being variously classified as B3-8 $\mathrm{Ia}^{+}$in the literature (e.g. Table A.4 and refs. therein). However, upon close examination of the spectra and classification criteria employed, we conclude that long-term evolution of the stellar temperature between 1954-2008 is probably absent (Appendix A.1.2) and that the reports of such behaviour result from comparison of low $\mathrm{S} / \mathrm{N}$ and resolution spectra and, crucially, the sensitivity of the commonly employed He I $4471 / \mathrm{Mg}$ II 4481 criterion to the properties of the photosphere/wind transition zone as well as photospheric temperature (Sect. 4.1). This conclusion is bolstered by the lack of secular photospheric variability dating back to the 1890s, although low level, apparently aperiodic variability does appear present (e.g. Gottlieb \& Liller 1978).

\footnotetext{
2 Unfortunately, in many cases early observations are presented in the literature without precise observational dates. Nevertheless, the similarity of these data to more recent observations supports the lack of long term variability in such cases.
}
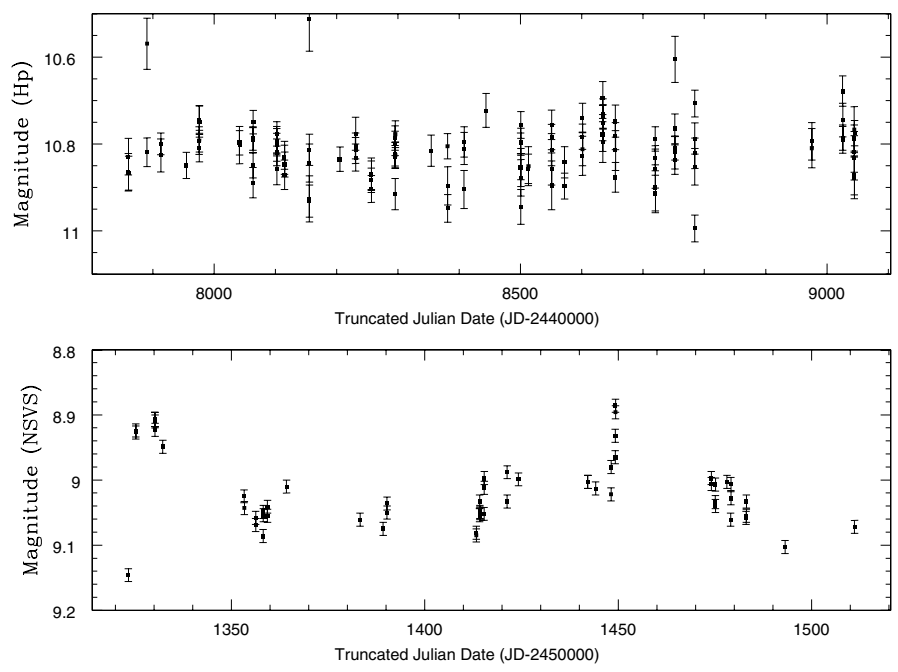

Fig. 3. Hipparcos (top panel: 1989-1993) and NSVS (bottom panel: 1999-2000) lightcurves of Cyg OB2 \#12. Both panels plot the (non standard) instrumental magnitudes (references to these are provided in the text) and we caution that the scale of both axes differ between the two panels.

Based on the datasets discussed in Appendix A, similar conclusions may be drawn for the remaining early- (BP Cru, HD 80077, HD 190603, HD 168454, Wd1-5 and 13) and mid-B HGs (Wd1-7 and 33), with the potential exception of $\zeta^{1}$ Sco. Spectroscopically, it is the best sampled of all the BHGs and shows no evidence for evolution between $\sim 1891-2009$ (Table A.5). Likewise, it appears to have remained photometrically stable since at least 1949 (Table A.1), although isolated historical photometric observations dating back many centuries are available in the literature, from which Sterken et al. (1997) infer possible LBV like variability in the 18-19th century.

However, while long-term variability appears rare or absent amongst the early-B HGs, rapid ( day to day) line profile variability (LPV) in both wind dominated emission and photospheric absorption lines appears ubiquitous, persistent and well documented (e.g. Fig. 1 and Appendix A and references therein). These behaviours are thought to arise from time variable wind structure and photospheric pulsations respectively, with the former also potentially giving rise to variable radio continuum emission (e.g. González \& Cantó 2008), although changes in the the degree of ionization of the wind are another possible cause of this phenomenon.

Likewise, rapid, low amplitude photometric variability is present amongst all the BHGs (e.g. Fig. 3 and Appendix A). Such behaviour has long been recognised as being characteristic of luminous stars of all spectral types (the " $\alpha$ Cygni" variables; e.g. Burki 1978; van Leeuwen et al. 1998; Clark et al. 2010) and again is typically attributed to photospheric pulsations.

We conclude that the temporal behaviour of the early-B HGs appears entirely typical of the wider population of luminous, early-spectral-type non-LBV stars, with the potential exception of $\zeta^{1}$ Sco in the 18-19th centuries and a possible sudden $\Delta m_{\mathrm{B}} \sim 0.4 \mathrm{mag}$ "glitch" in the lightcurve of Cyg OB2 \#12 in the mid-1940s (Gottlieb \& Liller 1978).

\section{Quantitative modeling}

In order to more fully address the physical properties of Cyg OB2 \#12, $\zeta^{1}$ Sco and HD 190603 we have employed the 
Table 1. Summary of Galactic BHG properties.

\begin{tabular}{|c|c|c|c|c|c|}
\hline \multirow[t]{2}{*}{ Star } & \multirow{2}{*}{$\begin{array}{c}\text { Spectral } \\
\text { type }\end{array}$} & \multicolumn{2}{|c|}{ Co-ordinates } & \multirow{2}{*}{$\begin{array}{l}V \text { band } \\
\text { (mean) }\end{array}$} & \multirow{2}{*}{$\begin{array}{c}\text { Cluster/ } \\
\text { association }\end{array}$} \\
\hline & & RA & Dec. & & \\
\hline BP Cru & $\mathrm{B} 1 \mathrm{Ia}^{+}$ & 122637.56 & -624613.2 & 10.68 & - \\
\hline HD 169454 & $\mathrm{~B} 1 \mathrm{Ia}^{+}$ & 182515.19 & -135842.3 & 6.65 & - \\
\hline$\zeta^{1} \mathrm{Sco}$ & B $1.5 \mathrm{Ia}^{+}$ & 165349.73 & -422143.3 & 4.78 & Sco OB1 \\
\hline HD 190603 & B $1.5 \mathrm{Ia}^{+}$ & 200436.17 & +321307.0 & 5.66 & - \\
\hline HD 80077 & $\mathrm{~B} 2.5 \mathrm{Ia}^{+}$ & 091554.79 & -495824.6 & 7.57 & Pismis 11 \\
\hline Cyg OB2 \#12 & B3-4 $\mathrm{Ia}^{+}$ & 203240.96 & +411429.3 & 11.47 & Cyg OB2 \\
\hline Wd1-5 & $\mathrm{WNL} / \mathrm{B} \mathrm{Ia}^{+}$ & 164602.97 & -455019.5 & 17.49 & Wd1 \\
\hline Wd1-13 & $\mathrm{WNL} / \mathrm{B} \mathrm{Ia}^{+}$ & 164706.45 & -455026.0 & 17.19 & Wd1 \\
\hline Wd1-7 & $\mathrm{B} 5 \mathrm{Ia}^{+}$ & 164603.62 & -455014.2 & 15.57 & Wd1 \\
\hline Wd1-33 & $\mathrm{B} 5 \mathrm{Ia}^{+}$ & 164704.12 & -455048.3 & 15.61 & Wd1 \\
\hline HD 183143 & B7 Iae & 192726.56 & +181745.2 & 6.92 & - \\
\hline HD 199478 & B9 Iae & 205549.80 & +472503.6 & 5.73 & - \\
\hline HD 168625 & $\mathrm{~B} 8 \mathrm{Ia}^{+}$ & 182119.55 & -162226.1 & 8.44 & - \\
\hline Wd1-42a & B9 $\mathrm{Ia}^{+}$ & 164703.25 & -455052.1 & - & Wd1 \\
\hline HD 168607 & B9 $\mathrm{Ia}^{+}$ & 182114.89 & -162231.8 & - & - \\
\hline HD 160529 & B8-A9 $\mathrm{Ia}^{+}$ & 174159.03 & -333013.7 & - & - \\
\hline
\end{tabular}

Notes. No V-band magnitude is available for Wd1-42a, while both HD 168607 and 160529 are known large amplitude photometric variables (Sect. A.6).

Table 2. Summary of unpublished spectral observations.

\begin{tabular}{|c|c|c|c|}
\hline Date & Telescope & $\begin{array}{l}\text { Wavelength } \\
\text { range }(\AA)\end{array}$ & Resln. \\
\hline & Cyg\#12 & & \\
\hline \multirow[t]{2}{*}{1992 July 17} & INT & $4036-4836$ & 4000 \\
\hline & & $5765-9586^{a}$ & 4000 \\
\hline 1995 July 17 & WHT & $6172-6980$ & 4000 \\
\hline \multirow{2}{*}{1998 Aug. 09} & WHT & $3940-5737$ & 8000 \\
\hline & & $6366-6772$ & 8000 \\
\hline \multirow[t]{2}{*}{2000 July 17} & INT & $3940-5737$ & 8000 \\
\hline & & $6327-6727$ & 8000 \\
\hline 2007 Aug. 22 & WHT & $7600-8900$ & 10000 \\
\hline \multirow[t]{4}{*}{2008 July 22} & WHT & $4000-4700$ & 4500 \\
\hline & & $4500-5270$ & 4500 \\
\hline & & $6450-7150$ & 7000 \\
\hline & & $8350-8900$ & 10000 \\
\hline \multirow[b]{2}{*}{1998 Octoher 7} & $\zeta^{1}$ Sco & & \\
\hline & $\mathrm{ESO} 2.2-\mathrm{m}$ & $4000-8950^{b}$ & 48000 \\
\hline 1999 July-Aug. & ESO $2.2-\mathrm{m}^{c}$ & $4000-8950^{b}$ & 48000 \\
\hline 2005 April 24 & ESO $2.2 \mathrm{~m}$ & $3800-6800$ & 48000 \\
\hline 2006 February 17 & NTT & $3933-7985$ & 9840 \\
\hline 2009 March 06 & VLT & $3060-5600$ & \\
\hline
\end{tabular}

Notes. ${ }^{(a)}$ This wavelength coverage was obtained via 5 overlapping observations. ${ }^{(b)}$ Approximate usable wavelengths. ${ }^{(c)} 14$ spectra obtained between July 17 to August 4.

non-LTE model atmosphere code CMFGEN (Hillier \& Miller 1998, 1999). This solves the radiative-transfer equation for a spherically symmetric wind in the co-moving frame under the constraints of radiative and statistical equilibrium. Since the code does not solve for the wind structure from first (physical) principles, a velocity structure must be chosen; we adopted a standard $\beta$-type law ${ }^{3}$. Given the relatively high wind densities of BHGs, the role of the hydrostatic structure and the transition region between photosphere and wind becomes crucial to

\footnotetext{
3 The $\beta$-type law is given by $v(r)=v_{\infty} \times(1-b / r)^{\beta}$, where $b=r_{\text {phot }} \times$ $\left(1-\left(v_{\text {phot }} / v_{\infty}\right)^{(1 / \beta)}\right)$ and $r_{\text {phot }}$ and $v_{\text {phot }}$ are the radius and velocity at the connection point between the $\beta$-law and the hydrostatic structure.
}

interpret the spectra (e.g. Sect. 4.1 and Fig. 8). Because of this, we used a hydrostatic density structure at depth (the "pseudophotosphere") matched to a $\beta$-law wind, requiring the density to be continuous at the transition. The location of the transition is fixed by the adopted base velocity of the wind, $v_{\text {phot }}$ (a free parameter in the fitting), which specifies the density through the equation of mass continuity. This approach is similar to that of, e.g., Santolaya-Rey et al. (1997), except that in our implementation the flux-weighted mean opacity is used in preference to the Rosseland mean opacity Several comparisons using "exact" photospheric structures from TLUSTY (Hubeny \& Lanz 1995) showed excellent agreement with our method ${ }^{4}$.

The main atomic processes and data sets are discussed in Hillier \& Miller (1998, see Dessart \& Hillier 2010 for an updated description of the atomic data). The list of model atoms utilized in our calculations is provided in Table 3 , including for each ion the number of super and full levels and number of bound-bound transitions. The CMFGEN model is then prescribed by the stellar radius, $R_{*}$, the stellar luminosity, $L_{*}$, the mass-loss rate, $\dot{M}$, the velocity field, $v(r)$ (defined by $\beta, v_{\text {phot }}$ and the terminal velocity, $v_{\infty}$ ), the volume filling factor, $f$, characterizing the clumping of the stellar wind, and elemental abundances. The interstellar reddening parameters $E(B-V)$ and $R_{V}$ are also obtained by fitting the model SEDs to the available photometric data. Finally, the equatorial rotation velocity, $v_{\mathrm{e}} \sin i$, and the atmospheric macroturbulent velocity, $v_{\text {macro }}$, were estimated in Fourier space (Simón-Díaz \& Herrero 2007) from a selected sample of photospheric lines.

Given the number of free parameters required to specify a fit it is not possible to survey the full parameter space systematically in order to establish robust error estimates; hence errors quoted in this paper represent the range of values for which an acceptable fit to the data may be obtained. Nevertheless, we discuss error estimates for key parameters for each of the stars analysed below. The validity of this technique has been demonstrated by calibration to stars of similar temperature and luminosity for

\footnotetext{
${ }^{4}$ The latest version of CMFGEN, which allows the user to compute the exact hydrostatic structure, showed full consistency with our method. At this stage, we prefer our approach as it allows for more flexibility to study the crucial transition region.
} 
Table 3. Model atoms included in our calculations.

\begin{tabular}{lccc}
\hline \hline Species & \# Full levels & \# Super levels & \# Of transitions \\
\hline $\mathrm{H}_{\text {I }}$ & 30 & 30 & 435 \\
$\mathrm{He}_{\text {I }}$ & 59 & 59 & 592 \\
$\mathrm{He}_{\text {II }}$ & 5 & 5 & 10 \\
$\mathrm{C}_{\text {II }}$ & 80 & 45 & 648 \\
$\mathrm{C}_{\text {III }}$ & 38 & 22 & 149 \\
$\mathrm{C}_{\text {IV }}$ & 8 & 5 & 22 \\
$\mathrm{~N}_{\text {I }}$ & 63 & 25 & 344 \\
$\mathrm{~N}_{\text {II }}$ & 179 & 86 & 1936 \\
$\mathrm{~N}_{\text {III }}$ & 20 & 11 & 61 \\
$\mathrm{O}_{\text {I }}$ & 75 & 23 & 450 \\
$\mathrm{O}_{\text {II }}$ & 102 & 46 & 992 \\
$\mathrm{Na}_{\text {I }}$ & 44 & 18 & 345 \\
$\mathrm{Mg}_{\text {II }}$ & 50 & 37 & 597 \\
$\mathrm{Al}_{\text {II }}$ & 20 & 12 & 41 \\
$\mathrm{Al}_{\text {III }}$ & 45 & 17 & 362 \\
$\mathrm{Si}_{\text {II }}$ & 72 & 35 & 491 \\
$\mathrm{Si}_{\text {III }}$ & 83 & 47 & 532 \\
$\mathrm{Si}_{\text {IV }}$ & 28 & 17 & 129 \\
$\mathrm{~S}_{\text {II }}$ & 85 & 26 & 745 \\
$\mathrm{~S}_{\text {III }}$ & 63 & 31 & 331 \\
$\mathrm{Ca}_{\text {II }}$ & 32 & 25 & 157 \\
$\mathrm{Cr}_{\text {III }}$ & 209 & 36 & 2444 \\
$\mathrm{Cr}_{\text {IV }}$ & 242 & 30 & 4732 \\
$\mathrm{Fe}_{\text {II }}$ & 709 & 233 & 18011 \\
$\mathrm{Fe}_{\text {III }}$ & 812 & 87 & 21383 \\
$\mathrm{Fe}_{\text {IV }}$ & 417 & 62 & 8353 \\
$\mathrm{Ni}_{\text {II }}$ & 254 & 33 & 3354 \\
$\mathrm{Ni}_{\text {III }}$ & 336 & 34 & 5181 \\
$\mathrm{Ni}_{\text {IV }}$ & 294 & 56 & 4174 \\
\hline Total & 4160 & 1137 & 72827 \\
\hline & & &
\end{tabular}

Notes. ${ }^{(*)}$ Not used in Cyg OB2 \#12.

which UV, optical and near-IR data were available (Najarro et al. 1999; Najarro 2001).

A comparison of the predicted SEDs to our data for the three stars is presented in Fig. 2, while selected regions of the synthetic spectra are overplotted on the observed 4090-11050 $\AA$ spectrum of Cyg OB2 \#12 in Fig. 4, the UV-near IR ( 1200-22100 $\AA)$ spectrum of $\zeta^{1}$ Sco in Figs. 5 and 6 and the optical ( 4000-7000 $\AA$ ) spectrum of HD 190603 in Fig. 7. The optical data shown in Fig. 6 correspond to the 2006 ESO EMMI/NTT run. Fits to the earlier FEROS spectra supplied by Otmar Stahl - which provide a more extended wavelength coverage - are shown in Fig. D.1. The latter were used to obtain the final stellar properties as they encompass the high Balmer and Paschen lines which constrain the surface gravity. A summary of the results of the analyses for the 3 stars is presented in Table 4, along with the parameters of the B1 $\mathrm{Ia}^{+}$star BP Cru, obtained via an identical methodology by Kaper et al. (2006).

\subsection{Cyg OB2 \#12}

For the purposes of modeling Cyg OB2 \#12 we employed the dataset described in Sect. 3 and Appendix A. We have also assumed membership of Cyg OB2 and hence a distance, $d \sim$ $1.75 \mathrm{kpc}$ (e.g. Negueruela et al. 2008 and refs. therein); we return to this issue below.

Temperature, gravity and luminosity The available optical and NIR spectra allowed us to make use of several ionization equilibria to estimate the effective temperature, and thus constrain the ionization structure of Cyg OB2 \#12. Hence, we were able to utilize simultaneously $\mathrm{Si}$ III/Si II, O II/O I, N II/N I and $\mathrm{Fe}$ III/Fe II line ratios. The $\mathrm{S}_{\text {III } / \mathrm{S}}$ II equilibrium was only used for a consistency check, as the weak diagnostic S III $4253 \AA$ line suffers from a poorer $\mathrm{S} / \mathrm{N}$ ratio in the blue spectral region due to reddening. We refer the reader to Sect. A.1.2 for a detailed description of the available diagnostic lines.

When analyzing the ionization equilibria, we found that while lines belonging to higher ionization stages react sensitively to effective temperature and to lesser extent to gravity, those corresponding to the lower ionization stage display a high sensitivity not only to temperature and gravity, but also the location of the transition region between photosphere and wind, characterised by $v_{\text {phot }}$ (especially the Si II, Fe II and O I lines). This sensitivity is clearly illustrated in Fig. 8 (right panel), where the choice of $v_{\text {phot }}$ significantly affects the resulting strength of the Fe II $5170 \AA$ line, leaving the Fe III lines basically unaffected. Thus, if a lower transition velocity is chosen, a lower effective temperature is required to match the $\mathrm{Fe}$ III/ $\mathrm{Fe}$ II ratio. As we will show later, this effect is responsible for our somewhat lower $T_{\text {eff }}$ values when compared to other studies (see Sect. 4.2). Interestingly, Fig. 8 (left panel) also shows how the ratio of the diagnostic lines He I 4471 and $\mathrm{Mg}$ II $4481 \AA$, which are used for spectral typing, also shows a moderate dependence on the transition region. Thus, a model with $v_{\text {phot }}=0.5 v_{\text {sound }}$ will yield equally strong $\mathrm{He}_{\mathrm{I}} 4471$ and $\mathrm{Mg}_{\text {II }} 4481 \AA$, while if the transition takes place at $v_{\text {phot }}=0.05 v_{\text {sound }}$, He I $4471 \AA$ clearly becomes stronger than $\mathrm{Mg}$ II $4481 \AA$.

We note that this moderate dependence on the transition region becomes important in BHGs, due to the presence of a strong, dense wind and should be negligible in B supergiants, where the absorption lines will form in deeper photospheric layers that are largely unaffected by the photosphere-wind transition region. Therefore, despite the large number of diagnostics, our final estimate of the effective temperature, $\left(T_{\mathrm{eff}}=13.7 \mathrm{kK}\right.$, see Table 4) is subject to a moderate uncertainty. We find $+800 \mathrm{~K}$ and $-500 \mathrm{~K}$ as upper and lower error bounds.

Once the effective temperature was obtained, and assuming a distance of $d \sim 1.75 \mathrm{kpc}$, we proceeded to fit the observed SED of Cyg OB2 \#12 from the optical through radio (see Fig. 2) and hence derived the reddening, stellar radius and, therefore, the stellar luminosity. We found $E(B-V)=3.84$ and a reddening parameter $R_{V}=2.65$, corresponding to $A_{V}=10.18$. This value shows excellent agreement with the $A_{V}=10.20$ found by Torres et al. 1991, although they obtained a higher total to selective extinction parameter $\left(R_{V}=3.04\right)$

From these values we obtained a stellar radius of $246 R_{\odot}$ and a final luminosity of $1.9 \times 10^{6} L_{\odot}$. The temperature and resultant luminosity are broadly comparable to previous qualitative studies (e.g. Massey \& Thompson 1991; Hanson 2003), confirming that it is an extraordinarily luminous, but comparatively cool BHG.

The Paschen lines in the $I$ Band provide the best constraints for the surface gravity, especially the run of the line overlap among the higher members (Fig. 4). Compared to the classical optical diagnostic lines - $\mathrm{H} \gamma$ and $\mathrm{H} \delta$ - the higher Paschen series lines are significantly less affected by the stellar wind (and consequently clumping) and the wind/photosphere transition region. Nevertheless, the influence of the latter once again translates into a larger uncertainty in the (lower) error. Thus, we find $\log g=1.70_{-0.15}^{+0.08}$, corresponding to a spectroscopic mass of $M=110_{-31}^{+23} M_{\odot}$. 
J. S. Clark et al.: On the nature of the galactic early-B hypergiants
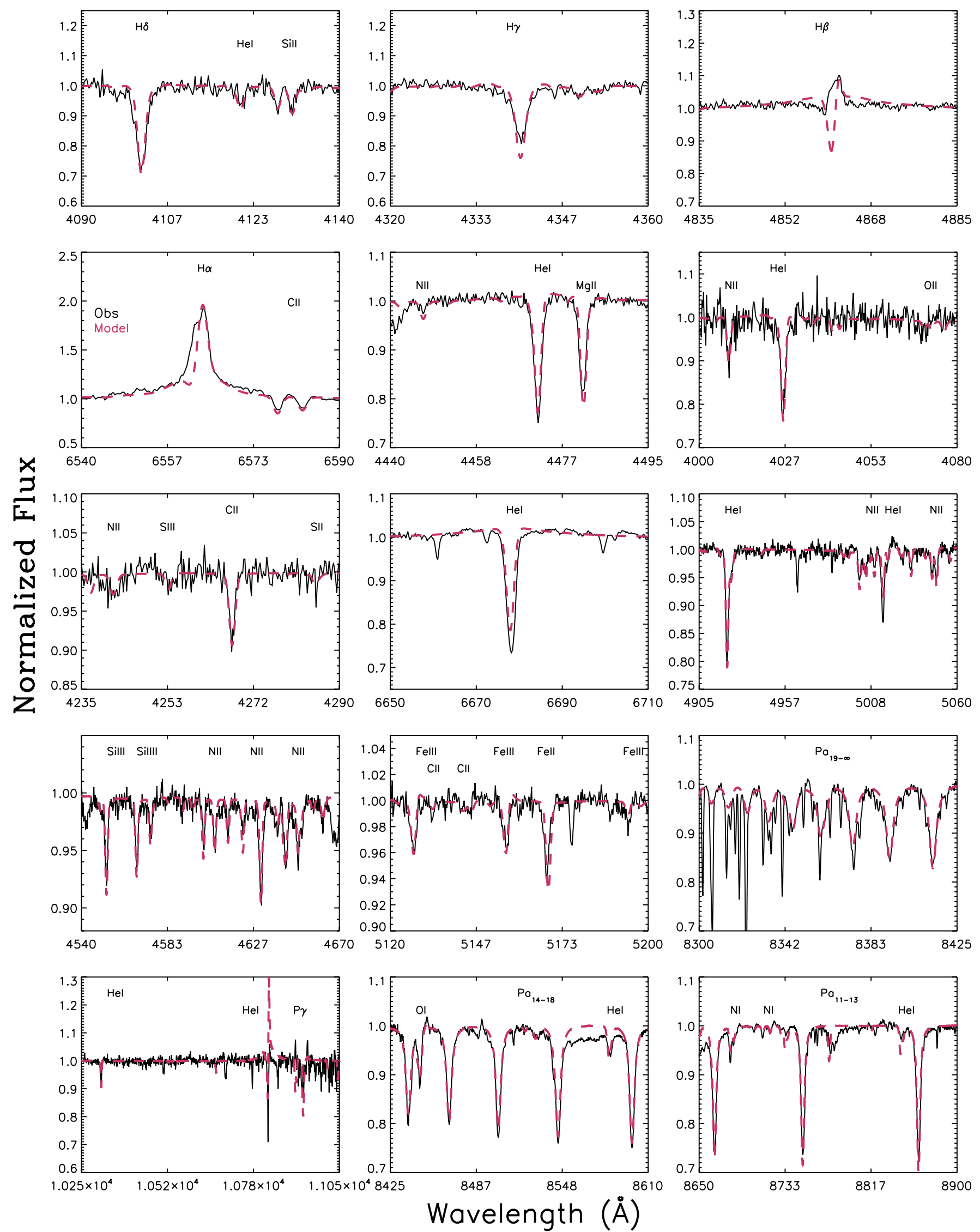

Fig. 4. Comparison of the synthetic spectrum of Cyg OB2 \#12 (red dashed line) with observational data for various transitions between 4090-11 $050 \AA$. Note that the narrow absorption lines which have not been labeled are telluric in origin. 
Table 4. Model parameters for the BHGs Cyg OB2 \#12, $\zeta^{1}$ Sco, HD 190603 \& BP Cru (upper panel) and Galactic B supergiant comparison (lower panel; Searle et al. 2008).

\begin{tabular}{|c|c|c|c|c|c|c|c|c|c|c|c|c|}
\hline Star & $\begin{array}{l}\text { Spec. } \\
\text { type }\end{array}$ & $\mathrm{DM}$ & $M_{V}$ & $\begin{array}{c}\log \left(L_{*}\right) \\
L_{\odot}\end{array}$ & $\begin{array}{l}R_{*} \\
R_{\odot}\end{array}$ & $\begin{array}{l}T_{\text {eff }} \\
\mathrm{kK}\end{array}$ & $\begin{array}{c}\dot{M} \\
10^{-6} M_{\odot} \mathrm{yr}^{-1}\end{array}$ & $\begin{array}{c}v_{\infty} \\
\mathrm{km} \mathrm{s}^{-1}\end{array}$ & $\beta$ & $f$ & $\log g$ & $\begin{array}{l}M_{*} \\
M_{\odot}\end{array}$ \\
\hline Cyg OB \#12 & B3-4 & 11.21 & -9.85 & 6.28 & 246.0 & 13.7 & 3.0 & 400 & 3.0 & 0.04 & 1.70 & 110 \\
\hline \multirow[t]{2}{*}{$\zeta^{1} \mathrm{Sco}$} & B1.5 & 11.07 & -8.50 & 5.93 & 103.0 & 17.2 & 1.55 & 390 & 2.25 & 0.06 & 1.97 & 36 \\
\hline & & 11.50 & -8.93 & 6.10 & 125.5 & 17.2 & 2.07 & 390 & & & & 53 \\
\hline \multirow[t]{2}{*}{ HD 190603} & B1.5 & 10.98 & -7.53 & 5.58 & 63.0 & 18.0 & 1.09 & 485 & 2.25 & 0.25 & 2.10 & 18 \\
\hline & & 12.38 & -8.93 & 6.14 & 120.0 & 18.0 & 2.87 & 485 & & & & 65 \\
\hline \multirow[t]{5}{*}{ BP Cru } & B1 & 12.41 & -7.47 & 5.67 & 70.0 & 18.1 & 10.0 & 305 & 2.25 & 1.0 & 2.38 & 43 \\
\hline & B1 & & & 5.44 & 36.5 & 22.0 & & & & & 2.41 & 12 \\
\hline & B1.5 & & & 5.44 & 44.5 & 19.9 & & & & & 2.41 & 18 \\
\hline & B3 & & & 5.37 & 60.4 & 16.4 & & & & & 2.16 & 19 \\
\hline & B4 & & & 5.34 & 63.5 & 15.8 & & & & & 2.06 & 16 \\
\hline
\end{tabular}

\begin{tabular}{lccccccccc}
\hline \hline Star & $\begin{array}{c}\text { Spec } \\
\text { type }\end{array}$ & $\begin{array}{c}v_{\text {turb }} \\
\mathrm{km} \mathrm{s}^{-1}\end{array}$ & $\begin{array}{c}v_{\mathrm{e}} \sin i \\
\mathrm{~km} \mathrm{~s}^{-1}\end{array}$ & $\begin{array}{c}v_{\text {macro }} \\
\mathrm{km} \mathrm{s}^{-1}\end{array}$ & $\begin{array}{c}v_{\text {phot }} \\
v_{\text {sound }}\end{array}$ & $\mathrm{H} / \mathrm{He}$ & $\mathrm{N} / \mathrm{N}_{\odot}$ & $\mathrm{C} / \mathrm{C}_{\odot}$ & $\mathrm{O} / \mathrm{O}_{\odot}$ \\
\hline Cyg OB \#12 & B3-4 & 12.5 & 38 & 50 & 0.02 & 10 & 2.7 & 0.21 & 0.55 \\
$\zeta^{1}$ Sco & B1.5 & 12.5 & 45 & 50 & 0.5 & 5 & 5.5 & 0.33 & 0.32 \\
HD 190603 & B1.5 & 15 & 49 & 55 & 0.5 & 5 & 5.5 & 0.33 & 0.40 \\
BP Cru & B1 & 10 & 55 & - & 0.5 & 3.5 & 4.8 & 2.0 & 0.40 \\
\hline
\end{tabular}

Notes. $R_{*}$ corresponds to $R\left(\tau_{\text {Ross }}=2 / 3\right)$, the $\mathrm{H} / \mathrm{He}$ ratio is given by number and $v_{\text {phot }}$ in terms of $v_{\text {sound }}$. Abundances are relative to solar values from Anders \& Grevesse (1989) and have uncertainties of, typically, $\sim 0.2$ dex; if we use the values from Asplund et al. (2006) as a reference the derived ratios need to be scaled by $1.49,1.86$ and 1.86 for $\mathrm{C}, \mathrm{N}$ and $\mathrm{O}$ respectively. The results presented here assume a distance of $1.75 \mathrm{kpc}$ for Cyg OB2 \#12 $(D M=11.21)$. Apart from the assumed distance of $1.64 \mathrm{kpc}$ for $\zeta^{1} \mathrm{Sco}(D M=11.07$, Sana et al 2006) we also provide corresponding stellar properties for the other distance estimate $(1.99 \mathrm{kpc}, D M=11.50)$ assumed in previous spectroscopic studies. For HD 190603 we display the stellar properties assuming a distance of $1.57 \mathrm{kpc}(D M=10.98)$ as well as those which would result if the object had the same $M_{V}$ as $\zeta^{1}$ Sco. Finally a distance of $3.04 \mathrm{kpc}$ (Kaper et al. 2006) is assumed for BP Cru.

Wind properties and clumping Unfortunately, the moderate reddening affecting Cyg OB2 \#12 prevents us from securing UV observations from which one might derive firm $v_{\infty}$ estimates. Furthermore, as with $\zeta^{1}$ Sco and HD 190603, Cyg OB2 \#12's stellar wind is not sufficiently dense that alternative $v_{\infty}$ diagnostic lines such as $\mathrm{H} \alpha$ or $\mathrm{He}_{\mathrm{I}} 10830 \AA$ can reach their full potentiallity and unambiguously yield the wind terminal velocity.

Highlighting this difficulty, Souza \& Lutz (1980) suggested a terminal velocity of $1400 \mathrm{~km} \mathrm{~s}^{-1}$, based on the presence of a blueshifted absorption feature in the $\mathrm{H} \alpha$ profile; a value which was subsequently used to obtain the mass-loss rate from radio (Abbott et al. 1981) and IR measurements (Leitherer et al. 1982). Subsequently, Klochkova \& Chentsov (2004) revised the estimate of the wind's terminal velocity significantly downwards. Based on higher resolution $\mathrm{H} \alpha$ observations they identified strong electron scattering wings extending up to $1000 \mathrm{~km} \mathrm{~s}^{-1}$, as well as blueshifted absorption up to $\sim 160 \mathrm{~km} \mathrm{~s}^{-1}$ which they attributed to the wind's true $v_{\infty}$. However our models indicate that this blueshifted feature does not directly reflect $v_{\infty}$ but rather results from the run of the density and ionization structures within the wind which shapes $\mathrm{H} \alpha$.

This is shown in Fig. 9, where models with $v_{\infty}$ ranging from 400 to $1000 \mathrm{~km} \mathrm{~s}^{-1}$ yield an absorption feature around $100-200 \mathrm{~km} \mathrm{~s}^{-1}$ to the blue of $\mathrm{H} \alpha$ in the resultant synthetic spectra. Moreover, values of $v_{\infty}$ significantly below $400 \mathrm{~km} \mathrm{~s}^{-1}$ appear too low to reproduce the high velocity line emission while, depending on the normalization errors of the $\mathrm{H} \alpha$ profile, values between 400 and $1000 \mathrm{~km} \mathrm{~s}^{-1}$ are potentially consistent. We also found that the blue absorption component of the He I $10830 \AA$ line is likewise not able to distinguish between $v_{\infty}=150 \mathrm{~km} \mathrm{~s}^{-1}$ and $v_{\infty}=1000 \mathrm{~km} \mathrm{~s}^{-1}$. We note that if the wind density of Cyg OB2 \#12 was a factor of two higher, the blue absorption component would develop up to the corresponding value of $v_{\infty}$.
Nevertheless, an upper limit on $v_{\infty}$ may be obtained by means of the $\mathrm{Br} \alpha / \mathrm{He}_{\mathrm{I}} 4.048 \mu \mathrm{m}$ complex. This is apparent in Fig. 9, where we see that ISO observations of this feature clearly resolve both components. Our models indicate that if $v_{\infty}$ is above $\sim 500 \mathrm{~km} \mathrm{~s}^{-1}$, both components are blended, while strong emission bluewards of $400 \mathrm{~km} \mathrm{~s}^{-1}$ is present, which is not seen in the data. On their own, these observations lack sufficient $\mathrm{S} / \mathrm{N}$ and spectral resolution to accurately constrain $v_{\infty}$, but in conjunction with $\mathrm{H} \alpha$ they jointly provide stronger constraints. Following this approach, we adopt $v_{\infty}=400 \mathrm{~km} \mathrm{~s}^{-1}$ for the remainder of the paper, while recognising that values between 300 and $1000 \mathrm{~km} \mathrm{~s}^{-1}$ cannot formally be discarded at present. Regarding this, we suggest that ground based high resolution spectra of $\operatorname{Br} \alpha$ and with sufficient $\mathrm{S} / \mathrm{N}$ to trace the line wings may constitute the best observational constraint available to estimate $v_{\infty}$.

Likewise, we make use of the shape of $\mathrm{H} \alpha$ and the $\operatorname{Br} \alpha / \mathrm{He}$ I $4.048 \mu \mathrm{m}$ complex to estimate $\beta$, the parameter characterizing the velocity law. We obtain $\beta=3$ indicating a relatively flat velocity field. Values of $\beta$ below 2 or above 4.5 can be ruled out from the line fits.

We are, however, unable to fully reproduce the blue shoulder of the $\mathrm{H} \alpha$ line, noting that this is the case irrespective of the terminal wind velocity adopted in the modeling. In this regard we highlight comparable discrepancies between the synthetic and observed $\mathrm{H} \alpha$ profiles for $\zeta^{1}$ Sco and HD 190603 (cf. an inability to reproduce the P Cygni absorption features in these stars; Figs. 6 and 7) while in many cases the model fits to BSGs presented by both Crowther et al. (2006) and Searle et al. (2008) also suffer similarly.

Regarding Cyg OB2 \#12, the lack of changes in polarisation through the line (Appendix B) strongly argues against any large scale wind asymmetries that might have been supposed to explain this feature. Hence we feel confident in the application of 

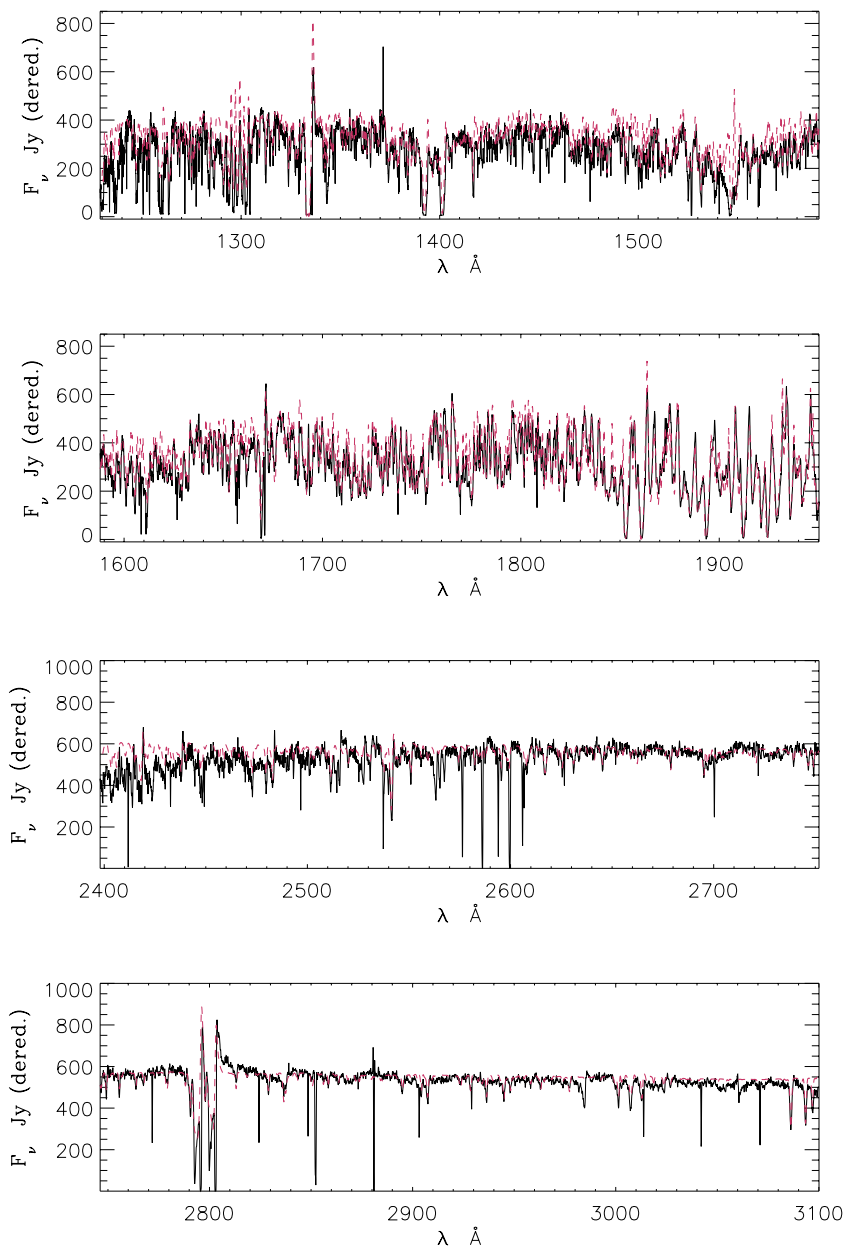

Fig. 5. Comparison of the synthetic (red dashed line) with the observational (black line) UV spectrum of $\zeta^{1}$ Sco. Note that the narrow lines that are not replicated in the model are interstellar in origin.

CMFGEN - which adopts spherical symmetry - to this and other stars in this study, while noting that this discrepancy might indicate a shortfall in the physics employed. Subject to this - and the preceding - caveats, we emphasise that the presence of this disagreement between model and observations does not affect our ability to determine the terminal velocity of the wind following the methodology described above ${ }^{5}$.

Finally, we were able to derive a mass-loss rate of $\dot{M}=$ $3.0 \times 10^{-6} M_{\odot} \mathrm{yr}^{-1}$ (Table 4). Of course this value is bound to the adopted $v_{\infty}$ and clumping law. Thus, if $v_{\infty}$ values of 300, 700 and $1000 \mathrm{~km} \mathrm{~s}^{-1}$ were adopted, fits of similar quality would be obtained for $\dot{M} \sim 2.5,4.0$ and $6.5 \times 10^{-6} M_{\odot} \mathrm{yr}^{-1}$ respectively.

The main observational constraints which set the run of the clumping law are the $\mathrm{H} \alpha-\beta$ and $\mathrm{Br} \alpha$ emission components and

\footnotetext{
5 For completeness we also explored the possibility that the discrepancy between observations and model could be due to the presence of (spatially unresolved) blueshifted nebular emission. Fitting an additional simple Gaussian profile to the blue emission "shoulder" in the $\mathrm{H} \alpha$ and $\beta$ lines, scaled assuming typical nebular line intensity ratios $(\mathrm{H} \alpha / \beta \sim 2.9$ and $\mathrm{H} \beta / \gamma \sim 2.2)$, resulted in significantly improved line fits. However, we caution that the adoption of such a solution appears premature at present as no other expected nebular emission lines such as $\left[\mathrm{N}_{\mathrm{II}}\right],\left[\mathrm{S}_{\mathrm{II}}\right]$ or $\left[\mathrm{O}_{\mathrm{I}}\right]$ are present in the spectrum of Cyg OB2 \#12; further adaptive optics or coronographic observations to search for compact nebular emission would be of value to determine if such an approach is physically well motivated.
}

the IR and submillimeter + radio continuum. An onset of the clumping at relatively high velocity $\left(\sim 200 \mathrm{~km} \mathrm{~s}^{-1}\right)$ is required to avoid strong emission in the above lines and too great an excess in the IR continuum. On the other hand, we find that a final clumping value of 0.04 is required to reproduce the submillimeter and radio continuum (see Fig. 2).

Our derived mass loss rate presents one of the major differences with respect to previous works. The significantly lower clumped mass loss rate of $\dot{M} \sim 3.0 \times 10^{-6} M_{\odot} \mathrm{yr}^{-1}$ corresponds to an unclumped $\left(\dot{M} / f^{0.5}\right)$ rate of $\sim 1.5 \times 10^{-5} M_{\odot} \mathrm{yr}^{-1}$; a factor of $\sim 3$ lower than that reported by Leitherer et al. (1982), due to the considerably smaller wind terminal velocity $\left(v_{\infty}\right)$ adopted (400 versus $1400 \mathrm{~km} \mathrm{~s}^{-1}$ ). The effects of the high degree of wind clumping found for Cyg OB2 \#12 mirror recent findings for other massive (evolved) stars (e.g. Najarro et al. 2009; Groh et al. 2009a,b).

Nevertheless, such a clumping corrected value is in excess of that found for the less luminous and massive B supergiants studied by Crowther et al. (2006) and Searle et al. (2008). For wind velocities $v_{\infty} \leq 400 \mathrm{~km} \mathrm{~s}^{-1}$ the clumping corrected mass loss rate approaches those of known LBVs (Fig. 11; Sect. 5), although the terminal velocity is comparable to BSGs of similar temperature. Adopting $v_{\infty} \sim 1000 \mathrm{~km} \mathrm{~s}^{-1}$ leads to a mass loss rate comparable to those of the $\mathrm{LBV}_{\mathrm{s}}$, but such a wind velocity is significantly greater than those determined for both LBVs and BSGs of equivalent spectral type. With respect to this, the lack of the low excitation metallic emission lines that characterise cool phase LBV spectra (Sect. 3) is a result of the extremely large radius found for Cyg OB2 \#12.

Abundances Fortunately, unlike stars such as HDE 316285 (Hillier et al. 1998), Cyg OB2 \#12 does not suffer from a $T_{\text {eff }} /$ He-abundance degeneracy. Since, as previously described, $T_{\text {eff }}$ can be accurately determined from e.g. the $\mathrm{Si}$ III/Si II or $\mathrm{O}_{\text {II/ }} / \mathrm{O}_{\text {I }}$ equilibria, we may therefore reasonably constrain the $\mathrm{He} / \mathrm{H}$ ratio. In Fig. 10 we overplot synthetic spectra constructed with a wide range of $\mathrm{He} / \mathrm{H}$ abundances on selected $\mathrm{He}_{\mathrm{I}}$ transitions; note that as expected the best constraints are provided by the weaker, non saturated, transtions. This reveals one of the fundamental results of our study - that Cyg OB2 \#12 demonstrates a solar $\mathrm{H} / \mathrm{He}=10$ ratio (by number). Unlike the rest of BHGs analysed in this paper (see Table 4), which clearly demonstrate surface helium enrichment, the He I lines of Cyg OB2 \#12 are best reproduced assuming no helium enrichment at all (noting that previous studies of BSGs adopted $\mathrm{H} / \mathrm{He}=5$ for all stars considered; Crowther et al. 2006; Searle et al. 2008). Furthermore, even better fits to some of the strong He I lines in the $R$ - (He I $6678 \AA$ ), I- (He I 8581 and $8845 \AA$ ) and Z-bands (He I $10830 \AA$ ) are obtained if we assume He to be underabundant. Moreover, we find that $\mathrm{H} / \mathrm{He} \sim 8.0$ appears to provide a robust lower limit to the abundance ratio, with lower values resulting in unacceptably poor fits to the data (Fig. 10).

We note that given the current stellar temperature and luminosity, the lack of He enrichment at the stellar surfaces is at odds with the predictions from evolutionary models and challenges present theory of stellar evolution of very massive stars. We discuss this somewhat unexpected finding, and the implications for the evolutionary state of Cyg OB2 \#12 in Sect. 5. From Table 4 we see indications of $\mathrm{CNO}$ processing ( $\mathrm{N}$ enhancement and $\mathrm{CO}$ depletion) in Cyg OB2 \#12, though to a lesser extent than for $\zeta^{1}$ Sco and HD 190603. This result is consistent with the derived $\mathrm{H} / \mathrm{He}$ ratios. 

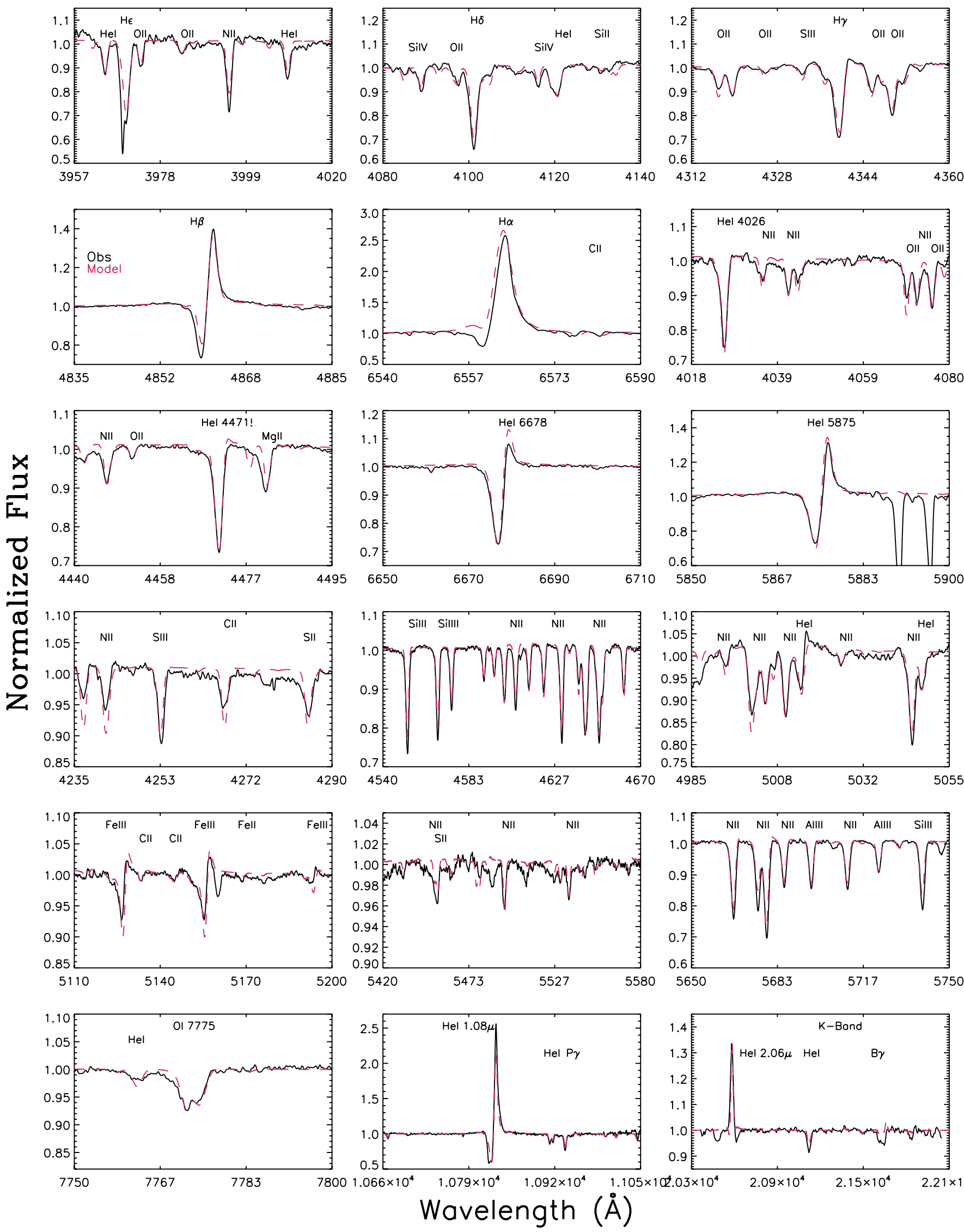

Fig. 6. Comparison of the synthetic spectrum of $\zeta^{1}$ Sco (red dashed line) with observational data for various transitions between $4090-22100 \AA$. Optical data correspond to the 2006 ESO NTT/EMMI run. Note that narrow unlabeled absoption lines are telluric in origin. 
J. S. Clark et al.: On the nature of the galactic early-B hypergiants
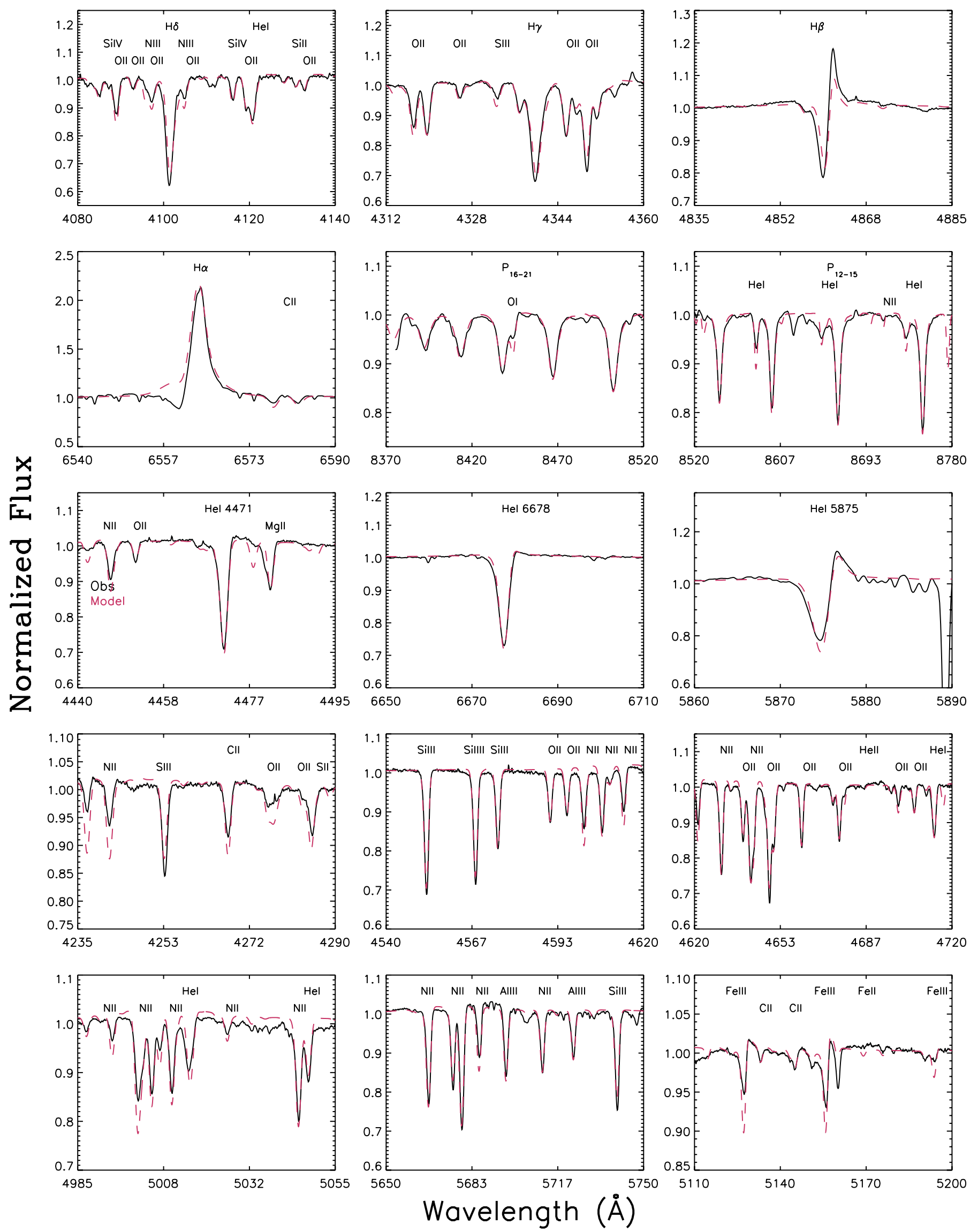

Fig. 7. Comparison of the synthetic spectrum of HD 190603 (red dashed line) to observational data. Optical data correspond to the FEROS data kindly provided by Otmar Stahl. As with $\zeta^{1}$ Sco (Figs. 7 and C.3), no long-term variability is present between different epochs (not shown). Note that narrow unlabeled absorption lines are telluric in origin. 

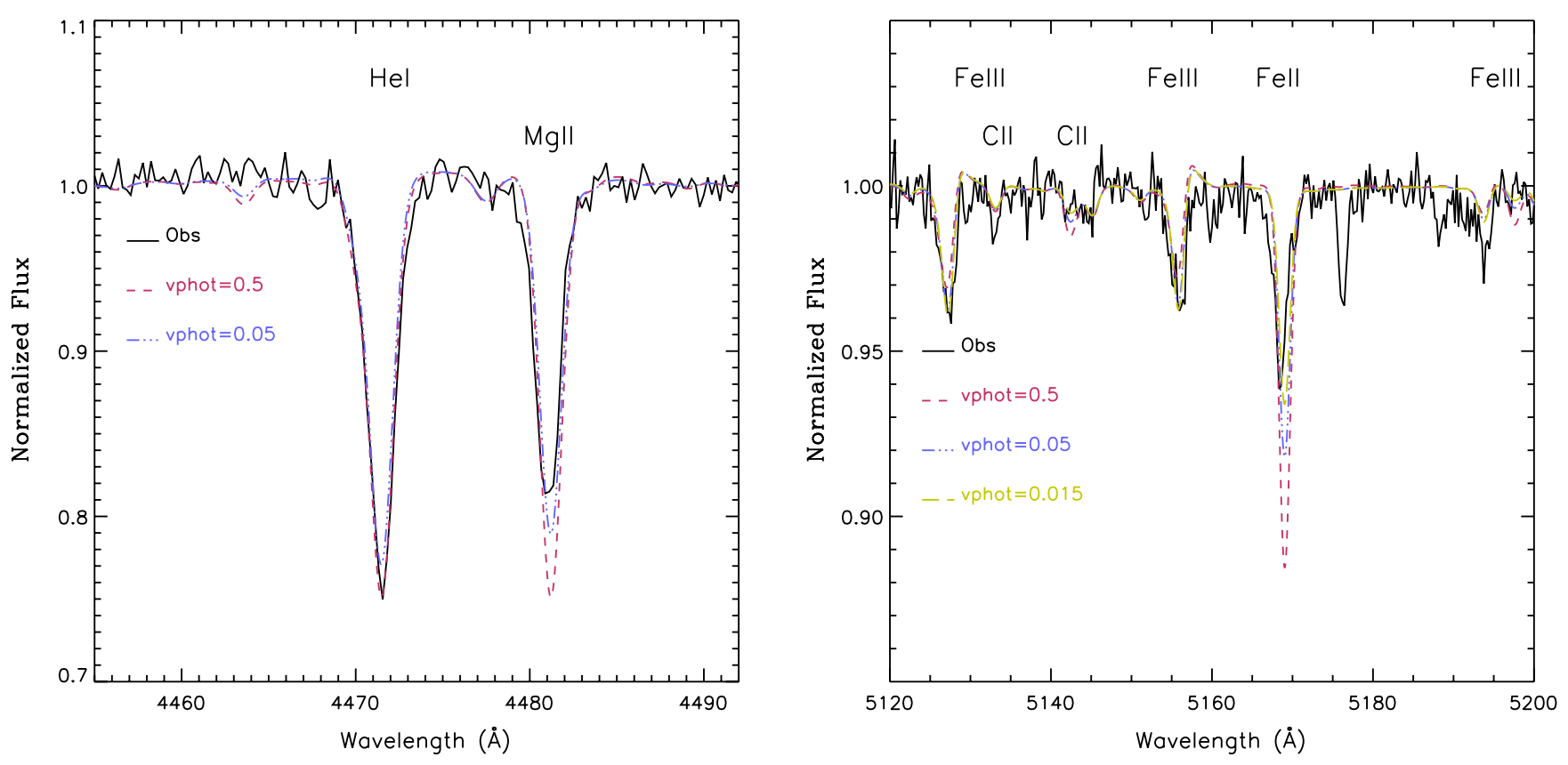

Fig. 8. Importance of the wind/photosphere transition region on the emergent spectrum of Cyg OB2 \#12. Left: influence of the transition velocity (in units of the sound speed, $v_{\text {sound }}$ ) on the spectral typing diagnostic lines $\mathrm{He}_{\mathrm{I}} 4471$ and $\mathrm{Mg}_{\text {II }} 4481 \AA$ A. Right: influence on the Fe III/Fe II ionization equilibrium diagnostic lines. The Fe II $5170 \AA$ A line is severely affected by the transition velocity while the Fe III lines remain roughly unaltered.
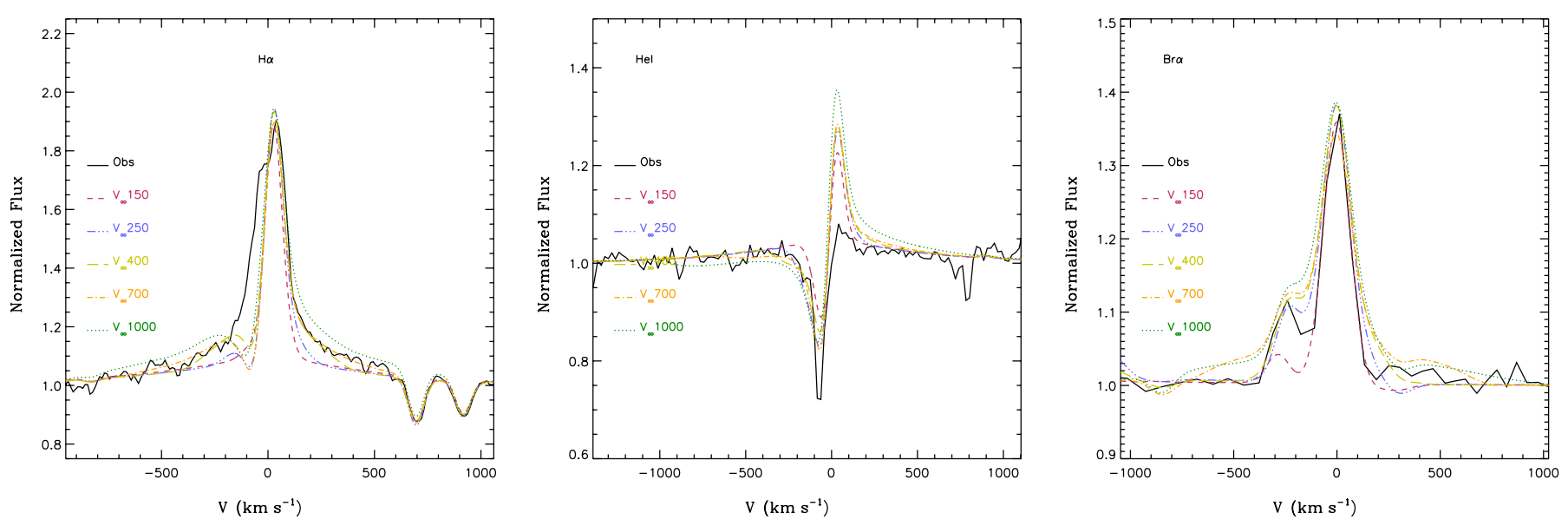

Fig. 9. Estimate of $v_{\infty}$ for Cyg OB2 \#12 from the $\mathrm{H} \alpha$ (left panel) and $\mathrm{He}$ I $10830 \AA$ lines (middle panel) and $\mathrm{B} \alpha / \mathrm{He}$ I $4.048 \mu \mathrm{m}$ complex (right panel).

\subsubsection{The unexpected luminosity of Cyg OB2 \#12}

A critical finding of this analysis is the rather extreme luminosity of Cyg OB2 \#12 (e.g. Fig. 12 and Sect. 5); are we overestimating this? An obvious explanation is that a chance alignment with its "host" association leads us to overestimate its distance. If this were the case, the large degree of reddening demonstrated by Cyg OB2 \#12 would either be due to a (narrow) sightline of anomalously high interstellar extinction towards it and only it but which nonetheless yielded Diffuse Interstellar Bands (DIBs) comparable to those found for other bona fide association members (e.g. Hanson 2003) - or would be circumstellar in origin (e.g. Massey \& Thompson 1991), thus permitting a more normal run of interstellar reddening for a lower distance. However, our model fits to the expanded SED show no evidence for an
IR excess at wavelengths shorter than $30 \mu \mathrm{m}$; suggesting that any circumstellar component would have to be significantly cooler than observed around known LBVs, where pronounced emission from warm dust is detected at $\leq 25 \mu \mathrm{m}$ (e.g. Egan et al. 2002; Clark et al. 2003a).

A second alternative is that it is a multiple system. Given the recent suggestion of a high binary fraction within Cyg OB2 (Kiminki et al. 2009) and the hard, luminous X-ray emission from Cyg OB2 \#12 (Albecete Columbo et al. 2007; Rauw et al. 2005 ) this would appear to be physically well motivated. To reduce the luminosity of Cyg OB2 \#12 such that it was marginally consistent with current theoretical predictions (Sect. 5) would require a companion(s) of equal luminosity. However clear spectroscopic signatures of binarity in terms of (i) radial velocity shifts; (ii) the presence of double lines; and/or (iii) the dilution 

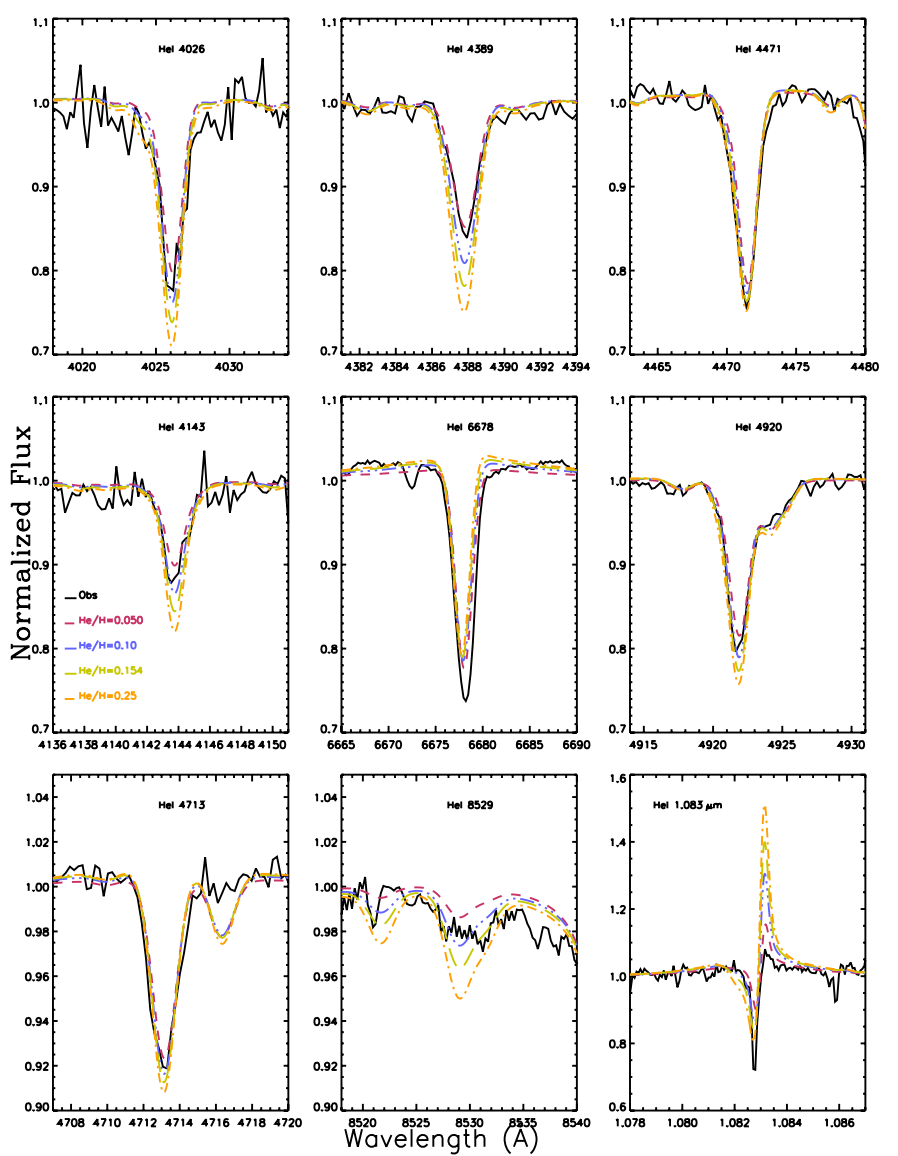

Fig. 10. Montage of selected He I lines in the spectrum of Cyg OB2 \#12 with synthetic spectra computed utilising different $\mathrm{He} / \mathrm{H}$ ratios overplotted.

of spectral features appear absent ${ }^{6}$. Moreover, the excellent model fit to the observed spectral energy distribution also excludes a companion of comparable luminosity but very different temperature.

While not fatal to a binary scenario, these observational constraints would require any putative binary companion of comparable luminosity to have an identical spectral type - to avoid its spectral signature being visible - and for the binary to either be very wide or seen face on - to avoid RV shifts in the spectrum. However, the temperature of the hard X-ray component $(k T=1.8 \mathrm{keV})$ implies, via the strong shock condition, that the companion would have to have a wind velocity of $\sim 1300 \mathrm{~km} \mathrm{~s}^{-1}$ - inconsistent with the emission arising in the wind collision zone produced by such a "twin" and necessitating the presence of a third, unseen star to yield the high energy emission. While we cannot exclude such a "finely tuned" hierarchical system, we find no compelling evidence for one either.

Lastly, one might suppose that Cyg OB2 \#12 were currently undergoing a long-term "LBV eruption" resulting in an increased luminosity. However, while the physical properties of such events are ill constrained we find the current physical properties to be distinct from those of quiescent LBVs (e.g. Sect. 5), while mass loss rates of $\gg 10^{-5} M_{\odot} \mathrm{yr}^{-1}$ are typically inferred for such stars in outburst (Clark et al. 2009). Therefore, while we may not formally exclude any of these possibilities,

\footnotetext{
${ }^{6}$ In particular we note that no (anti-phased) radial velocity shifts indicative of binarity are present in the additional emission components of the $\mathrm{H} \alpha, \beta$ and $\gamma$ lines.
}

we find no compelling observational evidence to support them either and hence consider it likely that the primary BHG within Cyg OB2 \#12 is indeed unexpectedly luminous in comparison to theoretical predictions. Moreover, even if its luminosity were reduced via one of these scenarios such that it was consistent with current evolutionary tracks, we note that the revised mass loss rate $\left(\dot{M} \propto d^{1.5}\right)$ and (unchanged) chemistry would still remain discrepant with respect to field BSGs and BHGs.

\section{2. $\zeta^{1}$ Sco and HD 190603}

For the analysis of $\zeta^{1}$ Sco we adopted a distance $d \sim 1.64 \mathrm{kpc}$, based upon the revised estimate for NGC 6231/Sco OB1 (Appendix C); for convenience we also show results for the slightly larger distance of $d \sim 1.9 \mathrm{kpc}$ adopted in previous works to enable a direct comparison(Crowther et al. 2006). Unfortunately, HD 190603 is not associated with a host cluster making the distance significantly more uncertain. For consistency to prior studies we adopt an identical distance $(d \sim$ $1.57 \mathrm{kpc}$; Crowther et al. 2006), while also presenting results under the assumption that it has an identical intrinsic visual magnitude to $\zeta^{1}$ Sco.

As in the case of Cyg OB2 \#12, several ionization equilibria could be used to determine the stellar temperature. Interestingly we could use three ionization stages of silicon ( $\mathrm{Si} \mathrm{IV} / \mathrm{Si} \mathrm{III/Si} \mathrm{II)}$ for both objects. In the case of HD 190603, our hottest object, the Fe II and $\mathrm{N}_{\mathrm{I}}$ lines are not detected so we used them in our models as indicators of a lower limit for $T_{\text {eff }}$. On the other hand, He II $4686 \AA$ A was weakly detected in HD 190603 allowing us to use as well the $\mathrm{He}$ II/He I ionization criterium.

Since UV spectra are available for both objects, the terminal velocity, $v_{\infty}$, could be accurately determined for both objects (e.g. Fig. 5). Given that we are fitting non-simultaneous observations from the UV to the IR we might expect to find some transitions in this wavelength range which are not well fitted by our synthetic spectra. This appears to be the case for C IV 1548-1551 $\AA$, which is significantly underestimated by our models. Moreover these, as well as the Si IV 1394-1403 UV lines, are very sensitive to X-rays in this parameter domain; thus they should be regarded with caution.

The main stellar properties of both objects are presented in Table 4. Uncertainties in stellar parameters are similar to those derived for Cyg OB2 \#12, except for the effective temperature, which is slightly better constrained $\Delta T_{\mathrm{eff}}= \pm 500 \mathrm{~K}$ and the terminal velocity with $\Delta v_{\infty}= \pm 50 \mathrm{~km} \mathrm{~s}^{-1}$. Errors on the $\mathrm{H} / \mathrm{He}$ ratio are of the order of $\sim 20 \%$. Interstellar reddening was also determined; for $\zeta^{1}$ Sco we found $E(B-V)=0.66$ and $R_{V}=3.3$ and for HD $190603 E(B-V)=0.70$ and $R_{V}=3.1$.

We found both stars to be less extreme than Cyg OB2 \#12 in terms of luminosity, although $\zeta^{1}$ Sco is still significantly in excess of the range of luminosities spanned by Galactic O9-B5 Ia/b supergiants (Searle et al. 2008; Crowther et al. 2006), even with the downwards revision of the distance. HD 190603 lies in the upper reaches of this distribution, but we caution that this could be subject to revision given the uncertainties in its distance estimate. As expected from their earlier spectral types, they are hotter than Cyg OB2 \#12, although the temperatures we find are slightly lower $(<1 \mathrm{kK})$ than previous estimates for these objects. These lower temperature estimates are due in part to the effect of the wind transition region (see Sect. 4.1) and to the use of all available ionization equilibria criteria. Following from these results, spectroscopic mass estimates are significantly lower than that of Cyg OB2 \#12 for both stars. 


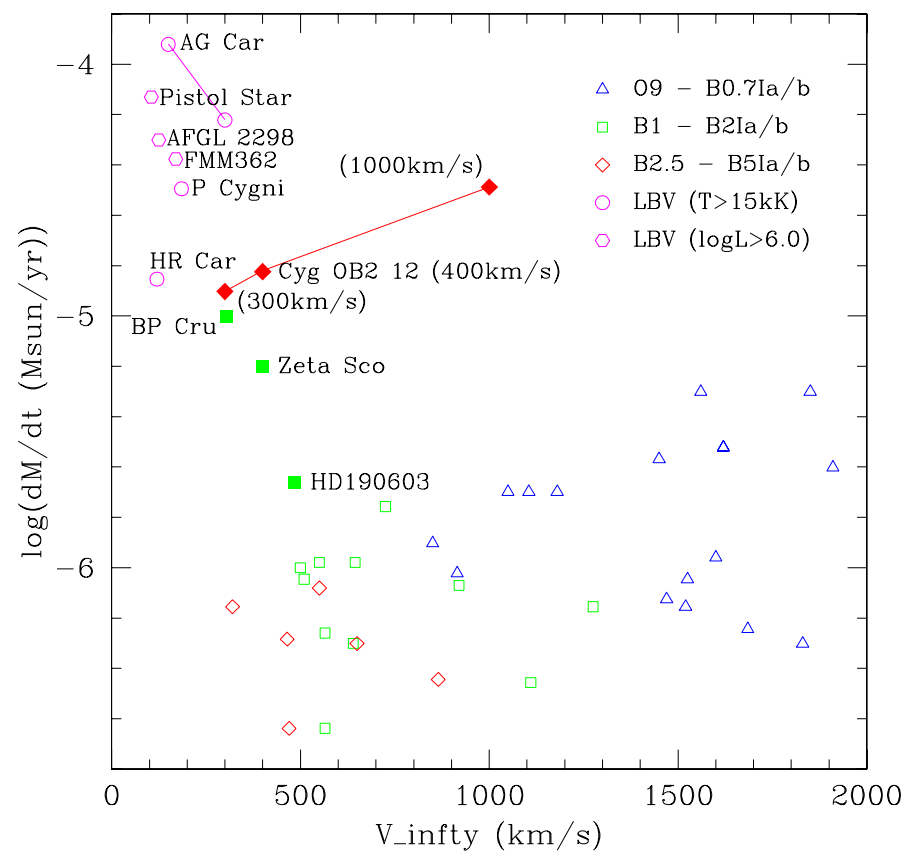

Fig. 11. Comparison of the wind properties of the BHGs to field BSGs (plotted according to spectral type), LBVs of temperature selected to match the BSGs $(\geq 15 \mathrm{kK})$ and those of comparable luminosity to Cyg OB2 \#12 $\left(\log \left(L / L_{\odot}\right)>6.0\right)$. Data from Crowther et al. (2006), Searle et al. (2008), Najarro et al. (1999, 2009), Clark et al. (2009) and Groh et al. (2009a, 2009b). Inclusion of later BSGs continues the trend to lower mass loss rates and wind velocities (e.g. Markova et al. 2008). Due to the uncertainty in the wind velocity for Cyg OB2 \#12, we present 3 pairs of possible values for $v_{\infty}=300,400$ and $1000 \mathrm{~km} \mathrm{~s}^{-1}$ (Sect. 4.1).

Terminal wind velocities are similar to Cyg OB2 \#12, but lie at the lower range of values found for BSGs of comparable spectral type; in contrast the clumping corrected mass loss rate for HD 190603 lies at the upper range of those found for B1-2 Ia/b supergiants while that of $\zeta^{1}$ Sco is considerably in excess of this range (Fig. 11). These compare favourably with previous estimates for these stars (Searle et al. 2008; Crowther et al. 2006; Markova \& Puls 2008); although as with Cyg OB2 \#12, the high degree of clumping required to fit the spectra provide a significant downwards revision of absolute mass loss rates between this and prior studies. Values of $\log g$ are also lower than previous estimates of both stars, due to the greater accuracy afforded us by the inclusion of the higher Balmer and Paschen lines in the spectroscopic datasets.

Finally, in contrast to Cyg OB2 \#12, but in line with estimates for field Galactic BSGs, we find a moderate $\mathrm{H}$-depletion (or He-enrichment; $\mathrm{H} / \mathrm{He} \sim 5$ by number) for both stars as well as evidence for stronger $\mathrm{CNO}$ processing (higher $\mathrm{N}$ ).

\section{Discussion}

While the galactic BHG population spans over an order of magnitude in luminosity (Fig. 12), when combined with previous modeling results for BP Cru (Table 4; Kaper et al. 2006), our analyses show the early $\mathrm{B} 1-4 \mathrm{Ia}^{+}$hypergiants to be, as expected, rather luminous evolved stars, with HD 80077 and Cyg OB2 \#12 appearing to lie above the empirical HD limit (Fig. 12; Marco \& Negueruela 2009). In comparison to Galactic BSGs of the same spectral subtypes they are overluminous and

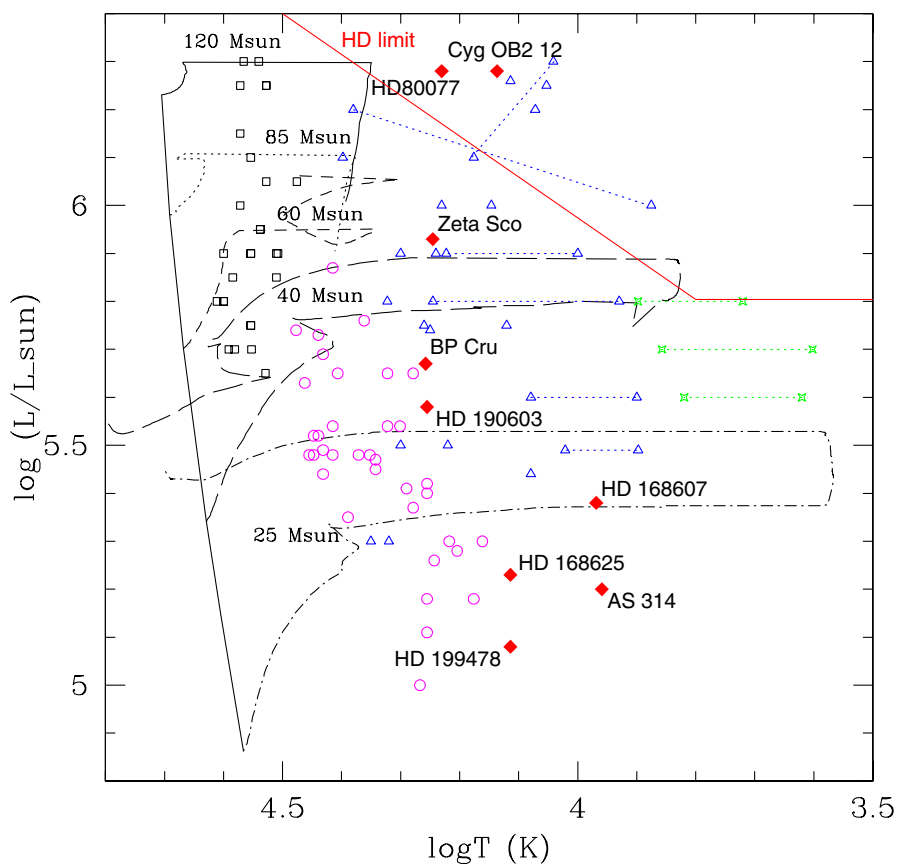

Fig. 12. HR diagram with the positions of the BHGs (red diamonds) plotted with respect to the evolutionary tracks of Meynet \& Maeder (2005) and the empirical HD limit. The position of HD 80077 assumes membership of Pismis 11 and hence a distance of $3.5 \mathrm{kpc}$ (Marco \& Negueruela 2009). The positions of other evolved stellar populations are also indicated: black squares - WN7-9h, O4-6 Ia and O4-6 If $^{+}$stars within the Arches; purple circles - galactic field BSGs; blue triangles - (candidate) LBVs and WNL stars; green stars - YHGs. Data from Martins et al. (2007, 2008), Crowther et al. (2006), Searle et al. (2008) and Clark et al. (in prep.).

support significantly higher mass loss rates, with wind terminal velocities at the lower end of the range populated by the BSGs.

\subsection{The evolutionary status of BHGs}

Subject to these observational constraints how may single (early) BHGs be understood in evolutionary terms? Several authors have presented evolutionary sequences for massive (>30 $\left.M_{\odot}\right)$ stars, including Langer et al. (1994), Crowther et al. (1995), Meynet \& Maeder (2005) and Martins et al. (2007, 2008). After the initial O-type main-sequence phase, the recent empirical evolutionary scheme of the latter authors may be summarized as:

- $\sim 60-120 M_{\odot}:$ O Ia $\rightarrow \mathrm{OIaf}^{+} \rightarrow \mathrm{WNL}+$ abs $\rightarrow \mathrm{WN7}$

- $\sim 30-60 M_{\odot}: \mathrm{O} / \mathrm{B}$ Ia $\rightarrow \mathrm{WN} 9-11 \mathrm{~h} \rightleftharpoons \mathrm{LBV} \rightarrow \mathrm{WN} 8 \rightarrow$ $\mathrm{WN} / \mathrm{C}$.

While not explicit in the above scheme, the properties of LBVs such as AG Car (Groh et al. 2011) and the Pistol star (Najarro et al. 2009) strongly imply that even very massive $M_{\text {ini }}>60 M_{\odot}$ stars may experience an LBV phase. Moreover, it is likely that a further subdivision will occur for $M_{\text {ini }}<40 M_{\odot}$ to accommodate the high luminosity $\left(\log \left(L / L_{\odot}\right) \sim 5.8\right)$ cool hypergiants present in clusters such as Westerlund 1 (Wd 1; Clark et al. 2005b):

- $\sim 30-40 M_{\odot}: \mathrm{O} \rightarrow \mathrm{OB} \mathrm{Ia} \rightarrow \operatorname{cool~Ia}{ }^{+} \rightarrow \mathrm{WN} \rightarrow \mathrm{WC}$.

Noting that the evolution of spectral classification through the red loop and the final state reached prior to $\mathrm{SN}$ is particularly uncertain. 
Given that the physical properties of the BHGs appear more extreme than the field BSGs and that they appear co-located with the LBVs on the HR diagram (Fig. 12) a close physical association is suggested and we propose that the early BHGs - with the notable exception of Cyg OB2 \#12 - are the immediate evolutionary progenitors of LBVs for stars of $\sim 40-60 M_{\odot}$. Thus, they would form lower mass analogues of the 04-6 $\mathrm{Iaf}^{+}$stars in the Arches, which Najarro et al. (2004) and Martins et al. (2008) demonstrate are intermediate between the O4-6 Ia and WN7-9ha stars both in terms of the degree of (increasing) chemical evolution and also wind properties, with advancing evolutionary state leading to a reduction in terminal wind velocities coupled with an increase in mass loss rate.

The BHGs appear to follow an analogous evolutionary template, with wind properties approaching those of the LBVs (Fig. 11). Moreover, the BHGs also appear less chemically evolved than (candidate) $\mathrm{LBVs}^{7}$, again suggesting that they occupy an earlier evolutionary phase. Such an hypothesis appears bolstered by the apparent lack of long-term(secular) variability demonstrated by the early-B HGs (Sect. 3), although we are aware that bona fide LBVs such as P Cygni may also encounter long periods of quiescence.

We emphasise that this evolutionary scheme is appropriate for single stars, but it also appears possible that early-B HGs may form via binary interaction and, through such a channel, potentially from lower mass stars. Wellstein \& Langer (1999) present the results of simulations that show the properties of the known binary BP Cru are consistent with quasi-conservative mass transfer in a $26 M_{\odot}+25 M_{\odot}$ progenitor system. Likewise, the BHG/WNVL transitional object Wd1-13 is the primary of a $23.2 M_{\odot}+35.4 M_{\odot} 9.27$ day period binary (Ritchie et al. 2010). This configuration is too compact for the primary to have passed through a BSG phase, and hence presumably formed via an episode of binary mediated mass loss (Sect. A.5), with a likely progenitor mass of $\sim 35 M_{\odot}$. In this respect we highlight that Wd1-13 appears spectroscopically distinct from the BHGs considered here, showing pronounced (variable) emission lines in the Paschen series (Fig. A.2 and Ritchie et al. 2010).

Finally, is it possible to place the later (lower luminosity) BHGs into comparable sequences? The consistency of the population of B1-3 Ia and cooler B5-9 $\mathrm{Ia}^{+}$stars within Wd 1 with theoretical evolutionary isochrones (Negueruela et al. 2010) suggests an extension of the paradigm that BHGs are the more physically extreme direct descendants of hotter O/BSGs to lower progenitor masses $\left(\sim 35 M_{\odot}\right)$. However, the placement of these BHGs in a pre- or post-RSG/LBV phase is currently uncertain in the absence of tailored abundance analyses - ESO/VLT observations currently underway will allow this to be directly addressed. Finally, following previous suggestions in the literature (Sect. A.6) - and again in the absence of quantitative analyses it appears likely that lower luminosity $\left(\log \left(L / L_{\odot}\right)<5.5\right)$ BHGs are post-RSG objects possibly encountering an LBV phase.

\subsection{Constraints from the host stellar aggregate}

Do other observations support such an evolutionary scheme? Following the above discussion, the stellar populations of both the Arches and Wd 1 are clearly consistent. However we would also predict the presence of early BHGs within the Quintuplet

\footnotetext{
${ }^{7} \mathrm{HD} 316285(\mathrm{H} / \mathrm{He} \sim 1.5)$, AG $\mathrm{Car}(\mathrm{H} / \mathrm{He} \sim 2.3)$, W243 (H/He 5), P Cygni $(\mathrm{H} / \mathrm{He} \sim 3.3)$, FMM362 $(\mathrm{H} / \mathrm{He} \sim 2.8)$ and the Pistol Star (H/He 1.5; Hillier et al. 1998; Groh et al. 2009a; Ritchie et al. 2009; Najarro et al. 2001, 2009, respectively).
}

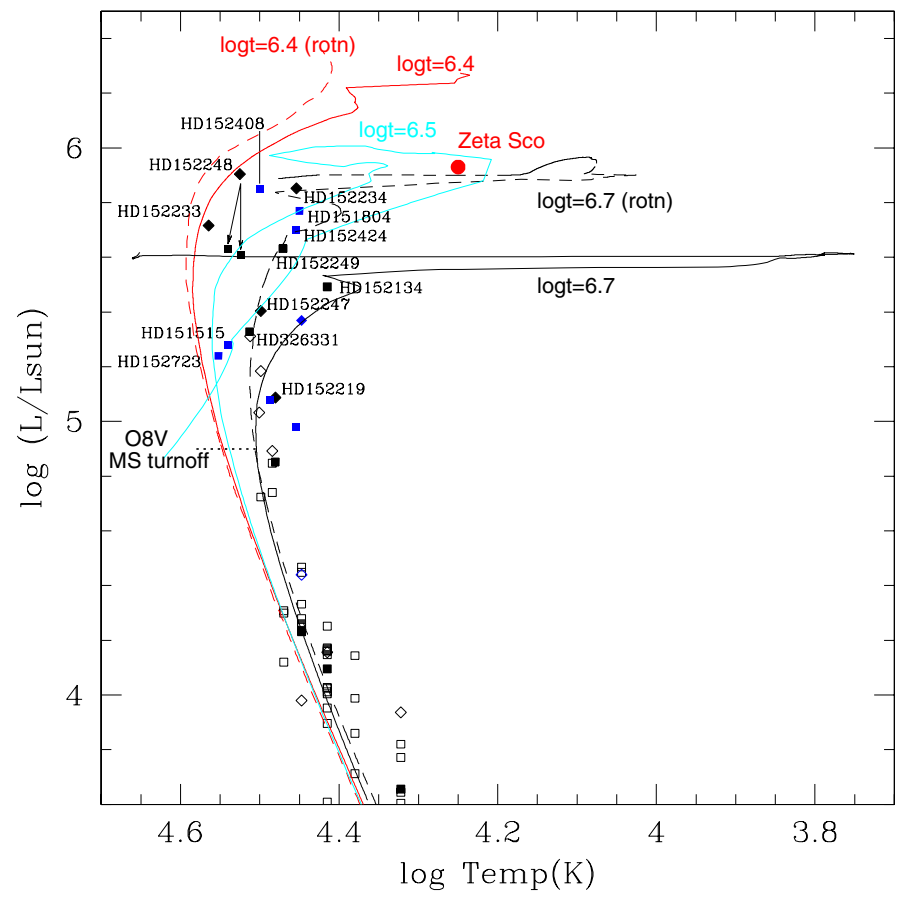

Fig. 13. HR diagram for NGC 6231 (black symbols) and the wider Sco OB1 association (blue symbols) with the non rotating isochrones of Schaller et al. (1992; solid lines) and the rotating isochrones of Meynet \& Maeder (2005; dashed line) overplotted. Open and filled symbols represent main sequence and evolved stars, while diamonds indicate binarity. For HD 152248 we plot the position of both the individual components and also that of the integrated system. See Appendix B for details of contruction.

cluster, which is likely to be intermediate in age between these clusters (Figer et al. 1999). While neither these authors nor Liermann et al. (2009) report the presence of BHGs, we note that e.g. LHO96, 100 and 146 - which are classified as O6-8. If by Liermann et al. - have $K$ band spectra identical to that of $\zeta^{1}$ Sco (Fig. 6). Simply applying the bolometric correction derived from our analysis of $\zeta^{1}$ Sco yields an estimated $\log \left(L / L_{\odot}\right) \sim 6.0-6.3$ for these stars; directly comparable to the Pistol Star and FMM362 (Najarro et al. 2009) and hence consistent with a pre-LBV BHG identification. Further high $\mathrm{S} / \mathrm{N}$ and resolution observations of these stars would permit quantitative testing of this assertion by virtue of an accurate determination of temperature, luminosity and wind properties.

Encouragingly, stars with a similar $K$ band spectral morphology are also present in the Galactic Centre cluster, where they too are found to be of higher temperature and possess faster, lower mass loss rate winds than the more evolved WNL - and candidate LBV - stars present (Najarro et al. 1997; Martins et al. 2007).

Importantly, are the properties of $\zeta^{1}$ Sco and Cyg OB2 \#12 and their host stellar aggregates consistent with this evolutionary hypothesis? Unfortunately, observations of Cyg OB2 and NGC 6231/Sco OB1 raise the possibility that the stellar populations in both associations are non-coeval (Negueruela et al. 2008; Appendix C). Nevertheless, the physical properties of $\zeta^{1}$ Sco appear consistent with the population of (initially) rapidly rotating, 5 Myr-old stars present within NGC 6231 (Fig. 13 and Appendix B; noting that the low $v_{\mathrm{e}} \sin i$ of $\zeta^{1}$ Sco may be understood as a combination of spindown and/or an unfavourable inclination) or a somewhat younger ( 3.2-4 Myr) population of less rapidly rotating objects that may also be 


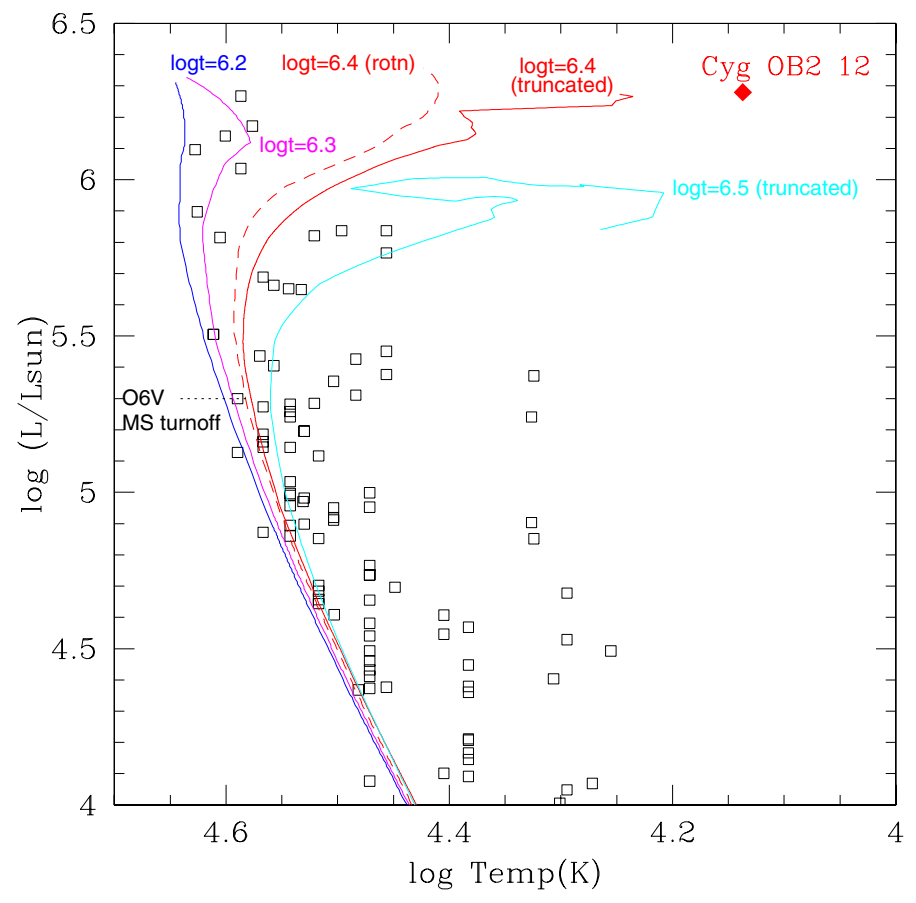

Fig. 14. HR diagram for Cyg OB2 with the non rotating isochrones of Schaller et al. (1992; solid lines) and the rotating isochrone of (Meynet $\&$ Maeder 2005; dashed line) overplotted. Data from Negueruela et al. (2008) and Hanson et al. (2003); the reader is refered to these studies for the positions of individual stars, which have been omitted here for reasons of clarity.

present (Appendix B). This would imply a progenitor mass of $\sim 40-60 M_{\odot}$ for $\zeta^{1}$ Sco, depending on age and initial rotation, broadly consistent with the current spectroscopic mass of $\sim 36 M_{\odot}$.

Finally, we address Cyg OB2 \#12. As highlighted previously, this star appears difficult to accomodate in the current evolutionary scheme due to the combination of high luminosity and low temperature displacing it from any theoretical isochrones applicable to Cyg OB2 (Fig. 14; Negueruela et al. 2008). While its co-location in the HR diagram with bona fide LBVs such as the Pistol Star, FMM 362 and AFGL 2298 (Fig. 12) suggests a similar nature, the long-term stability combined with its relatively unevolved chemsitry - and critically solar $\mathrm{H} / \mathrm{He}$ ratio - appear to exclude such an identification. We are thus currently unable to place Cyg OB2 \#12 into a coherent evolutionary scheme, noting that with a current spectroscopic mass of $\sim 110 M_{\odot}-$ significantly in excess of both the Pistol Star and FMM 362 (Najarro et al. 2009) - one might expect it to evolve through a much hotter O4-6I/ $\mathrm{Iaf}^{+} / \mathrm{WNLha}$ evolutionary sequence.

In this respect, the results of a quantative analysis of HD 80077 would be of particular interest, given its apparent similarly extreme luminosity (Marco \& Negueruela 2009). Such an analysis has been performed for the M $33 \mathrm{~B}^{-\mathrm{Ia}^{+}}$star [HS80] 110 A by Urbaneja et al. (2005) which yielded stellar parameters that, like Cyg OB2 \#12, both place it above the HD-limit and appear to distinguish it from known $\mathrm{LBVs}^{8}$. This finding appears to suggest that there might indeed be an hitherto unidentified (but rarely traversed?) evolutionary pathway that leads to the production of very luminous but comparately cool BHGs at very high stellar masses.

\footnotetext{
$\overline{{ }^{8} \log \left(L / L_{\odot}\right) \sim 6.5, T_{\text {eff }} \sim 2} 1 \mathrm{kK}, v_{\infty} \sim 750 \mathrm{~km} \mathrm{~s}^{-1}$ and $\dot{M}=1.06 \times$ $10^{-5} M_{\odot} \mathrm{yr}^{-1}$.
}

\section{Conclusions}

In order to determine the physical properties and hence evolutionary state of Galactic early-B hypergiants, we have undertaken new quantitative analyses of Cyg OB2 \#12, $\zeta^{1}$ Sco and HD 190603 and employ previous model results for BP Cru. Synthetic spectra and SEDs were calculated and compared to comprehensive UV/optical-radio spectroscopic and photometric datasets compiled from the literature and supplemented by new and previously unpublished observations. Building on this effort, we also constructed exhaustive spectroscopic and photometric histories for all Galactic BHGs in order to test the assertion that they are physical identifiable with LBVs.

Turning first to stellar variability and both the early- and lateB HGs demonstrated the rapid ( days) spectroscopic and photometric variability that is symptomatic of the aspherical wind substructure and photospheric pulsations that characterises luminous early stars. While low-luminosity, late-B HGs have long be known to undergo LBV excursions (e.g. HD 160529) we found no evidence for such secular variability amongst the early, high luminosity BHGs, with the possible exception of $\zeta^{1}$ Sco in the sparse photometric data obtained prior to the 20th Century. In this regard it is interesting that both Cyg OB2 \#12 and HD 80077 appear to lie above the empirical HD limit.

Model results reveal that the early-B HGs have physical properties - luminosity, wind terminal velocity, mass loss rate and chemical abundances - intermediate between Galactic BSGs and LBVs of comparable temperature, suggesting they provide a link between both evolutionary phases for stars of inital mass between $\sim 40-60 M_{\odot}$. In this respect, they would play a similar role to the more massive and luminous O4-6 $\mathrm{Iaf}^{+}$objects for stars in the $\sim 60-120 M_{\odot}$ range (Najarro et al. 2004; Martins et al. 2008). The simultaneous presence of populations of early B1-3 Ia supergiants and mid-late $\mathrm{B} 5-9 \mathrm{Ia}^{+}$BHGs within Wd 1 suggest that BHGs are potentially the immediate descendents of stars with initial masses as low as 30-35 $M_{\odot}$. However, the presence of early-B HGs within this cluster indicates that a parallel channel may also lead to this phase via close binary evolution.

Nevertheless, Wd 1 and the Quintuplet provide direct observational tests of the evolutionary sequence proposed; in the former cluster the BHGs should be more chemically evolved than the BSG population but less than the RSGs that are present, while in the latter cluster one would expect to find a population of early BHGs; observations aimed at verifying both hypotheses will be undertaken later this year.

The apparent physical association of $\zeta^{1}$ Sco with NGC 6231/Sco OB1 also allows us to test this scenario. Encouragingly, we find that the physical properties derived from our analysis are consistent with the observed stellar population of this region and in turn with theoretical isochrones for stars of between 3.2-5 Myr age - further discrimination is difficult in the absence of an absolute rather than projected rotational velocity.

Conversely, the combination of extreme luminosity and cool temperature of Cyg OB2 \#12 is inconsistent with theoretical isochrones, assuming membership of Cyg OB2. Given its colocation with a handful of bona fide LBVs above the HD limit it might be supposed to be a similar object, but a combination of (i) a lack of the characteristic LBV variability; (ii) extreme current stellar mass; and (iii) lack of chemical evolution differentiate it from these. We are therefore currently unable to place this star in a consistent evolutionary scheme, noting that Negueruela et al. (2008) also find that a number of the hottest and most luminous stars within Cyg OB2 are difficult to accommodate in 
a formation scenario for the association where the most recent epoch of star formation occurred $\sim 2.5$ Myr ago.

Nevertheless, we note that the stability of Cyg OB2 \#12 for the last century would appear to indicate that the HD limit does not appear to define a region of the HR diagram utterly inimical to the presence of massive stars, but a combination of (pulsational?) instabilities and extreme mass loss rates presumably prevent stars residing above it for large fractions of their lifetime. Indeed, from Fig. 12 we may see that in addition to highly luminous BHGs such as Cyg OB2 \#12 the empirical HD limit appears to be delineated by LBVs and other closely related (variable) stars such as the cool hypergiants and the WNVLs, further suggesting that it should not be regarded as a firm barrier to stellar stability, nor the location at which the onset of instabilities occurs. In this regard the $\mathrm{B} 1 \mathrm{Ia}^{+}$star [HS80] 110A and the cool F-hypergiant B324 (both located in M33) are of considerable interest; like Cyg OB2\#12, the combination of temperatures and luminosities they demonstrate are not replicated by current evolutionary tracks and place both stars well above the HD limit (Urbaneja et al. 2005; Clark et al. 2012). Quantitative modeling of the Galactic BHG HD 80077 would therefore be of considerable interest to see whether this too violated the HD limit.

Moreover, despite residing above the HD limit, we find an Eddington parameter, $\Gamma_{\text {Edd }}$, of only $\sim 0.41$ (and $\Gamma_{\text {Total }} \sim 0.56$ at $R\left(\tau_{\text {Ross }}=2 / 3\right)$ for Cyg OB2 \#12); below the Eddington limit. Unfortunately, in the absence of an inclination we may not determine how close Cyg OB2 \#12 is to the rotationally modified Eddington limit, although we note that the Eddington parameter for it is lower than Groh et al. (2011) found for AG Car at any epoch. Given that AG Car is also a known rapid rotator but has not been observed at, or to have exceeded, the rotationally modified Eddington limit we suspect the same to be true for Cyg OB2 \#12. From this we might therefore conclude that the HD-limit is not the direct result of a star (b)reaching the rotationally modified Eddington limit. Indeed, Groh et al. (2011) show that AG Car most closely approaches the rotationally modified Eddington limit during its hot, compact phases when, in contrast to its cool extended state, it lies beneath the HD limit (Fig. 12).

Acknowledgements. J.S.C. acknowledges support from an RCUK fellowship. This research is partially supportedby the Spanish Ministerio de Ciencia e Innovación (MICINN) under grants AYA2008-06166-C03-02/03, AYA201021697-C05-01/05 and CSD2006-70. M.A.U. acknowledges support by NSF under grant AST-10088798 We also thank John Hillier for providing the CMFGEN code and Dan Kiminki, Otmar Stahl, Philip Dufton and Sergio Simón-Díaz for providing electronic versions of published and unpublished stellar spectra.

\section{References}

Abbott, D. C., Bieging, J. H., \& Churchwell, E. 1981, ApJ, 250, 645

Abbott, D. C., Telesco, C. M., \& Wolff, S. C. 1984, ApJ, 279, 225

Adelman, S. J., \& Albayrak, B. 1997, IBVS, 4541, 1

Adelman, S. J., Yuce, K., \& Engin, S. 2000, IBVS, 4946, 1

Ahmad, I. I. 1952, ApJ, 115, 341

Albacete Columbo, J. F., Flaccomio, E., Micela, G., Sciortino, S., \& Damiani. F. 2007, A\&A, 464, 211

Allen, D. A. 1973, MNRAS, 161, 145

Anders, E., \& Grevesse, N. 1989, GeCoA, 53, 197

Andrews, P. H. 1968, MmRAS, 72, 35

Andrillat, Y., Jaschek, C., \& Jaschek, M. 1995, A\&AS, 112, 475

Ankay, A., Kaper, L., de Bruijne, J. H. J., et al. 2001, A\&A, 370, 170

Asplund, M., Grevesse, N., \& Jacques Sauval, A. 2006, NuPhA, 777, 1

Baume, G., Vázquez, R. A., \& Feinstein, A. 1999, A\&AS, 137, 233

Barsukova, E. A., Lebedeva, I. A., Chargejshvili, K. B., \& Chentsove, E. L. 1982, AISAO, 16, 34

Beals, C. S. 1946, JRASC, 40, 156

Becker, R. H., \& White, R. L. 1985, ASSL, 116, 139

Benaglia, P., Vink, J. S., Martí, J., et al. A\&A, 2007, 467, 1265

Bidelman, W. P. 1954, PASP, 66, 249
Bieging, J. H., Abbott, D. C., \& Churchwell, E. B. 1989, ApJ, 340, 518

Bisiacchi, G. F., Firmani, C., Ortega, R., \& Peniche, R. 1976, Rev. Mex. Astron. Astrofis., 2, 13

Blanco, V. M., Demers, S., Douglass, G. G., \& FitzGerald, M. P. 1968, Publ. US Naval Obs. 21

Blum, R. D., Ramond, T. M., Conti, P. S., Figer, D. F., \& Sellgren, K. 1997, AJ, 113,1855

Bohannan, B., \& Crowther, P. A. 1999, ApJ, 511, 374

Bord, D. J. 1979, A\&A, 77, 309

Botto, P., \& Hack, M. 1962, MmSAI, 33, 159

Bouigue, R., Boulon, J., \& Pedoussaut, A. 1961, Ann. Observ. Astr. Meteo. Toulouse, 28, 33

Bromage, G. E. 1971, Nature, 230, 172

Burnichon, M. L., \& Garnier, R. 1976, A\&AS, 24, 89

Burki, G. 1978, A\&A, 65, 357

Burki, G., Heck, A., Cassatella, A., \& Binchi, L. 1982, A\&A, 107, 205

Buscombe, W. 1962, MNRAS, 124, 189

Buscombe, W., \& Kennedy, P. M. 1968, MNRAS, 139, 341

Buscombe, W., \& Kennedy, P. M. 1969, MNRAS, 143, 1

Cannon, A. J. 1901, Harvard Obs. Ann., 28, part 2, 177, note 43

Chaldu, R., Honeycutt, R. K., \& Penston, M. V. 1973, PASP, 85, 87

Chentsov, E. L. 1955, Ap\&SS, 232, 217

Chentsov, E. L., Ermakov, S. V., Klochkova, V. G., et al. 2003, A\&A, 397, 1035

Clark, J. S., Egan, M. P., Crowther, P. A., et al. 2003, A\&A, 412, 185

Clark, J. S., Larionov, V. M., \& Arkharov, A. 2005a, 435, 239

Clark, J. S., Negueruela, I., Crowther, P. A., \& Goodwin, S. P. 2005b, A\&A, 434 949

Clark, J. S., Muno, M. P., Negueruela, I., et al. 2008, A\&A, 477, 147

Clark, J. S., Crowther, P. A., Larionov, V. M., et al. 2009, A\&A, 507, 1555

Clark, J. S., Ritchie, B. W., \& Negueruela, I. 2010, A\&A, 514, 87

Clark, J. S., Arkharov, A., Larionov, V., et al. 2011, BSRSL, 80, 361

Clark, J. S., Castro, N., Garcia, M., et al. 2012, A\&A, 541, A146

Clarke, A. J., Oudmaijer, R. D., \& Lumsden, S. L. 2005, MNRAS, 363, 1111

Code, A. D., \& Houck, T. E. 1958, PASP, 70, 261

Comerón, F., Pasquali, A., Rodighiero, G., et al. 2002, A\&A, 389, 874

Conti, P. S., \& Howarth, I. D. 1999, MNRAS, 302, 145

Contreras, M. E., Rodriguez, L. F., Gomez, Y., \& Velazquez, A. 1996, ApJ, 469, 329

Contreras, M. E., Montes, G., \& Wilkin, F. P. 2004, Rev. Mex. Astron. Astrofis., 40,53

Cousins, A. W. J. 1952, MNSSA, 11, 9

Crawford, D. L., Barnes, J. V., \& Golson, J. C. 1971, AJ, 76, 1058

Crowther, P. A., \& Evans, C. J. 2009, A\&A, 503, 985

Crowther, P. A., Smith, L. J., Hillier, D. J., \& Schmutz, W. 1995, A\&A, 293, 427

Crowther, P. A., Lennon, D. J., \& Walborn, N. R. 2006, A\&A, 446, 279

Dachs, J., Maitzen, H.-M., Moffat, A. F. J., Sherwood, W. A., \& Stift, M. 1977, A\&A, 56, 417

Davies, B., Oudmaijer, R. D., \& Vink, J. S. 2005, A\&A, 439, 1107

Dessart, L., \& Hillier, D. J. 2010, MNRAS, 405, 2141

Drout, M. R., Massey, P., Meynet, G., Tokarz, S., \& Caldwell, N. 2009, ApJ, 703, 441

Egan, M. P., Price, S. D., \& Gugliotti, G. M. 2001, BAAS, 34561

Egan, M. P., Clark, J. S., Mizuno, D. R., et al. 2002, ApJ, 572, 288

ESA, The Hipparcos and Tycho Catalogues, 1997, ESA SP, 1200

Evans, D. S., Menzies, A., \& Stoy, R. H. 1959, MNRAS, 119, 638

Fraser, M., Dufton, P. L., Hunter, I., \& Ryans, R. S. I. 2010, MNRAS, 404, 1306

Feinstein, A. 1968, ZA, 68, 29

Feinstein, A., \& Marraco, H. G. 1979, AJ, 84, 1713

Fernie, J. D. 1983, ApJS, 52, 7

Figer, D. F., McLean, I. S., \& Morris, M. 1999, ApJ, 514, 202

Frew, D. J. 2004, J. Astron. Data, 10, 6

van Genderen, A. M. 2001, A\&A, 366, 508

van Genderen, A. M., van Leeuwen, F., \& Brand, J. 1982, A\&AS, 47, 591

Golay, M. 1958, Publ. Obs. Geneve Ser. A, 57, 373

González, R. F., \& Cantó, J. 2008, A\&A, 477, 373

Gottlieb, E. W., \& Liller, W. 1978, ApJ, 225, 488

Groh, J. H., Damineli, A., \& Jablonski, F. 2007, A\&A, 465, 993

Groh, J. H., Hillier, D. J., \& Damineli, A. 2009a, ApJ, 698, 1698

Groh, J. H., Hillier, D. J., Damineli, A., et al. 2009b, ApJ, 705, L25

Groh, J. H., Hillier, D. J., \& Damineli, A. 2011, ApJ, 736, 46

Gvaramadze, V. V., Kniazev, A. Y., \& Fabrika, S. 2010, MNRAS, 405, 1047

Hammerschlag-Hensberge, G., Zuiderwijk, W. J., van den Heuvel, E. P. J., \& Hensberge, H. 1976, A\&A, 49, 321

Hammerschlag-Hensberge, G., Zuiderwijk, W. J., De Loore, C., \& van den Heuvel, E. P. J. 1979, A\&A, 76, 245

Hanson, M. M. 2003, ApJ, 597, 957

Hanson, M. M., Conti, P. S., \& Rieke, M. J. 1996, ApJS, 107, 281

Hanson, M. M., Rieke, G. H., \& Luhman K. L. 1998, AJ, 116, 1915 
Hanson, M. M., Kudritzki, R.-P., Kenworthy, M. A., Puls, J., \& Tokunaga, A. T. 2005, ApJS, 161, 154

Harries, T. J., \& Howarth, I. D. 1996, A\&AS, 119, 61

Harris, D. H., Woolf, N. J., \& Rieke, G. H. 1978, ApJ, 226, 829

Harries, T. J., Howarth, I. D., \& Evans, C. J. 2002, MNRAS, 337, 341

Heger, A., Langer, N., \& Woosley, S. E. 2000, ApJ, 528, 368

Hendry, M. A., Smartt, S. J., Skillman, E. D., et al. 2008, MNRAS, 388, 1127

Hill, G. M., Moffat, A. F. J., St-Louis, N., \& Bartzakos, P. 2000, MNRAS, 318, 402

Hillier, D. J., \& Miller, D. L. 1998, ApJ, 496, 407

Hillier, D. J., \& Miller, D. L. 1999, ApJ, 519, 354

Hillier, D. J., Crowther, P. A., Najarro, F., \& Fullerton, A. W. 1998, A\&A, 340, 483

Hiltner, W. A. 1956, ApJS, 2, 389

Hoffleit, D. 1933, Harvard Obs. Bull., 892, 19

Hubeny, I., \& Lanz, T. 1995, ApJ, 439, 875

Houck, J. R., Roellig, T. L., van Cleve, J., et al. 2004, ApJS, 154, 18

Humphreys, R., \& Davidson, K. 1979, ApJ, 232, 409

Humphreys, R., \& Davidson, K. 1994, PASP,106, 1025

Humphreys, R., Davidson, K., \& Smith, N. 1999, PASP, 111, 1124

Hutchings, J. B. 1968, MNRAS, 141, 219

Hutchings, J. B. 1970, MNRAS, 147, 161

Hutchings, J. B. 1976, PDAO, 14, 355

Hutchings, J. B. 1981, PASP, 93, 50

Jaschek, M., \& Jaschek, C. 1973, PASP, 85, 127

Jaschek, C., Jaschek, M., \& Kucewicz, B. 1964, ZA, 59, 108

Johnson, H. L. 1965, ApJ, 141, 923

Johnson, H. L., \& Borgman, J. 1963, BAN, 17, 115

Johnson, H. L., \& Morgan, W. W. 1953, ApJ, 117, 313

Johnson, H. L., Iriarte, B., Mitchell, R. I., \& Wisniewski, W. Z. 1966, Comm. Lunr Pan. Lab., 4, 99

Johnson, H. L. 1968, in Stars and Stellar Systems VII, ed. B. M. Middlehurst, \& L. A. Aller (Chicago: Univ. of Chicago Press), 167

Kaper, L., Lamers, H. J. G. L. M., Ruymaekers, E., van den Heuvel, E. P. J., \& Zuidervijk, E. J. 1995, A\&A, 300, 446

Kaper, L., van der Meer, A., \& Najarro, F. 2006, A\&A, 457, 595

Kastner, J. H., Buchanan, C. L., Sargent, B., \& Forrest W. J. 2006, ApJ, 638, L29

Kaufer, A., Stahl, O., Wolf, B., et al. 1996, A\&A, 305, 887

Kaufer A., Stahl, O., Wolf, B., et al. 1997, A\&A, 320, 273

Kaufer, A., Stahl., O., Prinja, R. K., \& Witherick, D. 2006, A\&A, 447, 325

Keenan, P. C. 1971, Contr. KPNO No. 554, 35

Kilkenny, D., can Wyk, F., Marang, F., et al. 1993, SAAOC, 15, 73

Kiminki, D. C., Kobulnicky, H. A., Kinemuchi, K., et al. 2007, ApJ, 664, 1102

Kiminki, D. C., Kobulnicky, H. A., Gilbert, I., Bird, S., \& Chunev, G. 2009, AJ, 137,4608

Klochkova, V. G., \& Chentsov, E. L. 2004, Astron. Rep., 48, 1005

Knödlseder, J. 2000, A\&A, 360, 539

Knoechel G., \& Moffat, A. F. J. 1982, A\&A, 110, 263

Kobulnicky, H. A., Molnar, L. A., \& Jones, T. J. 1994, AJ, 107, 1433

Koen, C., \& Eyer, L. 2002, MNRAS, 331, 45

Kruszewski, A. 1971, AJ, 76, 576

Kudritzki, R.-P. 2010, AN, 331, 459

Langer, N., Hamann, W.-R., Lennon, M., et al. 1994, A\&A, 290, 819

van Leeuwen, F., Evans, D. W., Grenon, M., et al. 1997, A\&A, 323, L61

van Leeuwen, F., van Genderen, A. M., \& Zegelaar, I. 1998, A\&AS, 128, 117

Lefèvre, L., Marchenko, S. V., Moffat, A. F. J., \& Acker, A. 2009, A\&A, 507 1141

Leitherer, C., \& Robert, C. 1991, ApJ, 377, 629

Leitherer, C., \& Wolf, B. 1984, A\&A, 132, 151

Leitherer, C., Hefele, H., Stahl, O., \& Wolf, B. 1982, A\&A, 108, 102

Leitherer, C., Stahl, O., Wolf, B., \& Bertout, C. 1984, A\&A, 140, 199

Lennon, D. J., Dufton, P. L., \& Fitzsimmons, A. 1992, A\&AS, 94, 569

Lenorzer, A., Vandenbussche, B., Morris, P., et al. 2002, A\&A, 384, 473

Lesh, J. R. 1968, ApJS, 17, 371

Liermann, A., Hamann, W.-R., \& Oskinova, L. M. 2009, A\&A, 494, 1166

Lopez, J. A., \& Walsh, J. R. 1984, Rev. Mex. Astron. Astrofis., 9, 3

Marco, A., \& Negueruela, I. 2009, A\&A, 493, 79

Markova, N., \& Puls, J. 2008, A\&A, 478, 823

Markova, N., \& Valchev, T. 2000, A\&A, 363, 995

Martins, F., \& Plez, B. 2006, A\&A, 457, 637

Martins, F., Schaerer, D., \& Hillier, D. J. 2005, A\&A, 436, 1049

Martins, F., Genzel, R., \& Hillier, D. J. 2007, A\&A, 468, 233

Martins, F., Hillier, D. J., Paumard, T., et al. 2008, A\&A, 478, 219

Massey, P., \& Thompson, A. B. 1991, AJ, 101, 1408

Mathewson, D. S., \& Ford, V. L. 1970, MemRAS, 74, 139

McLean, I. S. 1979, MNRAS, 186, 265

McLean, I. S., \& Clarke, D. 1979, MNRAS, 186, 245

McMillan, R. S., \& Tapia, S. 1977, ApJ, 212, 714
Merrill, P. W., \& Burwell, C. G. 1933, ApJ, 78, 87

Merrill, P. W., \& Burwell, C. G. 1943, ApJ, 98, 162

Merrill, P. W., Humason, M. L., \& Burwell, C. G. 1925, ApJ, 61, 389

Meynet, G., \& Maeder, A. 2005, A\&A, 429, 581

Miroshnichenko, A. S., Chentsov, E. L., \& Klochkova, V. G. 2000, A\&AS, 144, 379

Moffat, A. F. J., \& Fitzgerald, P. M. 1977, A\&A, 54, 263

Moffett, T. J., \& Barnes III, T. G. 1979, PASP, 91, 180

Moon, D.-S., Kaplan, D. L., Reach, W. T., et al. 2007, ApJ, 671, L53

Morel, T., Marchenko, S. V., Pati, A. K., et al. 2004, MNRAS, 351, 552

Morgan, W. W., Johnson, H. L., \& Roman, N. G. 1954, PASP, 66, 85

Morgan W. W., Code, A. D., \& Whitford, A. E. 1955, ApJS, 2, 41

Munari, U., Siviero, A., Bienaymé, O., et al. 2009, A\&A, 503, 511

Najarro, F. 2001, P Cygni 2000: 400 Years of Progress, ed. M. de Groot, \& C. Sterken (San Francisco, CA: ASP), ASP Conf. Ser., 233, 133

Najarro, F., Krabbe, A., Genzel, R., et al. 1997, A\&A, 325, 700

Najarro, F., Hillier, D. J., Figer, D., \& Geballe, T. R. 1999, in The Central Parsecs of the Galaxy, ed. H. Falcke et al. (San Francisco, CA: ASP), ASP Conf. Ser., 186,340

Najarro, F., Figer, D. F., Hillier, D. J., \& Kudritzki, R. P. 2004, ApJ, 611, L105

Najarro, F., Figer, D. F., Hillier, D. J., Geballe, T. R., \& Kudritzki, R. P. 2009, ApJ, 691, 1816

Negueruela, I., Marco, A., Herrero, A., \& Clark, J. S. 2008, A\&A, 487, 575

Negueruela, I., Clark, J. S., \& Ritchie, B. W. 2010, A\&A, 516, 78

Nicolet, B. 1978, A\&AS, 34, 1

Nordsieck, K. H., Wisniewski, J., Babler, B. L., et al. 2001, 400 Years of Progress, ed. M. de Groot, \& C. Sterken (San Francisco, CA: ASP), ASP Conf. Ser., 233, 261

Nordstrom, B. 1975, A\&AS, 21, 193

Parkes, G. E., Culhane, J. L., Mason, K. O., \& Murdin, P. G. 1980, MNRAS, 191, 547

Payne-Gaposchkin, C., \& Gaposchkin, S. 1938, Variable Stars (Cambridge, Mass.: Harvard College Observatory), 329

Percy, J. R., \& Welch, D. L. 1983, PASP, 95, 491

Percy, J. R., Palaniappan, R., Seneviratne, R., Adelman, S. J., \& Markova, N. 2008, PASP, 120, 311

Perry, C. L., Hill, G., \& Christodoulou, D. M. 1991, A\&AS, 90, 195

Perryman, M. A. C., Lindegren, L., \& Kovalevsky, J., et al. 1997, A\&A, 323, L49

Persi, P., \& Ferrari-Toniolo, M. 1982, A\&A, 112, 292

Petrovic, J., Langer, N., \& van der Hucht, K. A. 2005, A\&A, 435, 1013

Pickering, E. C., \& Fleming, W. P. 1896, ApJ, 4, 369

Rabod, D., Cramer, N., \& Bernasconi, P. A. 1997, A\&A, 325, 167

Rauw, G., De Becker, M., \& Linder, N. 2005, in Proc. Massive Stars and High-Energy Emission in OB Associations, a workshop of the JENAM 2005 Distant Worlds, held in Liège, Belgium, ed. G. Rauw, Y. Nazé, R. Blomme, \& E. Gosset (Liège), 103

Reed, B. C. 2003, AJ, 125, 2513

Reipurt, B. 2008, Handbook of star forming regions, Volume II: The southern sky ASP Monograph Publications, ed. B. Reipurth (San Francisco, CA: ASP), 5, 401

Rimmer, W. B. 1930, MmMtS, 2, 1

Ritchie, B. W., Clark, J. S., Negueruela, I., \& Najarro, F. 2009, A\&A, 507, 1597

Ritchie, B. W., Clark, J. S., Negueruela, I., \& Langer, N. 2010, A\&A, 520, A48

Rivinius, T., Stahl, O., Wolf, B., et al. 1997, A\&A, 318, 819

Robberto, M., \& Herbst, T. M. 1998, ApJ, 498, 400

Rosendhal, J. D. 1973, ApJ, 186, 909

Sana, H., Rauw, G., \& Gosset, E. 2001, A\&A, 370, 121

Sana, H., Gosset, E., Rauw, G., Sung, H., \& Vreux, J.-M. 2006a, A\&A, 454, 1047

Sana, H., Rauw, G., Nazeé, Y., Gosset, E., \& Vreux, J.-M. 2006b, MNRAS, 372, 661

Sana, H., Rauw, G., Sung, H., Gosset, E., \& Vreux, J.-M. 2007, MNRAS, 377, 945

Sana, H., Gosset, E., Nazé, Y., Rauw, G., \& Linder, N. 2008, MNRAS, 386, 447 Santolaya-Rey, A. E., Puls, J., \& Herrero, A. 1997, A\&A, 323, 488

Schaller, G., Schaerer, D., Meynet, G., \& Maeder, A. 1992, A\&AS, 96, 269

Schild, R. E., Hiltner, W. A., \& Sanduleak, N. 1969, ApJ, 156, 609

Schild, R. E., Neugebauer, G., \& Westphal, J. A. 1971, AJ. 76. 237

Schilt, J., \& Jackson, C. 1952, AJ, 56, 209

Schmidt, G. D., Elston, R., \& Lupie, O. L. 1992, AJ, 104, 1563

Schulte, D. H. 1958, ApJ, 128, 41

Schulz, A., \& Lenzen, R. 1983, A\&A, 121, 158

Scuderi, S., Panagia, N., Stanghellini, C., Trigilio, C., \& Umana, G. 1998, A\&A, 332,251

Searle, S. C., Prinja, R. K., Massa, D., \& Ryans, R. 2008, A\&A, 481, 777

Sharpless, S. 1957, PASP, 69, 239

Simón-Díaz, S., \& Herrero, A. 2007, A\&A, 468, 1063 
J. S. Clark et al.: On the nature of the galactic early-B hypergiants

Skrutskie, M. F., Cutri, R. M., Stiening, R., et al. 2006, AJ, 131, 1163

Slettebak, A. 1956, ApJ, 124, 173

Sloan, G. C., Kraemer, K. E., Price S. D., \& Shipman, R. F. 2003, ApJS, 147, 379

Smith, N., \& Conti, P. S. 2008, ApJ, 679, 1467

Sneden, C., Gerhz, R. D., Hackwell, J. A., York, D. G., \& Snow, T. P. 1978, ApJ, 223, 168

Souza, S. P., \& Lutz, B. Y. 1980, ApJ, 235, L87

Stahl, O., Kaufer, A., \& Tubbesing, S. 1999, in Optical and infrared spectroscopy of circumstellar matter, ed. E. W. Guenther, B. Stecklum, \& S. Klose (San Francisco, CA: ASP), ASP Conf. Ser., 188, 331

Stahl, O., Jankovics, I., Kovács, et al. 2001, A\&A, 375, 54

Stahl, O., Gang, T. Sterken, C., et al. 2003, A\&A, 400, 279

Stein, W. A., \& Gillettt, F. C. 1971, Nature, 233, 72

Sterken, C. 1977, A\&A, 57, 361

Sterken, C., \& Wolf, B. 1978, A\&A, 70, 641

Sterken, C., de Groot, M., \& van Genderen A. M. 1997, A\&A, 326, 640

Sterken, C., Arentoft, T., Duerbeck, H. W., \& Brogt, E. 1999, A\&A, 349, 532

Torres-Dodgen, A. V., Tapia, M., \& Carroll, M. 1991, MNRAS, 1991, 249, 1

Urbaneja, M. A., Herrero, A., Kudritzki, R.-P., et al. 2005, ApJ, 635, 311

van Breda, I. G., \& Whittet, D. C. B. 1977, Ap\&SS, 48, 297

Vidal, N. V. 1973, ApJ, 186, L81
Voelcker, K. 1975, A\&AS, 22, 1

Walborn, N. R. 1971, ApJS, 23, 257

Walborn, N. R. 1975, PASP, 87, 759

Walborn, N. R. 1980, ApJS, 44, 535

Walborn, N. R., \& Fitzpatrick, E. L. 1990, PASP, 102, 379

Walborn, N. R., Stahl, O., \& Gamen, R. C. 2008, ApJ, 683, L33

Waldron, W. L., Corcoran, M. F., Drake, S. A. \& Smale, A. P. 1998, ApJS, 118, 217

Wellstein, S., \& Langer, N. 1999, A\&A, 350, 148

Wendker, H. J., \& Altenhoff, W. J. 1980, A\&A, 92, L5

Westerlund, B. 1959, PASP, 71, 156

White, R. L., \& Becker, R. H. 1983, ApJ, 272, L19

Whittet, D. C. B., van Breda, I. G., \& Glass, I. S. 1976, MNRAS, 177, 625

Whittet, D. C. B., Martin, P. G., Hough, J. H., et al. 1992, ApJ, 386, 562

Whittet, D. C. B., Boogert A. C. A., Gerakines, P. A., et al. 1997, ApJ, 490, 729

Wisniewski, W. Z., Wing, R. F., Spinard, H., \& Johnson, H. L. 1967, ApJ, 148, L29

Wolf, B., \& Stahl, O. 1985, A\&A, 148, 412

Woźniak, P. R., Vestrand, W. T., Akerlof, C. W., et al. 2004, AJ, 127, 2436

Zappala, R. R. 1970, in Proc. Conference on Late Type Stars, ed. G. W. Lockwood, \& H. M. Dyck (Kitt Peark Contr. No. 554, Lick Obs. Bull. No. 610) 


\section{Appendix A: Summary of historical data for Galactic BHGs}

In order to understand the role of the BHG phase in the lifecycles of massive stars it is invaluable to contrast the long term (in)stability of such stars to other, potentially related evolutionary phases such as the LBVs. In order to accomplish this we undertook a literature review of all known Galactic examples. This has allowed the construction of the comprehensive spectroscopic and photometric datasets used in the quantitative modeling descibed in Sect. 4. To the best of our knowledge there are 16 known or candidate BHGs within the Galaxy, of which 8 have early (B1-4) spectral types. In constructing this list we have explicitly included 3 high luminosity stars of late-B spectral type which, although classified as luminosity class Iae, have prominent Balmer emission lines that appear characteristic of bona fide BHGs.

\section{A.1. The early-B HGs: Cyg OB2 \#12}

\section{A.1.1. Photometric variability}

Gottlieb \& Liller (1978) provide an extensive optical photometric history for Cyg OB2 \#12, comprising, where available, annual mean $B$ band magnitudes from $\sim 1890-1980$. They report low level annual variability $\left(m_{B} \sim 14.5-15.0\right.$ mag.) albeit with no apparent secular evolution. Despite being somewhat limited, the compilation of multi-epoch UBVRIJHK photometry presented in Table A.1 is consistent with little photometric activity being present ${ }^{9}$.

While systematic photometric monitoring of Cyg OB2 \#12 ceased after 1980 - fortuitously at a point when spectroscopic observations increased in frequency (Sect. A.1.2) - two further long-term photometric datasets are available. The first, acquired via the HipParcos mission (ESA 1997; Perryman et al. 1997), provides data from 1989 November to 1993 March, with the passband, $H_{\mathrm{p}}$, spanning 3400-9000 $\AA$ (van Leeuwen et al. 1997). Subsequently, the Northern Sky Variability Survey (NSVS; Woźniak et al. 2004) yielded a further year long dataset (1999 April-March 2000); this operated without filters, resulting in a wide optical band with a response defined by that of the CCD, extending from 450 to $1000 \mathrm{~nm}$.

These data are summarised in Fig. 3 and although the nonstandard passbands do not allow a direct comparison to previous data they do constrain variability over the periods in question. As with the results of Gottlieb \& Liller (1978), these datasets indicate that low level ( $\sim 0.3 \mathrm{mag})$ non secular and aperiodic variability is present, which occurs over rather short ( days) timescales.

While only single epoch mid-IR observations are available (Table A.2), multiple epochs of radio continuum observations are available in the literature, and are summarised in Table A.3. While the spectral indices derived from these data are consistent with thermal emission from a partially optically thick stellar wind, these fluxes demonstrate significant variability. As with the short period photometric variability, a possible interpretation of this behaviour is provided in Sect. 3 .

\footnotetext{
9 We note that in common with early observations of all the BHGs considered, interpretation is complicated by the lack of a reported date for some observations - a problem also present for the spectroscopic observations.
}

\section{A.1.2. Spectral classification and variability}

A summary of the multiwavelength spectroscopy obtained for Cyg OB2 \#12 over the past half century is presented in Table A.4. The spectral classifications given are those reported by the works in question; reclassification is provided only for those occasions where the source papers assumed an earlier result (this being the case for the IR studies, where Cyg OB2 \#12 was typically included as a spectral standard). Regarding reclassification, a luminosity class of $\mathrm{Ia}^{+}$is implictly assumed throughout the following discussion.

4-6000 Å: Souza \& Lutz (1980), Massey \& Thompson (1991), Klochkova \& Chentsov, (2004) and Kiminiki et al. (2007) all provide a detailed discussion of the classification of Cyg OB2 \#12 from 4000-5000 ^ spectra at high resolution and S/N, using the He I $4471 \AA: M g$ II $4481 \AA$ line ratio as the primary spectral-classification criterion. Following this methodology, spectral types in the range B3-B8 have been inferred by these authors, with variability in the line ratio (e.g. Kiminiki et al. 2007) taken as evidence for changes in the spectral type of Cyg OB2 \#12.

However, we caution that extreme care must be taken in inferring changes in the physical properties of the star from this diagnostic. Firstly, the $\mathrm{Mg}_{\text {II }} 4481 \AA$ A transition shows a dependence on stellar luminosity and so callibrations of spectral type versus the He I $4471 \AA$ A:Mg II $4481 \AA$ A line ratio derived from lower luminosity stars may not a priori be directly applied to Cyg OB2 \#12. More critically and as demonstrated in Sect. 4.1, this ratio is highly sensitive to the properties of the photosphere/wind transitional zone. Consequently, changes in the structure of the inner wind - which we may infer from line profile variability in $\mathrm{H} \alpha$ (see below) - at constant stellar temperature will cause this line ratio to vary, and in turn lead to the erroneous conclusion that because the spectral type has varied then the temperature has also changed.

Given the quality of their spectra - and in conjunction with data presented in Walborn \& Fitzpatrick (1990) - Kiminki et al. (2007) also employed the weak absorption feature at $\sim 4542 \AA$ as a classification criterion, which they attributed to a blend of He II and Fe II. While an absorption feature at $\sim 4542 \AA$ is observed in B1.5-4 supergiants, it is unlikely to be due to He II (seen only for B0 and earlier supergiants) or Fe II (B5 and later). Given these uncertainties, we instead choose to employ the Si III $4552 \AA / S i$ II $4128 \AA$ line ratio as a primary temperature diagnostic. Thus, upon consideration of the above, we suggest a spectral classification of B5 for the 1992 July 22 spectrum and B4 for the 1998 August, 2000 September and 2008 July spectra (Fig. A.1), noting that the marginally later classification in 1992 is likely to be a result of the lower $\mathrm{S} / \mathrm{N}$ and spectral resolution of these data. We thus find no evidence for changes in the spectral type of Cyg OB2 \#12 in these data.

6-8000 A: the spectral region surrounding $\mathrm{H} \alpha$ is largely devoid of classification criteria (e.g. Negueruela et al. 2010). However (i) the strong central $\mathrm{H} \alpha$ emission peak superimposed on broad emission wings; (ii) lack of He II 6527, $6683 \AA$ absorption; (iii) absence of $\mathrm{S}_{\text {IV }} 6668,6701 \AA$ emission; and (iv) the presence of strong $\mathrm{C}_{\text {II }} 6578,6582 \AA$ photospheric lines are 
uniformly present in spectra from 1992 onwards $^{10}$ and are all consistent with a highly luminous B supergiant (Fig. 1). As found by Klochkova \& Chentsov (2004), the central peak of the $\mathrm{H} \alpha$ profile appears to be variable at low projected velocities $\left(\leq 140 \mathrm{~km} \mathrm{~s}^{-1}\right)$.

8-9000 ̊̊: despite spanning a period of over 16 years, the $3 I$ band spectra are essentially identical (a representative spectrum from 2008 is presented in Fig. A.2). To classify Cyg OB2 \#12 from these data we employed the scheme described in Negueruela et al. (2010), supplemented by spectra of other early-mid BSGs and BHGs of known spectral type. While the BHG spectra show a similar morphological progression to those of normal BSGs (Negueruela et al. 2010, Fig. A.2), there is no indication of emission in the Paschen series (noting that the lower Balmer transitions are seen in emission). While such a finding precludes the separation of BHGs from BSGs in the spectroscopic window in which GAIA - the Global Astrometric Interferometer for Astrophysics - will operate, the lack of wind contamination aids in the quantitative determination of the underlying stellar parameters (Sect. 4).

Regarding the classification of Cyg OB2 \#12, the presence of $\mathrm{N}_{\mathrm{I}}$ absorption lines indicates a spectral type of B3 or later, with their comparative weakness favouring B3. This finding is consistent with both the strength of the O I $8446 \AA$ line, which clearly lies between B2.5Ia and B4Ia (see Fig. A.2), and the temperature sensitive $\mathrm{Pa} 15 / \mathrm{Pa} 11$ and $\mathrm{Pa} 16 / \mathrm{Pa} 11$ line ratios. However, the differences between B3 and B4 Ia stars are small - hence we suspect that the slight discrepancies between classifications based on the $\sim 4-5000 \AA$ and $\sim 8-9000 \AA$ windows evident for data obtained in 1992 and 2008 are unlikely to be real. Likewise, distinguishing between B5 and B8 supergiants on the basis of $I$ band spectra alone is particularly difficult (Negueruela et al. 2010); hence we caution against using the results of Sharpless (1957) and Zappala (1970) as evidence for spectroscopic variability prior to 1970 . Echoing Sect. 4, changes in the physical properties of the photosphere/wind transitional zone have negligible impact on the $I$ band spectral morphology - hence explaining potential discrepancies between the spectral type of Cyg OB2 \#12 determined from the $I$ band and the He I $4471 \AA$ ̊:Mg II $4481 \AA$ diagnostic.

0.98-1.10 $\mu \mathrm{m}$ : two epochs of observations from 1990 and 1992 are presented by Conti \& Howarth (1999), who report no variability between the spectra. Unfortunately a paucity of spectral features and little calibration data complicate classification from these data, although the presence of weak He I $1.031 \mu \mathrm{m}$ suggests a classification of $\mathrm{B} 8 \mathrm{Ia}^{+}$or earlier and the absence of He II $1.0124 \mu \mathrm{m}$ provides an upper limit of B3 $\mathrm{Ia}^{+}$. Such a result is consistent with that derived from our spectra obtained on 1992 July 22.

1.66-1.72 $\mu \mathrm{m}$ : a single epoch of $\mathrm{H}$ band spectroscopy was presented by Hanson et al. (1998), with Br11 and He I $1.700 \mu \mathrm{m}$ lines in absorption and of equal strength and He II $1.693 \mu \mathrm{m}$

\footnotetext{
10 The low resolution and $\mathrm{S} / \mathrm{N}$ of previously published spectra are insufficient to comment on the presence, or otherwise, of photospheric features.
}

absent. The lack of He II $1.693 \mu \mathrm{m}$ is characteristic of a B spectral type, with $E W(\mathrm{Br} 11) \geq E W(\mathrm{He} \mathrm{I})$ occuring for $\mathrm{B} 1.5 \mathrm{Ia}^{+}$ and later (at the resln. of the spectrum in question) and only becoming $\gg 1$ at $\mathrm{B} 8 \mathrm{Ia}^{+}$, hence providing upper and lower limits to the spectral type (Hanson et al. 1998; 2005).

2.0-2.4 $\mu \mathrm{m}$ : Hanson et al. (1996) present a $K$ band spectrum from 1994, in which $\mathrm{Br} \gamma$ and $\mathrm{He}_{\mathrm{I}} 2.112 \mu \mathrm{m}$ are seen in absorption. For a star of the expected luminosity of Cyg OB2 \#12 and with the low $\mathrm{S} / \mathrm{N}$ and resolution of the data in question, only broad constraints are possible, with the lack of He II $2.189 \mu \mathrm{m}$ and the presence of He I $2.112 \mu \mathrm{m}$ implying a classification between B0-9 Ia.

2.35-4.10 $\mu \mathrm{m}$ : finally, Lenorzer et al. (2002) and Whittet et al. (1997) present $2.35-4.1 \mu \mathrm{m}$ spectra, which we may supplement with an additional spectrum from 1996 April 4. All four display emission in $\operatorname{Br} \alpha, \operatorname{Pf} \alpha$ and $\beta$, with the higher transitions seen in absoprtion. No variability is apparent between any of these spectra; hence we do not reproduce them here. Unfortunately, as with the $\sim 1-2 \mu \mathrm{m}$ window, there is a dearth of classification features in this region and we may only infer a spectral type between $\mathrm{B} 3-9 \mathrm{Ia}^{+}$from the presence and strength of the Brackett and Pfund series and the lack of $\mathrm{He}$ I photospheric lines.

To summarise - we find that the available data provides no substantive evidence for the long-term evolution of the spectral type of Cyg OB2 \#12 over the past half century, although this conclusion is necessarily weaker prior to the $1990 \mathrm{~s}$, given the sparser dataset available. Nevertheless, this finding is entirely consistent with the long-term lightcurve. A classification of B3-4 $\mathrm{Ia}^{+}$appears appropiate thoughout this period, although short term variability is present in both wind (e.g. $\mathrm{H} \alpha \ldots \delta$ ) and photospheric lines (e.g. Kiminki et al. 2007).

Both phenomena are common in other luminous B super-/ hypergiants (e.g. Clark et al. 2010) where they are assumed to be due to, respectively, time-dependent wind structure and photospheric pulsations resulting in changes in photospheric temperature. Regarding the latter, changes in the He I $4471 \AA: \mathrm{Mg}_{\text {II }} 4481 \AA$ flux ratio have led to the conclusion that the spectral type is variable $\left(\mathrm{B} 3-8 \mathrm{Ia}^{+}\right)$, from which changes in the photospheric temperature $(16-12 \mathrm{kK})$ of Cyg OB2 \#12 have been inferred; such a range is fully consistent with models of the pulsating B supergiant HD 64760 (Kaufer et al. 2006) and the BHGs Wd1-7 and 42 (Clark et al. 2010). However we caution that the detailed non-LTE model atmosphere analysis reveals that variation in the physical structure of the photosphere/wind transitional zone can also lead to this behaviour; hence concluding that the photospheric temperature has been variable based on the current spectroscopic dataset is clearly premature.

\section{A.2. The early-B HGs: $\zeta^{1}$ Sco}

\section{A.2.1. Photometric variability}

Sterken (1977) and Sterken et al. (1997) have previously undertaken long-term(differential) uvby Stromgren and $V$ BLUW photometric campaigns between 1973-1974 and 1982-1995 respectively. They report low level ( $\Delta m \sim 0.01 \mathrm{mag})$ quasi-periodic variability over a wide range of timescales ( 16.5- 2000 days), with additional stochastic variability 
$(\Delta m \sim 0.05 \mathrm{mag})$ superimposed. (Aperiodic) low level $(\Delta m=$ $0.08 \mathrm{mag})$ photometric variations were also present in the Hipparcos dataset between 1989-1993 (Lefèvre et al. 2009) and between 1979-1980 (Burki et al. 1982).

In addition to these data Sterken et al. (1997) also present historical observations from the 10th-19th Century, to which we may add a number of more recent data from the 20th Century (Table A.1). Sterken et al. (1997) suggest that $\zeta^{1}$ Sco is a longterm variable, reporting $m_{V} \sim 2.8$ in the middle of the 18th Century, $m_{V} \sim 4.3$ and 5.4 in 1875 and 1878 and $m_{V} \sim 4.5-4.8$ between 1890-1900. The latter values are broadly consistent with the photometric data presented in Table A.1, which span the 20th Century and provide no evidence for the long term secular variability that characterises e.g. the LBV phase. Moreover, these data also demonstrate that the near-IR continuum is similarly stable, albeit over a shorter, $\sim 30 \mathrm{yr}$ baseline. We therefore conclude that the sole evidence for significant $\left(\Delta m_{V}>0.3 \mathrm{mag}\right)$ variability is provided by historical visual estimates dating from before $\sim 1890$, for which we are unfortunately unable to quantify the observational uncertainty.

Finally, we turn to the radio data, for which Bieging et al. (1989) report possible variability. This mirrors the findings for Cyg OB2 \#12 and we provisionally associate this behaviour with the same physical cause (Sect. 3).

\section{A.2.2. Spectroscopic classification and variability}

Given the brightness of $\zeta^{1}$ Sco, spectroscopic observations dating from the turn of the 19 th Century are reported in the literature, although a lack of accurate dates for the earlier observations complicates their interpretation. Nevertheless, a summary of these observations are presented in Table A.5; we note that the lower reddening to $\zeta^{1}$ Sco facilitates a more homgeneous dataset of blue end spectroscopy in comparison to Cyg OB2 \#12. Fortuitously, the period between 1900-1950 is well sampled and hence complements the sparse photometric dataset in this period.

This compilation reveals an absence of long-term variability or secular evolution of spectral type over a $\sim 110 \mathrm{yr}$ interval. The description of the main features of the optical spectra over this whole period are remarkably consistent; the spectra presented in Fig. A.1 for the period 1994-2009 demonstrating this stability. Nevertheless, numerous authors report line profile variability in the wind dominated $\mathrm{P}$ Cygni profile of $\mathrm{H} \alpha$ and $\beta$, with the higher $\mathrm{H}_{\mathrm{I}}$ transitions repeatedly varying between emission and absorption over at least a $\sim 50 \mathrm{yr}$ interval (cf. Table A.5 and refs. therein). Rivinius et al. (1997) report the rapid variability of both photospheric and wind lines between 1992-1995; we consider it likely that the variability observed in the $\mathrm{H} \alpha$ P Cygni line between 1998-2009 (Fig. 1) reflects a continuation of this behaviour.

\section{A.3. The early-B HGs: HD 190603}

Unfortunately, photometric datasets for HD 190603 are somewhat sparse in comparison to the previous two stars, with no photometric observations reported over the last decade and a $>16$ yr gap in the 1980 s-1990s (Table A.1). Nevertheless, we find no evidence for secular variability over the $\sim 46 \mathrm{yr}$ period from 1952-1998. Conversely, rapid photometric variability has been reported on several occassions by both Percy \& Welch (1983) and Koen \& Eyer (2002).

Likewise comparatively few spectral observations have been reported, a problem exacerbated by the lack of reduced spectra being presented. Consequently we are forced to simply present the spectral classifications reported for HD 190603 in Table A.6. These data indicate a corresponding lack of spectroscopic evolution over a 50 year period. Indeed, the description of the spectrum by both Beals (1946) and Merrill \& Birwell (1943) as, respectively, that of a P Cygni supergiant and a Be star, suggest a similar morphology prior to 1933. The latter authors further described variable emission lines; similar findings being reported by Rosendhal (1973) and Rivinius et al. (1997).

\section{A.4. The early-B HGs: HD 80077, HD 169454 and BP Cru}

We next turn to the remaining early-B HGs. We refrain from tabulating their sparse photometric datasets but note that there is no evidence for secular variability in any of the three stars; for instance comparison of the optical (Hiltner 1956; Kilkenny et al. 1993) and near-IR (Whittet et al. 1997; Skrutskie et al. 2006) data for HD 169454 reveal constancy over $\sim 35$ and $\sim 26$ yr intervals respectively. However, following the previous discussions, rapid, low amplitude photometric variability appears ubiquitous (van Leeuwen et al. 1998; Sterken 1977 and HammerschlagHensberge et al. 1979, respectively).

As with HD 190603, Table A.6 summarises their spectral types as reported in the literature. No evidence for the long-term (secular) evolution of spectral type over timescales in excess of 30 yrs was found, with the description of HD 169454 as a B star with H I P Cygni emission lines (Merrill \& Burwell 1943) suggesting spectral stability for nearly a century. In contrast line profile variability (LPV) on a timescale of days-weeks is present in all 3 stars (Knoechel \& Moffat 1982; Rivinius et al. 1997; Kaper et al. 2006, respectively).

\section{A.5. The early-BHGs/WNVL stars Wd1-5 and 13}

The remaining early-B HGs are found within the massive young cluster $\mathrm{Wd} 1$, and on the basis of a restricted wavelength range (5800-8900 ^) were classified as borderline BHG/very late WN stars, forming an evolutionary sequence from Wd1-5 through -13 to the WN9h star Wd1-44 (Clark et al. 2008). Unfortuntely, these stars have only been observed over the past decade, but no evidence for long term variability has been found (Clark et al. 2010). However, Wd1-13 is a confirmed 9.27 day massive binary, while pronounced LPV in Wd1-44 also argues for such an identification. Consequently, we suspect that all three stars to have formed as the result of close binary evolution.

\section{A.6. The late-B HGs}

Finally, we examine the eight BHGs with spectral types of B5 and later that have been identified within the Galaxy. Three W7, W33 (both B5 $\mathrm{Ia}^{+}$) and W42a $\left(\mathrm{B} 9 \mathrm{Ia}^{+}\right)$- are located within the massive young cluster $\mathrm{Wd} 1$ and are discussed in detail in Clark et al. (2010). Unfortunately, the long-term spectroscopic and photometric datasets for these stars are less complete than those of the early-B HGs described above, although they are sufficient to confirm the presence of rapid LPV. This behaviour, as well as short term low amplitude photometric pulsations is also present in the other examples; HD 160529 (A9-B8 $\mathrm{Ia}^{+}$; Stahl et al. 2003), HD 168607 (B9 Ia ${ }^{+}$Chentsov et al. 2003, Sterken et al. 1999), HD 168625 (B8 Ia ${ }^{+}$; Sterken et al. 1999; Chentsov et al. 2003), HD 183143 (B7 Iae; Adelman et al. 2000; Chentsov et al. 2003) and HD 199478 (B8 Iae; Percy et al. 2008; Markova \& Valchev 2000), suggesting that 
the $\alpha$ Cygni instabilities are ubiquitous across the complete temperature and luminosity range spanned by BHGs.

This phenomenon is also found to extend to cooler temperatures, having been identified in a number of early-A (A0-2.5 $\mathrm{Ia}^{+} / \mathrm{Iae}$ ) stars with similar spectral morphologies to the BHGs - e.g. HD 92207 (A0 Iae; Sterken 1977; Kaufer et al. 1997), HD 223385 (A2.5 Ia+; Adelman \& Albayrak 1997; Chentsov 2003) and AS 314 (A0 Ia ${ }^{+}$; Miroshnichenko 2000).

However, unlike the early-B HGs, HD 160529 and 168607 demonstrate characteristic LBV photometric modulation, with the former also exhibiting correlated spectral type variability, while both HD 168625 and HD 92207 show evidence for a complex, dusty circumstellar environment (Roberto \& Herbst 1998; Clarke et al. 2005) implying recent enhanced mass loss, possibly associated with an LBV phase. 
A\&A 541, A145 (2012)
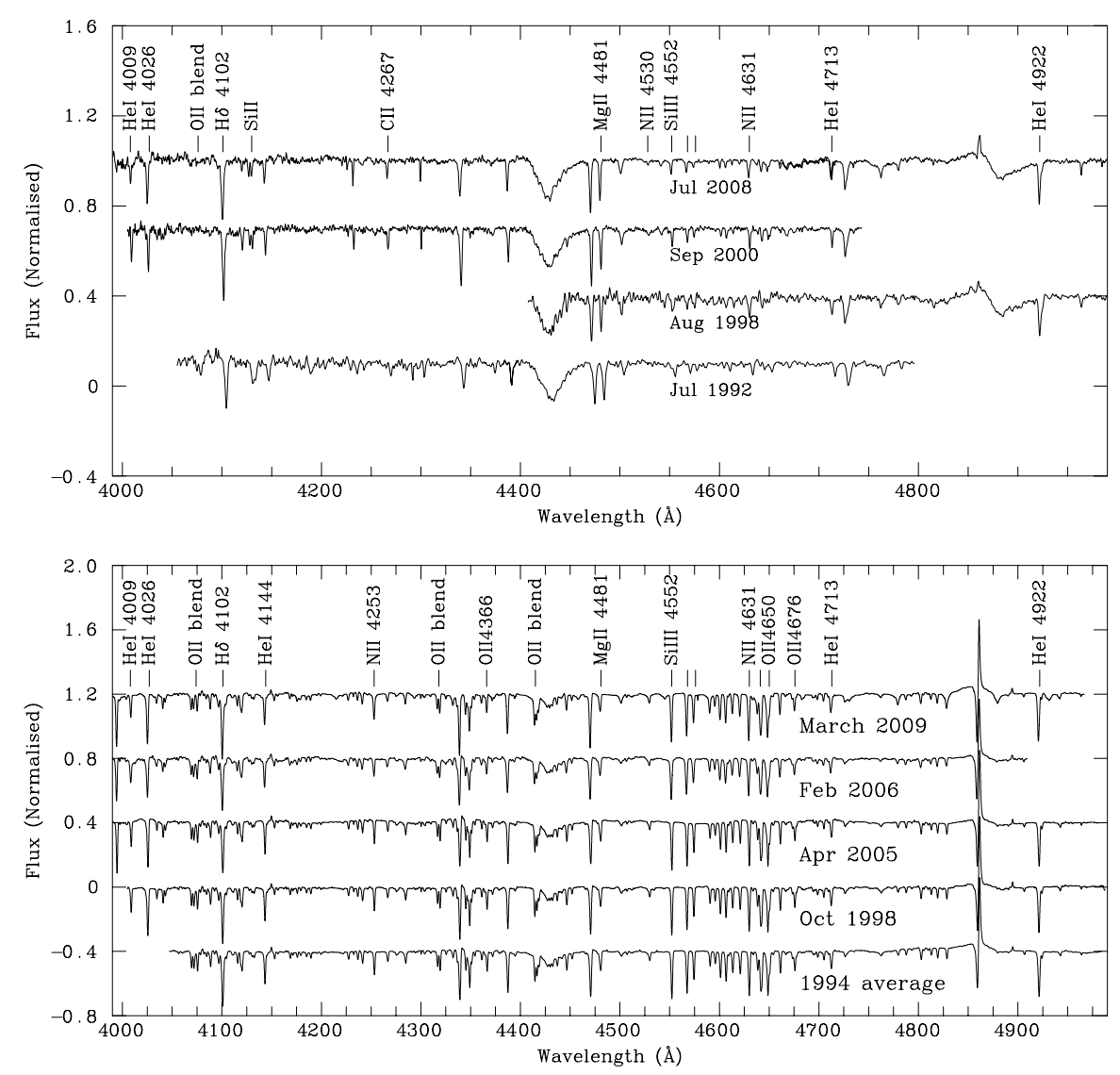

Fig. A.1. Long-term evolution of Cyg OB2 \#12 (1992-2008; top panel) and $\zeta^{1}$ Sco (1998-2009; bottom panel) in the $\sim 4000-4800 \AA$ spectral region, encompassing the Si III $4552 \AA / S i$ II $4128 \AA$ temperature diagnostic.

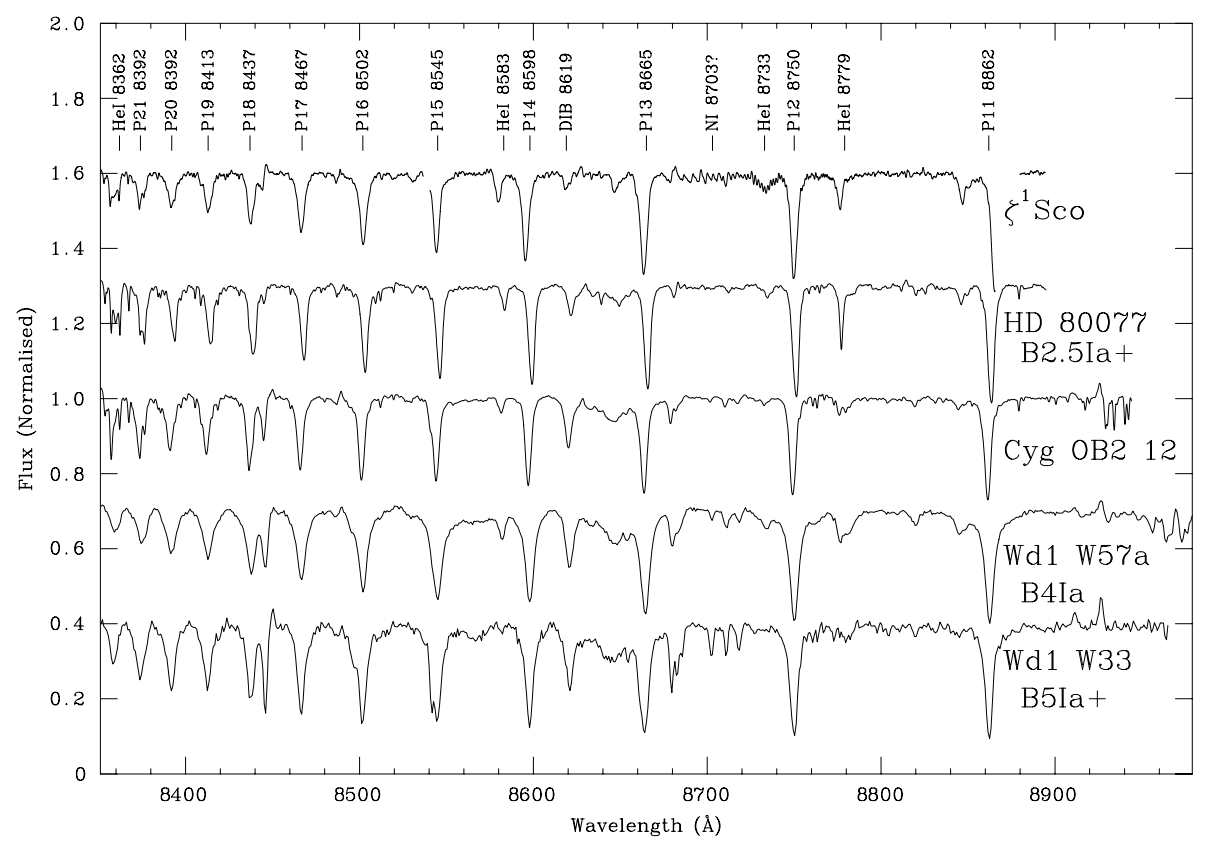

Fig. A.2. Comparison of the $I$ band spectra of Cyg OB2 \#12 and $\zeta^{1}$ Sco with other galactic BHGs. Note the systematic weakening of $\mathrm{He}_{\mathrm{I}}$ and strengthening of $\mathrm{N}_{\mathrm{I}}$ transitions with decreasing temperature, which permit spectral classification in whis wavelength range. 


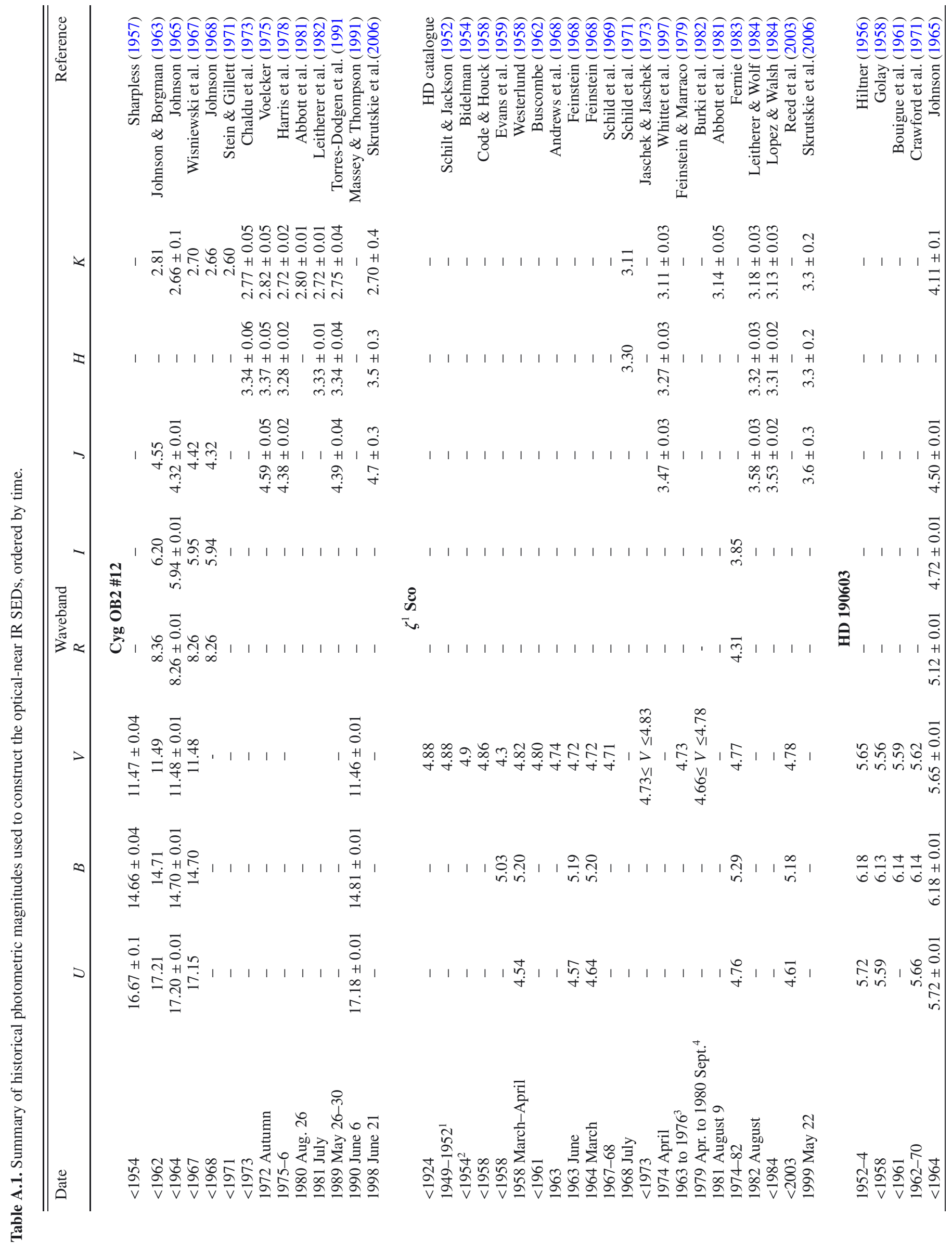




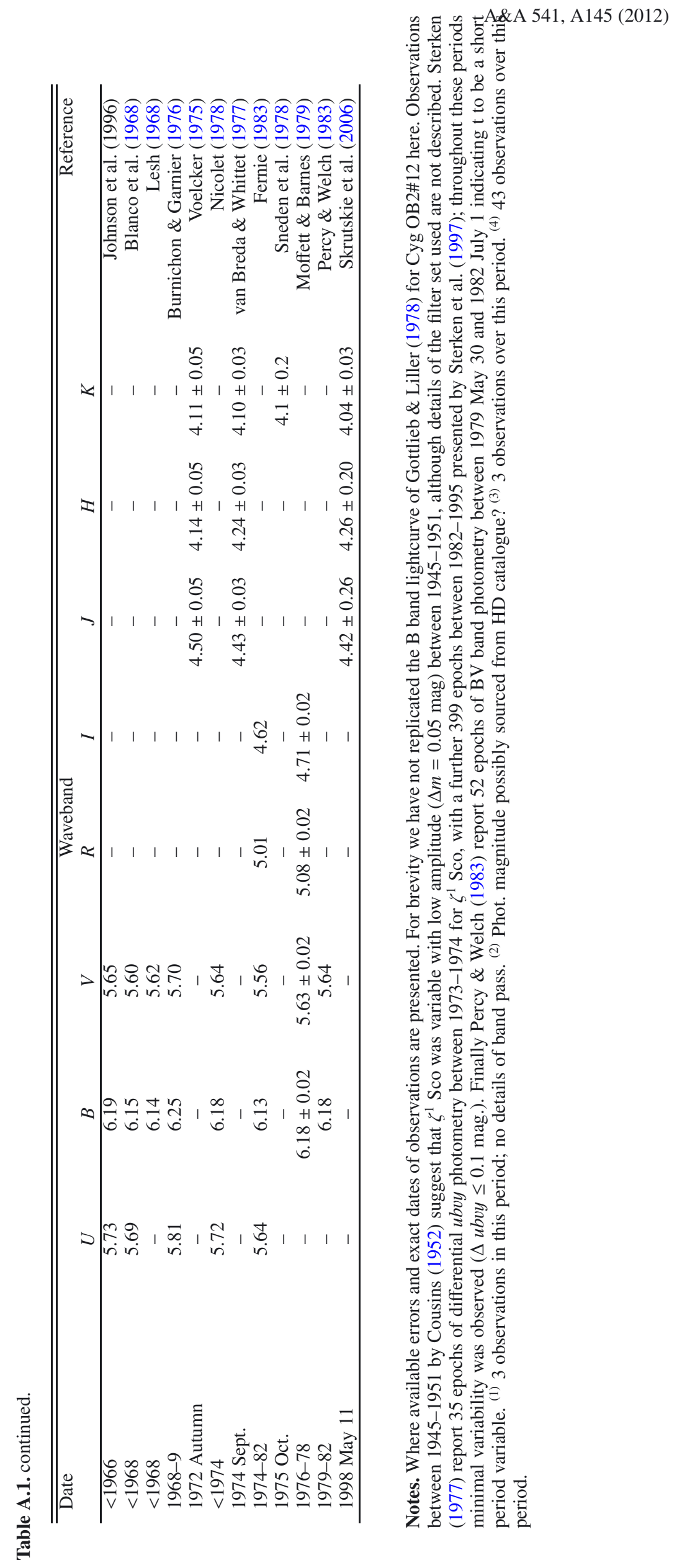

A145, page 26 of 36 
Table A.2. Summary of mid- to far-IR fluxes used to construct the SEDs.

\begin{tabular}{|c|c|c|}
\hline $\begin{array}{l}\text { Wavelength } \\
(\mu \mathrm{m})\end{array}$ & Magnitude & Reference \\
\hline \multicolumn{3}{|c|}{ Cyg OB2 \#12 } \\
\hline 2.30 & $2.72 \pm 0.01$ & Leitherer et al. (1982) \\
\hline 2.345 & $2.635 \pm 0.02$ & Harris et al. (1978) \\
\hline 3.013 & $2.405 \pm 0.02$ & Harris et al. (1978) \\
\hline 3.108 & $2.375 \pm 0.02$ & Harris et al. (1978) \\
\hline 3.420 & $2.281 \pm 0.02$ & Harris et al. (1978) \\
\hline 3.45 & $2.37 \pm 0.05$ & Abbott et al. (1981) \\
\hline 3.50 & $2.28 \pm 0.05$ & Chaldu et al. (1973) \\
\hline 3.57 & $2.28 \pm 0.02$ & Leitherer et al. (1982) \\
\hline 3.57 & $2.170 \pm 0.05$ & Torres-Dodgen et al. (1991) \\
\hline 3.58 & $2.217 \pm 0.02$ & Harris et al. (1978) \\
\hline 3.820 & $2.178 \pm 0.02$ & Harris et al. (1978) \\
\hline 4.29 & $2.057 \pm 0.118$ & MSX (Egan et al. 2001) \\
\hline 4.80 & $2.37 \pm 0.10$ & Abbott et al. (1981) \\
\hline 4.97 & $2.06 \pm 0.02$ & Leitherer et al. (1982) \\
\hline 8.28 & $1.946 \pm 0.044$ & MSX (Egan et al. 2001) \\
\hline 10.2 & $2.03 \pm 0.10$ & Abbott et al. (1981) \\
\hline 10.9 & $1.95 \pm 0.07$ & Leitherer et al. (1982) \\
\hline 12.0 & $1.642 \pm 0.00$ & IRAS PSC (1985) \\
\hline 12.13 & $1.900 \pm 0.056$ & MSX (Egan et al. 2001) \\
\hline 14.65 & $1.713 \pm 0.067$ & MSX (Egan et al. 2001) \\
\hline 20.0 & $1.54 \pm 0.10$ & Abbott et al. (1981) \\
\hline 21.34 & $1.575 \pm 0.079$ & MSX (Egan et al. 2001) \\
\hline 25.0 & 1.204 & IRAS PSC (1985) \\
\hline \multicolumn{3}{|c|}{$\zeta^{1}$ Sco } \\
\hline 3.45(L) & $2.98 \pm 0.03$ & Leitherer \& Wolf (1984) \\
\hline $4.7(\mathrm{M})$ & $2.90 \pm 0.03$ & Leitherer \& Wolf (1984) \\
\hline 8.28 & 2.624 & MSX (Egan et al. 2001) \\
\hline 12.13 & 3.416 & MSX (Egan et al. 2001) \\
\hline 14.65 & 3.560 & MSX (Egan et al. 2001) \\
\hline \multicolumn{3}{|c|}{ HD 190603} \\
\hline 3.6 & $3.96 \pm 0.18$ & Sneden et al. (1978) \\
\hline 4.9 & $3.89 \pm 0.20$ & Sneden et al. (1978) \\
\hline 8.28 & $3.68 \pm 0.04$ & MSX (Egan et al. 2001) \\
\hline 9.0 & $3.12 \pm 0.01$ & AKARI \\
\hline 12.13 & $3.47 \pm 0.08$ & MSX (Egan et al. 2001) \\
\hline 14.65 & $3.46 \pm 0.09$ & MSX (Egan et al. 2001) \\
\hline
\end{tabular}




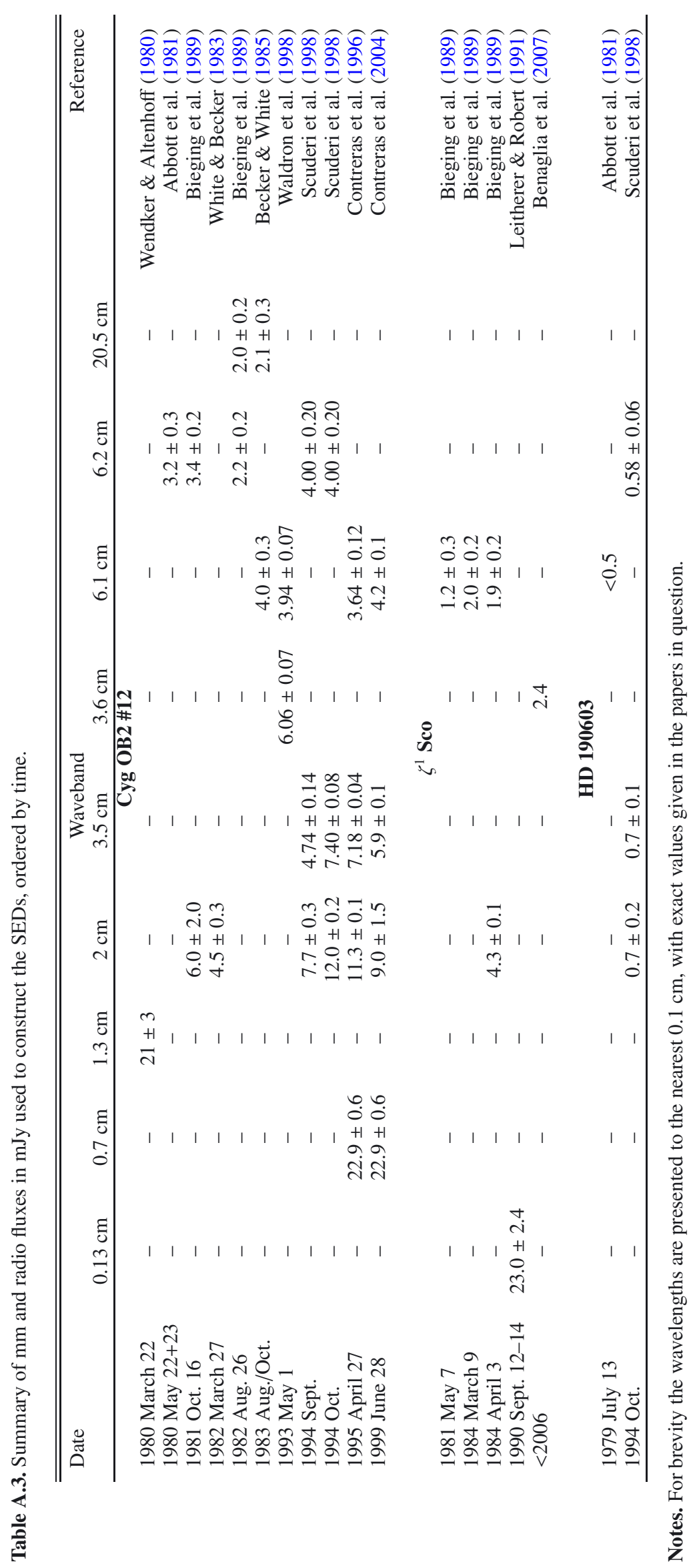


J. S. Clark et al.: On the nature of the galactic early-B hypergiants

Table A.4. Summmary of historical spectral observations of Cyg OB2 \#12.

\begin{tabular}{|c|c|c|c|c|}
\hline Date & Wavelength & Spectral type & Comments & Reference \\
\hline$\leq 1954$ & $4100-6600 \AA$ & $<A O I$ & Ho in emission & Morgan et al. (1954) \\
\hline$\leq 1957$ & 8-9000 ̊ & B5 Ia & - & Sharpless (1957) \\
\hline$\leq 1970$ & near IR & $B 8 I a^{+}$ & - & Zappala (1970) \\
\hline$\leq 1971$ & $?$ & - & Ho in emission & Bromage (1971) \\
\hline$\leq 1973$ & $\begin{array}{l}5-8700 \AA ̊+ \\
8-11000 \AA\end{array}$ & - & $\mathrm{H} \alpha$ in emission & Chaldu et al. (1973) \\
\hline 1977 July & $\begin{array}{c}3840-4480 \AA \\
+H \alpha\end{array}$ & $\mathrm{B} 8 \mathrm{Ia}^{+}$ & $\begin{array}{l}\text { Variable } \mathrm{H} \alpha \\
\text { in emission }\end{array}$ & Souza \& Lutz (1980) \\
\hline 1980 Autumn & $H \alpha$ & - & H $\alpha$ in emission & Leitherer et al. (1982) \\
\hline$\leq 1981$ & $4000-6600 \AA$ & - & $H \alpha+H \beta$ in emission & Hutchings (1981) \\
\hline $\begin{array}{l}\text { 1989-90 summer + } \\
\text { "few years earlier" }\end{array}$ & $\begin{array}{l}\text { encompasses } H \alpha \\
+\sim 4089-4686 \AA\end{array}$ & $\begin{array}{l}\text { B5 Ia } \\
\text { B5 Ia }\end{array}$ & $\begin{array}{c}\mathrm{H} \alpha+\mathrm{H} \beta \text { in emission, } \\
\mathrm{H} \gamma+\mathrm{H} \delta \text { infilled }\end{array}$ & Massey \& Thompson (1991) \\
\hline 1990 August & $0.98-1.10 \mu \mathrm{m}$ & $>$ B3 Ia,$<$ B8 Ia & - & Conti \& Howarth (1999) \\
\hline 1992 July & $0.98-1.10 \mu \mathrm{m}$ & $>$ B3 Ia, < B8 Ia & - & Conti \& Howarth (1999) \\
\hline 1992 July 17 & $\begin{array}{l}4036-4836 \AA \\
5765-9586 \AA\end{array}$ & $\begin{array}{c}\text { B4-5 Ia } \\
\text { B3 Ia }\end{array}$ & $\begin{array}{c}\mathbf{H} \gamma+\mathbf{H} \delta \text { in absorption } \\
\mathbf{H} \alpha \text { in emission }\end{array}$ & This work \\
\hline 1994 Sept. & $2-2.2 \mu \mathrm{m}$ & $>$ B0.5 Ia, $<$ B9 Ia & - & Hanson et al. (1996 ) \\
\hline 1995 July 17 & $6366-6772 \AA$ & - & $\mathrm{H} \alpha$ in emission & This work \\
\hline 1995 Dec. 23 & $2.35-8 \mu \mathrm{m}$ & $>$ B3 Ia, < B9 Ia & $\operatorname{Br} \alpha, \operatorname{Pf} \alpha+\beta$ in emission & Whittet et al. (1997) \\
\hline 1996 April 4 & $2.35-8 \mu \mathrm{m}$ & $>$ B3 Ia, < B9 Ia & $\operatorname{Br} \alpha, \operatorname{Pf} \alpha+\beta$ in emission & This work \\
\hline 1996 Oct. 17 & $2.35-8 \mu \mathrm{m}$ & > B3 Ia, < B9 Ia & $\operatorname{Br} \alpha, \operatorname{Pf} \alpha+\beta$ in emission & Whittet et al. (1997) \\
\hline 1997 June & $1.66-1.72 \mu \mathrm{m}$ & $>$ B1.5 Ia, $<$ B8 Ia & - & Hanson et al. (1998) \\
\hline 1998 Apr.-May & $2.35-8 \mu \mathrm{m}$ & $>$ B3 Ia, < B9 Ia & $\operatorname{Br} \alpha, \operatorname{Pf} \alpha+\beta$ in emission & Lenorzer et al. (2002) \\
\hline 1998 Aug. 09 & $\begin{array}{l}3940-5737 \AA \\
6366-6772 \AA\end{array}$ & $\begin{array}{c}\text { B4 Ia } \\
-\end{array}$ & $\begin{array}{l}\mathrm{H} \beta \text { in emission } \\
\mathrm{H} \alpha \text { in emission }\end{array}$ & This work \\
\hline 1999 July 10 & $5500-7700 \AA$ & B Ia & $\mathrm{H} \alpha$ in emission & Kiminki (2010, priv. comm.) \\
\hline 2000 July 10 & $5500-7700 \AA$ & B Ia & $\mathrm{H} \alpha$ in emission & Kiminki (2010, priv. comm.) \\
\hline 2000 Sept. 18 & $3600-5200 \AA$ & B3 Iae & $\mathrm{H} \beta$ infilled & Kiminki et al. (2007) \\
\hline 2000 Sept. 23 & $\begin{array}{l}4000-4750 \AA \\
6340-6740 \AA\end{array}$ & $\begin{array}{c}\text { B4 Ia } \\
-\end{array}$ & $\begin{array}{c}\mathbf{H} \gamma+\mathbf{H} \delta \text { in absorption } \\
\mathbf{H} \alpha \text { in emission }\end{array}$ & This work \\
\hline 2001 June 21 & $4542-7939 \AA$ & B5 Ia & Balmer line emission & Klochkova \& Chenstov (2004) \\
\hline 2001 August 24 & $3800-4500 \AA$ & B6 Iae & $\mathrm{H} \gamma+\mathrm{H} \delta$ in absorption & Kiminki et al. (2007) \\
\hline 2001 Sept. 01 & $3800-4500 \AA$ & B8 Iae & $\mathrm{H} \gamma+\mathrm{H} \delta$ in absorption & Kiminki et al. (2007) \\
\hline 2003 April 12 & 5273-6764 ̊ & B5 Ia & $\mathrm{H} \alpha$ emission & Klochkova \& Chenstov (2004) \\
\hline 2007 Aug. 22 & $7600-8900 \AA$ & B3 Ia & - & This work \\
\hline 2007 Aug. 30 & $5600-6800 \AA$ & B Ia & $\mathrm{H} \alpha$ in emission & Kiminki (2010, priv. comm.) \\
\hline 2008 July 22 & $\begin{array}{l}4000-5270 \AA \\
6450-7150 \AA \\
8350-8900 \AA\end{array}$ & $\begin{array}{c}\text { B4 Ia } \\
- \\
\text { B3 Ia }\end{array}$ & $\begin{array}{c}\mathrm{H} \beta \text { in emission } \\
\mathbf{H} \gamma+\mathbf{H} \delta \text { in absorption } \\
\mathbf{H} \alpha \text { in emission }\end{array}$ & This work \\
\hline
\end{tabular}

Notes. A parameter is listed in italics if not explicitly given (wavelength of observation) or the data from which it is derived was not presented. Parameters given in bold are from this work. $\mathrm{H} \alpha$ is variable between the two epochs of observations presented by Klochkova \& Chenstov (2004) as $\mathrm{H} \beta$ and $\gamma$ are between the 2000-8 spectra presented here, while Conti \& Howarth (1999) report no variability between 1990-2002 in the $\sim 1 \mu \mathrm{m}$ region. Selected spectra between 1992-2008 are plotted in Fig. A.1. 
Table A.5. Summary of historical spectral observations of $\zeta^{1}$ Sco.

\begin{tabular}{|c|c|c|c|c|}
\hline Date & Wavelength & $\begin{array}{l}\text { Spectral } \\
\text { type }\end{array}$ & Comments & Reference \\
\hline$<1896$ & - & - & $H \beta$ in emission & Pickering (1896) \\
\hline $1891-1899$ & $4000-4900 \AA$ & B1p & $\begin{array}{c}\text { P Cygni profiles in } H \beta, \gamma \\
\text { all other lines in absorption } \\
\left(\mathrm{He}_{\mathrm{I}} 4009, \mathrm{O}_{\mathrm{II}} 4349, \mathrm{C}_{\mathrm{II}} 4267\right)\end{array}$ & Cannon (1901) \\
\hline 1924 & $4100-4900 \AA$ & $B(2) e q$ & $\begin{array}{c}\text { P Cygni profiles in } H \beta, \gamma \\
\text { H } \delta \text { in absorption }\end{array}$ & Merrill et al. (1925) \\
\hline $1903-1929$ & - & Ble Ia & - & Bidelman (1954) \\
\hline $1926-1930$ & $3950-4900 \AA$ & Blpe & - & Rimmer (1930) \\
\hline $30 / 07 / 34$ & - & - & P Cygni profiles in $\mathrm{H} \alpha$ and $\mathrm{D} 3 \mathrm{He} \mathrm{I}$ & Merril \& Burwell (1943) \\
\hline$<1938$ & - & - & Listed as variable & $\begin{array}{c}\text { Payne-Gaposchkin \& } \\
\text { Gaposchkin (1938) }\end{array}$ \\
\hline 1954-1958 & $3800-6600 \AA$ & $\mathrm{B} 1.5 \mathrm{Ia}^{+}$ & $\begin{array}{l}\text { No appreciable difference } \\
\text { from Cannon (1901) }\end{array}$ & Code \& Houck (1958) \\
\hline $26 / 04 / 58$ to $03 / 09 / 60^{1}$ & $3900-4500 \AA$ & Ble Ia & & Buscombe et al. (1962) \\
\hline $22 / 08 / 61$ & $6200-6600 \AA$ & - & Ho in emission & Jaschek et al. (1964) \\
\hline $7 / 08 / 66$ & $3800-4700 \AA$ & B1 Iae & $\begin{array}{l}H \beta \text { in emission, } H \gamma, \delta \\
\mathcal{G} H e \text { I } 4471 \text { in absn. }\end{array}$ & $\begin{array}{l}\text { Buscombe \& Kennedy } \\
\text { (1968) }\end{array}$ \\
\hline $\begin{array}{l}6 / 07 / 60 \\
9 / 08 / 60 \\
10 / 08 / 60 \\
28 / 06 / 66 \\
27 / 07 / 66\end{array}$ & $3100-6750 \AA$ & B1 Ia & $\begin{array}{c}\text { P Cygni profiles in } \\
\mathrm{H} \alpha, \beta \mathcal{E} \mathrm{He} \lambda \lambda 5876,6678 \\
\text { Remaining lines in absorption } \\
\text { Variability in } \mathrm{H} \alpha \text { profile, but } \\
\text { no global spectral evolution }\end{array}$ & Hutchings (1968) \\
\hline$<1969$ & blue end & B1.5Ia & - & Schild et al. (1969) \\
\hline $\begin{array}{l}21 / 04 / 65,20 / 05 / 67 \\
18 / 03 / 68,14 / 08 / 70\end{array}$ & $3500-4900 \AA$ & $\mathrm{B} 1 \mathrm{Ia}^{+}$ & $\begin{array}{l}\text { P Cygni profiles in } \mathrm{H} \beta, \gamma \\
\text { Variable } \mathrm{He}_{\mathrm{I}}, \mathrm{N}_{\text {II }} \& \mathrm{O}_{\text {II }} \text { phot. lines }\end{array}$ & $\begin{array}{l}\text { Jaschek \& Jaschek } \\
\text { (1973) }\end{array}$ \\
\hline 6/08/68 & $5800-6700 \AA$ & - & $\begin{array}{c}\text { P Cygni profiles in } \\
\mathrm{H} \alpha, \operatorname{He}\lrcorner \lambda \lambda 5876,6678\end{array}$ & Rosendhal (1973) \\
\hline $16 / 05 / 73$ to $10 / 07 / 73$ & blue end & - & No apparent variability in spec. type & Hutchings et al. (1976) \\
\hline $\begin{array}{l}28 / 04 / 74 \text { to } 1 / 05 / 74 \\
28 / 04 / 75\end{array}$ & $3800-4400 \AA$ & $B 1.5 \mathrm{Ia}^{+}$ & $\begin{array}{l}\text { No variability above } 20 \% \text { in } \\
\text { line strength reported }\end{array}$ & Walborn (1975) \\
\hline 1974 Oct. \& 1975 Mar. & $\sim \mathrm{H} \alpha$ & - & Variation in $\mathrm{H} \alpha$ P Cygni profile & Dachs et al. (1977) \\
\hline $26 / 06 / 72$ to $14 / 08 / 75^{2}$ & $3400-6700 \AA$ & B1Ia & $\begin{array}{c}\text { Variable P Cygni profiles in } \\
\mathrm{H} \alpha, \beta \text { \& } \mathrm{He} \mathrm{I} \lambda \lambda 5876,6678 \\
\text { No secular evolution in spec. type }\end{array}$ & $\begin{array}{l}\text { Sterken \& Wolf } \\
\quad(1978)\end{array}$ \\
\hline$<1984$ & $\begin{array}{l}4300-4900 \AA \\
5500-6800 \AA\end{array}$ & $\mathrm{B} 1.5 \mathrm{Ia}^{+}$ & $\begin{array}{l}\text { Variable P Cygni profiles in } \\
\qquad \mathrm{H} \alpha, \beta \& \mathrm{He}_{\mathrm{I}} \lambda 6678\end{array}$ & $\begin{array}{l}\text { Lopez \& Walsh } \\
\quad(1984)\end{array}$ \\
\hline $\begin{array}{l}1995^{4} \\
1990 \text { to } 1994^{3}\end{array}$ & $\begin{array}{l}3450-8630 \AA \\
4000-6740 \AA\end{array}$ & $\mathrm{B} 1.5 \mathrm{Ia}^{+}$ & $\begin{array}{c}\text { Variable P Cygni profiles in } \\
\mathrm{H} \alpha, \beta, \mathrm{He} \mathrm{I} \lambda 6678 \& \mathrm{Fe} \text { III } \\
\mathrm{H} \gamma, \delta \text { in absorption } \\
\text { No changes in He I:Si III ratio } \\
\& \text { hence spec. type }\end{array}$ & Rivinius et al. (1997) \\
\hline 1998 Oct. 7 & 4000-8950 ̊ & B1.5 $\mathrm{Ia}^{+}$ & $\begin{array}{l}\mathrm{H} \alpha, \beta, \mathrm{He}_{\mathrm{I}} \lambda 6678 \mathrm{P} \text { Cygni, higher } \\
\quad \text { Balmer series in absorption }\end{array}$ & This work \\
\hline 1999 June-July $^{5}$ & 4000-8950 ̊ & B1.5 $\mathrm{Ia}^{+}$ & $\begin{array}{c}\mathrm{H} \alpha, \beta, \text { Не }_{\perp} \lambda 6678 \mathrm{P} \text { Cygni, higher } \\
\text { Balmer series in absorption }\end{array}$ & This work \\
\hline 2002 March-May $^{6}$ & $5810-7205 \AA$ & - & Variable H $\alpha$ P Cygni & Morel et al. (2004) \\
\hline 2003 May-June & $4000-7000 \AA$ & $\mathrm{B} 1.5 \mathrm{Ia}^{+}$ & - & Crowther et al. (2006) \\
\hline 2005 April 24 & $3800-6800 \AA$ & $\mathrm{B} 1.5 \mathrm{Ia}^{+}$ & Н $\alpha, \beta \& \mathrm{He}_{\perp} \lambda 6678$ P Cygni, higher & This work \\
\hline
\end{tabular}


J. S. Clark et al.: On the nature of the galactic early-B hypergiants

Table A.5. continued.

\begin{tabular}{|c|c|c|c|c|}
\hline Date & Wavelength & $\begin{array}{l}\text { Spectral } \\
\text { type }\end{array}$ & Comments & Reference \\
\hline & \multicolumn{4}{|c|}{ Balmer series in absorption } \\
\hline 2006 Feb. 16 & 3933-7985 Å & B1.5 $\mathrm{Ia}^{+}$ & $\begin{array}{l}\mathrm{H} \alpha, \beta \text { \& He }{ }_{\mathrm{I}} \lambda 6678 \mathrm{P} \text { Cygni, higher } \\
\text { Balmer series in absorption }\end{array}$ & This work \\
\hline 2009 March 06 & $3060-5600 \AA$ & B1.5 $\mathrm{Ia}^{+}$ & $\begin{array}{c}\mathrm{H} \beta \text { P Cygni, higher Balmer } \\
\text { series in absorption }\end{array}$ & This work \\
\hline
\end{tabular}

Notes. A parameter is listed in italics if not explicitly given (wavelength of observation) or the data from which it is derived was not presented. Selected spectra between 1992-2009 are plotted in Fig. A.1. ${ }^{(1)} 6$ observations in this period, ${ }^{(2)} 44$ observations in this period, ${ }^{(3)} 233$ observations in this period, ${ }^{(4)} 57$ observations in this period, ${ }^{(5)} 14$ observations in this period. ${ }^{(6)} 6$ observations in this period. 
Table A.6. Summary of the dates of historic spectral classifications of the Galactic BHGs.

\begin{tabular}{lc}
\hline \hline Publication & $\begin{array}{c}\text { Observation } \\
\text { date }\end{array}$ \\
\hline \multicolumn{1}{c}{ HD 80077 (B2.5 Ia } \\
Morgan et al. (1955) & $1942-1955^{a}$ \\
Buscombe \& Kennedy (1968) & $1963-1967^{b}$ \\
Nordstrom (1975) & $<1975^{a}$ \\
Moffat \& Fitzgerald (1977) & $<1977^{a}$ \\
Knoechel \& Moffat (1982) & $1977^{a, b}$ \\
Negueruela et al. (in prep.) & 2008
\end{tabular}

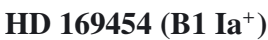

Morgan et al. (1955)

Hiltner (1956)

Code \& Houck (1958)

Botto \& Hack (1962)

Hutchings (1970)

Wolf \& Stahl (1985)

Walborn (1980)

Hutchings (1976)

Rivinius et al. (1997)

Hanson et al. (1996)

Hanson et al. (1998)

Groh et al. (2007)

HD $190603\left(\mathrm{~B} 1.5^{\mathrm{Ia}^{+}}\right)$

\begin{tabular}{lc} 
Morgan et al. (1955) & $1942-1955$ \\
Ahmad (1952) & $<1952$ \\
Hiltner (1956) & $<1956$ \\
Slettebak (1956) & $<1956$ \\
Lesh (1968) & $<1968$ \\
Hutchings (1970) & 1968 \\
Walborn (1971) & 1969 \\
Hutchings (1976) & $1973-1975$ \\
Bisiacchi et al. (1976) & $1975-1976$ \\
Andrillat et al. (1995) & $1988-1993$ \\
Rivinius et al. (1997) & $1990-1199^{b}$ \\
Lennon et al. (1992) & 1990 \\
Hanson et al. (1996) & $1994^{c, d}$ \\
Blum et al. (1997) & $1996^{c, d}$ \\
Hanson et al. (1998) & $1998^{c}$ \\
Crowther et al. (2006) & $1992-2003$ \\
Markova \& Puls (2008) & $<2007$ \\
& \\
Vidal (1973) & $1973^{d}$ \\
Bord (1979) & 1974 \\
Hammerschlag-Hensberge et al. (1979) & 1975 \\
& 1977 \\
Parkes et al. (1980) & 1978 \\
Kaper et al. (1995) & $1977-1978^{d}$ \\
Kaper et al. (2006) & 1984 \\
& $1996^{b}$ \\
\hline
\end{tabular}

Notes. All classifications made in the optical(4-6000 ̊) band unless otherwise noted $-{ }^{(a)}$ marginally earlier spectral type of B2 Iae given. (b) multiple spectra in this period. ${ }^{(c)}$ observations within the near-IR $(\sim 1-2.2 \mu \mathrm{m})$ window. ${ }^{(d)}$ marginally later spectral type of B1.5 or B2 Iae given.

\section{Appendix B: Spectropolarimetry}

Considering a simple "core-halo" wind model for heuristic purposes, electron scattering of photospheric radiation in an asymmetric outflow will generate a grey intrinsic linear polarization, while emission lines formed in the wind will see a smaller scattering optical depth, and so are expected to be less polarized (e.g., McLean 1979). This will result in depolarization through the line, although the addition of an interstellar-polarization vector means that this "line effect" often manifests in other ways, including an observed increase in degree of polarization, $P$.

Previously unpublished spectropolarimetric observations of Cyg OB2 \#12 and $\zeta^{1}$ Sco were obtained as part of the investigations reported by Harries et al. (2002). Cyg OB2 \#12 was observed on 1995 July 17 (using the WHT with ISIS spectrograph; $R \simeq 3300$ ), and $\zeta^{1}$ Sco on 1997 Jun. 5 (AAT with RGO spectrograph; $R \simeq 5000$ ), with the data acquisition and reduction essentially as in the manner described by Harries \& Howarth (1996). Results are displayed in Fig. B.1.

For Cyg OB2 \#12 there is no compelling evidence of a change in polarization through the $\mathrm{H} \alpha$ emission line (although there is a hint of a possible increase in $P$ ), and hence no strong indication of large-scale asymmetry in the electron-scattering envelope. Similarly, there is no strong evidence for temporal polarimetric variability; although the spread in published $R$-band photopolarimetric measurements is somewhat larger than their quoted formal errors, the results presented by Schmidt et al. (1992; $\left.P=7.893 \pm 0.037 \%, \theta=116.23 \pm 0.14^{\circ}\right)$ Whittet et al. $\left(1992 ; 7.97 \pm 0.05 \%, 117^{\circ}\right)$, and Kobulnicky et al. (1994; $\left.8.35 \pm 0.21 \%, 117.8^{\circ}\right)$ are all broadly consistent with the present results, as are earlier O-band measurements reported by Kruszewski (1971) and by McMillan \& Tapia (1977). We note, however, an anomalous result reported by Schulz \& Lenzen (1983; $\left.P=10.05 \pm 0.12 \%, \theta=125^{\circ}\right)$; their observations of two further stars agree well with measurements presented by Schmidt et al. (1992).

In spite of its brightness, $\zeta^{1}$ Sco has been much less extensively observed, with the only available point of direct comparison being the narrow-band $(\Delta \lambda=8.5 \AA) \mathrm{H} \alpha$ polarimetric measurement of $P=2.39 \pm 0.03 \%, \theta=64.6^{\circ}$ reported by McLean $\&$ Clarke $(1979)^{11}$. This is in agreement with our results; however, our spectropolarimetry clearly reveals a significant, $\sim 1.5^{\circ}$ position-angle rotation through the emission line.

Physically, the line effect may be associated with large-scale, axisymmetric structures (such as might result from rapid rotation), or irregular "clumps". For a star with parameters similar to those of $\zeta^{1}$ Sco, summarized in Table 4, the critical equatorial rotation velocity ${ }^{12}$ is $v_{\mathrm{e}} \simeq 210 \mathrm{~km} \mathrm{~s}^{-1}$. With a measured $v_{\mathrm{e}} \sin i$ of $\sim 45 \mathrm{~km} \mathrm{~s}^{-1}$ (Table 3 ), the intrinsic rotation is probably substantially subcritical $\left(v_{\mathrm{e}} / v_{\text {crit }} \lesssim 0.69\right.$ with $95 \%$ confidence). A rotationally-induced axisymmetric departure from spherical symmetry (i.e. a "disc" or "polar" wind) therefore seems a priori improbable as the origin of the line effect in this star. A more likely cause of the observed change in polarization is the presence of transient large-scale inhomogenities, or "clumps", as has been proposed for P Cygni and AG Car on the basis of spectropolarimetric observations by e.g. Nordsieck et al. (2001) and Davies et al. (2005); indeed CMFGEN model atmosphere analysis of both these stars confirms the presence of the significant wind clumping (Najarro et al. 2001; Groh et al. 2009a).

\footnotetext{
11 Matthewson \& Ford (1970) report a blue-band measurement of $P=$ $2.29 \%, \theta=67.3^{\circ}$.

12 The velocity at which the outward centrifugal force equals the inward gravitational force.
} 

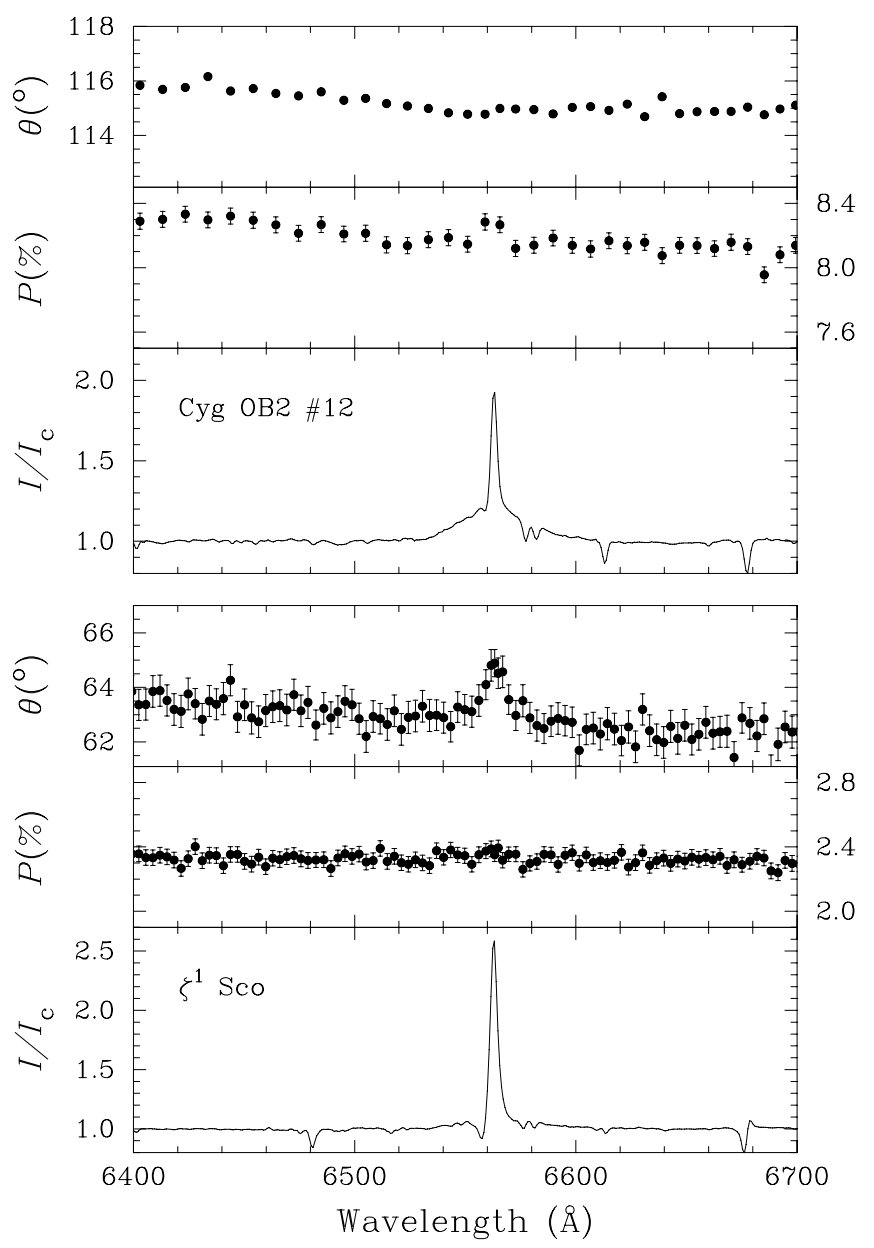

Fig. B.1. Linear spectropolarimetry of Cyg OB2 \#12 and $\zeta^{1}$ Sco. Rectified spectra are shown at full resolution; polarimetry is binned to an (almost) constant error of $0.05 \%$ in degree of polarization.

Therefore, by direct analogy, an additional observational test of this hypothesis for $\zeta^{1}$ Sco would be the detection of timedependent variations in polarization (under the assumption that there is no prefered geometry for the clumping), noting that spectroscopic observations are already strongly indicative of the presence of transient wind structure (Appendix A).

\section{Appendix C: The age of NGC 6231 and Sco OB1}

Given its proximity ( $1.64 \mathrm{kpc}$; Sana et al. 2008), NGC 6231, its host association Sco OB1, and the massive stars located within both have been the subject of numerous multiwavelength studies. Here we review these data in order to constrain the evolutionary history of both cluster and association to better understand the nature of $\zeta^{1}$ Sco. Photometric studies by Baume et al. (1999) suggest a significant age spread within NGC 6231, with star formation apparently commencing $\sim 10 \mathrm{Myr}$ ago, culminating in the formation of the massive stellar cohort 3-5 Myr ago. The latter age is consistent with the findings of Sana et al. (2006a, 2007), who report a similar value of 2-4 Myr from an analysis of the photometric data of (X-ray selected) pre-MS stars. An analagous study of the wider Sco OB1 association by Perry et al. (1991) supports a non coeval star formation history for this region, yielding an age of $\sim 8_{-3}^{+4.5} \mathrm{Myr}$.
Catalogues of the OB stellar content of both NGC 6231 and Sco OB1 are provided by Sana et al. (2006b) and Ankay et al. (2001) respectively, and allow for the individual placement of stars on the HR diagram presented in Fig. 13. For those stars in Sana et al. we adopted the luminosities given by these authors and utilised the spectral type/temperature relation of Martins et al. (2005). The temperatures and luminosities of the stars in Ankay et al. were again determined via the callibrations of Martins et al., with reddening for individual stars calculated via the intrinsic colours of Martins \& Plez (2006). Finally, given their rather evolved nature, we adopted the results of the tailored non-LTE analysis of Crowther \& Evans (2009) for HD 151804 and 152408 .

Comparison of these data to non-rotating and rotating Geneva isochrones clearly indicate that the region as a whole appears non coeval, at first glance being consistent with a spread of ages of between 2.5-5 Myr. Such a conclusion is supported by inspection of the properties of individual stars in both association and cluster, although interpretation is complicated by possible contamination of the latter by the former if, as seems likely, Sco OB1 hosts a younger population than is found in NGC 6231.

A large number of Main Sequence (MS) stars are found within NGC 6231, with the O8 V companion in the binary HD 152234 apparently being the earliest and defining the MS turnoff; consistent with an age of 5 Myr. While an O6 V companion to the WC7 star WR 79 has been reported (Hill et al. 2000) the 126 day binary period of HD 152234 will have ensured that neither component in this system will have interacted, while the 8.89 day period of WR79 indicates that significant mass transfer to the secondary may have occured (e.g. Petrovic et al. 2005). Encouragingly, the O9.7Ia primary of HD 152234 also lies on the $5 \mathrm{Myr}$ isochrone for rapid initial rotation, with the current $v \sin i$ also being consistent with this placement (Fraser et al. 2010).

Building on this approach, we find that HD 152219 (O9.5 III + B1-2 III-V) and HD 152134 (B0.5 Ia) lie upon the 5 Myr evolutionary track for non-rotating stars, with - sequentially - HD 326331 (O8 III((f))), HD 152247 (O9 III), HD 152249 (O9 Ib((f))) and HD 152234 (O9.7 Ia + O8 V) following the rotating track. In both cases the systematic progression to later spectral types with increasing luminosity class suggest these are real evolutionary sequences (cf. Cyg OB2; Negueruela et al. 2008).

However, there are a number of stars within NGC 6231 and Sco OB1 that appear incompatable with a $\sim 5 \mathrm{Myr}$ population: the O8 Iafpe/WN9ha stars HD 151804 and 152048 and the O5.5-7.5 III stars HD 151515, 152723,152233 and 152248. Regarding the former pair, Bohannan \& Crowther (1999) suggest a close physical kinship between these objects, apparently precluding an evolution through the cooler late-O/early-BSG phase present in NGC 6231. Of the mid-O giants, HD 151515 and 152723 appear somewhat subluminous for their spectral type but the properties of the O5.5 III(f) + O7.5 and O7.5 III(f) + O7 III(f) binaries HD 152233 and 152248 (Sana et al. 2008; 2001) clearly support the assertion that a younger population is present; although the dymanical masses for both components of the latter system appear somewhat lower than expected $\left(29.6 M_{\odot}\right.$ and $29.9 M_{\odot}$ respectively).

We therefore conclude that comparison of NGC 6231 to Sco OB1 implies that the respective stellar populations are non coeval. However, with the exception of HD 152233 and HD 152248, the massive stellar population of NGC6231 appears consistent with a single burst of star formation $\sim 5 \mathrm{Myr}$ ago. Indeed, no early-mid O MS stars consistent with a younger 
population appears present within NGC 6231, while the population of late-O/early-B MS stars that are present are systematically displaced redwards from the $\leq 3$ Myr isochrones. Therefore, if they are members of NGC 6231, we cannot exclude the possibility that, with spectral types <O8, HD 152233 and HD 152248 are in fact bona fide blue stragglers; having evolved via a different pathway from the majority of stars and hence that NGC 6231 is truly coeval. In this respect it would therefore closely resemble $\mathrm{Cyg} \mathrm{OB} 2$, for which Negueruela et al. (2008) arrived at a comparable conclusion.

\section{Appendix D: Spectral fits to $\zeta^{1}$ Sco and HD 190603}

We present additional model fits to $\zeta^{1}$ Sco based on the FEROS (3700-8850 $\AA$ ) optical data (not presented in the paper version due to reasons of space). 
J. S. Clark et al.: On the nature of the galactic early-B hypergiants
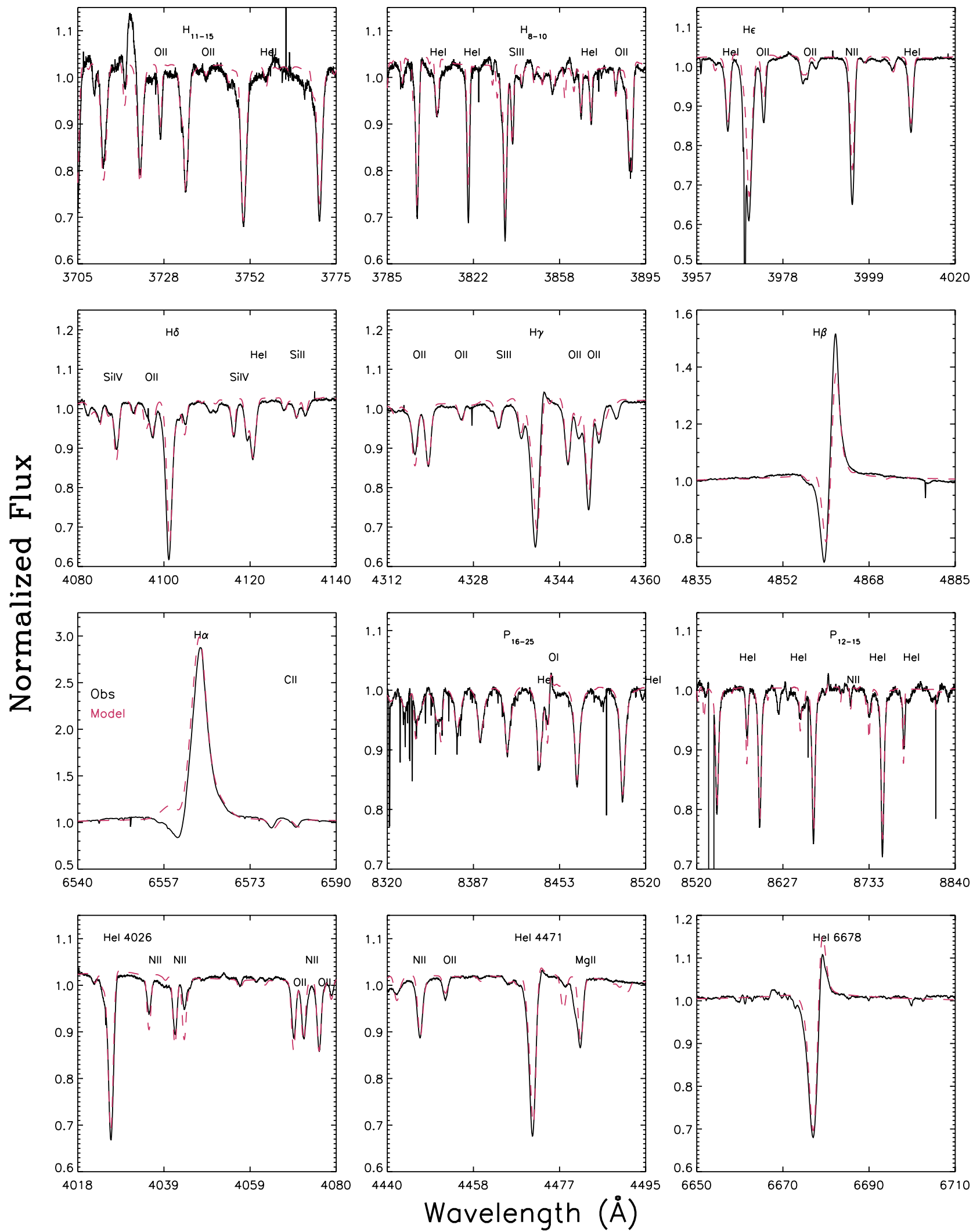

Fig. D.1. Comparison of the synthetic spectrum of $\zeta^{1}$ Sco (red dashed line) to observational data for various transitions between $4090-22100 \AA$. Optical data correspond to the FEROS data kindly provided by Otmar Stahl. Note the lack of long-term variability between these data and those presented in Fig. 7. 

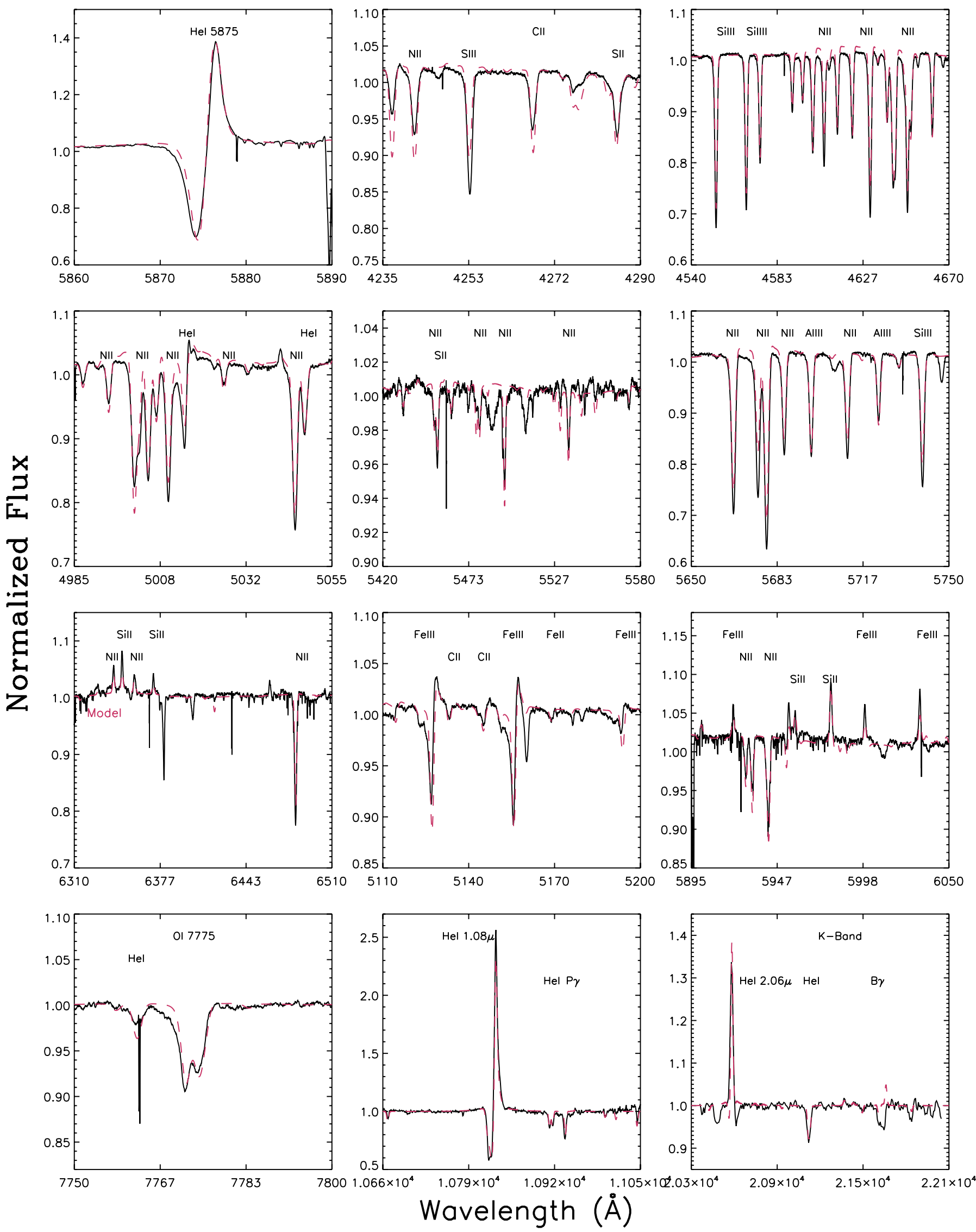

Fig. D.1. continued. 